SERVIÇO DE PÓS-GRADUAÇÃO DO ICMC-USP

Data de Depósito: 8 de abril de 2010

Assinatura:

\title{
Atratores para uma classe de equações de vigas extensiveis fracamente dissipativas
}

\author{
Vando Narciso \\ Orientador: Prof. Dr. Ma To Fu \\ Tese apresentada ao Instituto de Ciências Matemáticas e de \\ Computação - ICMC-USP, como parte dos requisitos para \\ obtenção do título de Doutor em Ciências - Matemática.
}

\section{USP - São Carlos}

Abril/2010 
A minha esposa Léia, as minhas filhas

Emanuele e Gabriela, e aos meus pais Orlando e Guiomar (in memorian). 


\section{Agradecimentos}

Primeiramente agradeço a Deus pela oportunidade que me concedeu para concluir mais esta etapa da minha vida.

À minha esposa Léia que confiou em mim e sempre esteve ao meu lado.

Às minhas filhas Emanuele e Gabriela.

Aos meus pais Orlando e Guiomar (in memorian).

Ao prof. Dr. Ma To Fu, meu orientador e amigo, por seus sábios conselhos e orientação.

Aos professores do ICMC - USP que contribuiram para o meu crescimento profissional.

Finalmente, agradeço à UEMS e à FUNDECT pelo apoio financeiro para realização deste trabalho. 


\section{Resumo}

Este trabalho contém resultados sobre a existência, unicidade e comportamento assintótico de soluções para uma equação de viga não linear do tipo Kirchhoff,

$$
u_{t t}+\Delta^{2} u-M\left(\int_{\Omega}|\nabla u|^{2} d x\right) \Delta u+f\left(u_{t}\right)+g(u)=h \quad \text { em } \quad \Omega \times \mathbb{R}^{+},
$$

onde $\Omega \subset \mathbb{R}^{N}$ é um domínio limitado com fronteira regular $\Gamma$. Essa equação é um modelo matemático para pequenas vibrações transversais de vigas ou placas extensíveis. O termo não local $M\left(\int_{\Omega}|\nabla u|^{2} d x\right) \Delta u$ está relacionado à variação de tensão na viga devida à sua extensibilidade. O termo $f\left(u_{t}\right)$ representa uma dissipação para o sistema e $g(u)$ representa a força exercida pelo meio. A função $h$ representa uma força externa adicional. Consideramos o problema com as condições de fronteira $\left.u\right|_{\Gamma \times \mathbb{R}^{+}}=\left.\frac{\partial u}{\partial v}\right|_{\Gamma \times \mathbb{R}^{+}}=0$, que corresponde ao modelo de vigas fixadas pelo bordo $\Gamma$. Discutiremos o caso em que a dissipação é linear e o caso em que é não linear. Mostraremos que em ambos os casos o sistema dinâmico associado ao problema possui um atrator global. Entretanto, para o caso em que a dissipação é linear, obtemos num espaço de fase mais regular, a existência de um conjunto inércia de dimensão finita, que atrai exponencialmente todos os limitados deste espaço. 


\begin{abstract}
This work contains some results on the existence, uniqueness and asymptotic behavior of solutions for a nonlinear beam equation of Kirchhoff type,

$$
u_{t t}+\Delta^{2} u+M\left(\int_{\Omega}|\nabla u|^{2} d x\right) \Delta u+g\left(u_{t}\right)+f(u)=h
$$

where $\Omega \subset \mathbb{R}^{N}$ is a bounded domain with smooth boundary $\Gamma$. This equation is a model for small vibrations of extensible beams. The nonlocal term $M\left(\int_{\Omega}|\nabla u|^{2} d x\right) \Delta u$ is related to the variation of tensions in the beam due to its extensibility. The term $f\left(u_{t}\right)$ represents a damping mechanism for the system and $g(u)$ represents the force exerted by the foundation. The function $h$ represents an additional external force. We consider the problem with boundary condition $\left.u\right|_{\Gamma \times \mathbb{R}^{+}}=\left.\frac{\partial u}{\partial v}\right|_{\Gamma \times \mathbb{R}^{+}}=0$, which corresponds to the model of clamped beams. We discuss the cases where the dissipation is linear and the case nonlinear. We show that in both cases, the dynamical system associated to the problem has a global attractor. However, when the dissipation is linear, we obtain, in a more regular space, the existence of an inertial set of finite dimension, which attracts exponentially all bounded sets of this space.
\end{abstract}




\section{Índice}

Introdução $\quad$ xii

1 Preliminares 5

$1.1 \quad$ Espaços de Sobolev . . . . . . . . . . . . . . . . . . . . . 5

1.1.1 Espaços de Sobolev $W^{m, p}(\Omega) \quad \ldots \ldots \ldots \ldots \ldots$

1.1.2 Espaços $L^{p}((a, b), X) \ldots \ldots \ldots \ldots \ldots$

1.1.3 Espaços de interpolação .................... 9

1.1.4 Desigualdades elementares . . . . . . . . . . . . . . . 10

1.2 Aspectos Básicos da Teoria de Semigrupos . . . . . . . . . . . . . . . . . 12

1.2.1 Semigrupos de operadores lineares de classe $C_{0} \ldots \ldots \ldots 12$

1.2.2 Teoremas de Hille-Yosida e de Lumer-Phillips . . . . . . . . . . . . . 14

1.2.3 Problema de valor inicial não homogêneo . . . . . . . . . . . . . . 15

1.3 Atrator Global e Atrator Exponencial . . . . . . . . . . . . . . . . . . . 17

1.3.1 Definição de atrator global . . . . . . . . . . . . . . . 17

1.3.2 Dimensão fractal e dimensão de Hausdorff ～. . . . . . . . . . . . . . 20

1.3.3 Atrator exponencial (conjunto inércia) . . . . . . . . . . . . . . 21

2 Solução Global para o Problema com Dissipação Linear 23

2.1 Existência de Solução Global . . . . . . . . . . . . . . . . . . . . . . . . . 24

2.1.1 Existência de solução forte . . . . . . . . . . . . . . . . . . . . . 24

2.1.2 Existência de solução fraca . . . . . . . . . . . . . . . . . . . . 34 
3 Atrator Exponencial para o Problema com Dissipação Linear 37

3.1 Existência de um Conjunto Absorvente em $E_{0} \ldots \ldots \ldots$. . . . . . . 38

3.2 Atrator Global em $E_{0} \ldots \ldots \ldots \ldots \ldots$. . . . . . . . . . . . 42

3.3 Existência de um Conjunto Absorvente em $E_{1} \ldots \ldots \ldots$. . . . . . . . . . . 47

3.4 Atrator Exponencial (Conjunto Inércia) ～. . . . . . . . . . . . . . . 51

4 Atrator Global para o Problema com Dissipação Não Linear 59

4.1 Existência de Solução Global . . . . . . . . . . . . . . . . . . . . . 61

4.2 Existência de Atrator Global . . . . . . . . . . . . . . . . . . . . 63

4.2.1 Conjunto absorvente em $E_{0} \ldots \ldots \ldots \ldots$. . . . . . . . . 64

4.2 .2 Compacidade assintótica em $E_{0} \ldots \ldots \ldots$. . . . . . . . . . 68

$\begin{array}{ll}\text { Referências Bibliográficas } & 75\end{array}$ 


\section{Introdução}

Seja $\Omega \subset \mathbb{R}^{N}$ um conjunto aberto limitado com fronteira suave $\Gamma=\partial \Omega$. Neste trabalho investigaremos a existência, unicidade e comportamento assintótico de soluções do modelo não linear para vibrações de vigas do tipo Berger-Kirchhoff

$$
u_{t t}+\Delta^{2} u-M\left(\int_{\Omega}|\nabla u|^{2} d x\right) \Delta u+f\left(u_{t}\right)+g(u)=h \quad \text { em } \quad \Omega \times \mathbb{R}^{+},
$$

satisfazendo as condições iniciais

$$
\left.u\right|_{t=0}=u_{0} \quad \text { e }\left.\quad u_{t}\right|_{t=0}=u_{1} \quad \text { em } \quad \Omega,
$$

e condições de fronteira

$$
\left.u\right|_{\Gamma \times \mathbb{R}^{+}}=\left.\frac{\partial u}{\partial v}\right|_{\Gamma \times \mathbb{R}^{+}}=0 .
$$

O termo não local $M\left(\int_{\Omega}|\nabla u|^{2} d x\right) \Delta u$ está relacionado à variação de tensão na viga devida à sua extensibilidade. O termo $f\left(u_{t}\right)$ representa uma dissipação para o sistema e $g(u)$ representa a força exercida pelo meio. A função $h$ representa uma força externa adicional. As condições de fronteira acima modelam o caso em que a viga é fixada pelo bordo $\Gamma$.

A equação (0.1) é uma generalização do modelo introduzido por Woinowsky-Krieger,

$$
\frac{\partial^{2} u}{\partial t^{2}}+\frac{E I}{\rho} \frac{\partial^{4} u}{\partial x^{4}}-\left(\frac{H}{\rho}+\frac{E A}{2 \rho L} \int_{0}^{L}\left|\frac{\partial u}{\partial x}\right|^{2} d x\right) \frac{\partial^{2} u}{\partial x^{2}}=h
$$


que descreve as vibrações transversais de uma viga extensível, de comprimento $L$, fixa nas extremidades e sujeita a uma força externa $h$. A quantidade $H$ representa uma força axial, $E$ o módulo de Young, $I$ a seção transversal do momento de inércia, $\rho$ a densidade de massa, e $A$ a seção transversal de área. Aspectos de modelagem deste problema também foram discutidos e extendidos por Berger [8], Eisley [23] e mais recentemente, por Arosio [1].

No caso em que o termo de quarta ordem não aparece em $(0.1)$ ou (0.4), obtemos a equação da onda de Kirchhoff [26], que foi bem estudada por vários autores. Veja por exemplo, [2, 13, $17,30,32,37,44]$ e suas referências.

Voltando ao problema (0.1), os primeiros resultados de análise matemática rigorosa apareceram já nos anos 1970. Podemos citar, por exemplo, os artigos de Ball $[4,5]$, onde são considerados a existência global, via método de Galerkin, e a estabilidade assintótica na presença de termos de dissipação (dampings). Também é importante mencionarmos o trabalho de Dickey [19] que usa série Fourier. Para dimensões maiores, um dos primeiros resultados foi obtido por Medeiros [34], usando método de Galerkin e estabelecendo hipóteses apropriadas sobre a função $M$.

Mais tarde, vários resultados interessantes foram publicados focalizando as diversas propriedades qualitativas do problema. Por exemplo, dissipações fracas, dissipações localizadas, soluções com dados iniciais pequenos, problemas com termos de memória, sistemas de termoelasticidade, entre outros. Citamos, por exemplo, os trabalhos [10, 11, 15, 16, 27, 31, 36, 43] e suas referências.

Entretanto, na presença simultânea de três termos não lineares

$$
f\left(u_{t}\right)=\left|u_{t}\right|^{r} u_{t}, \quad g(u)=|u|^{\rho} u, \quad \text { e } \quad M\left(\|\nabla u\|_{2}^{2}\right)
$$

o problema da estabilidade assintótica foi considerado com sucesso, apenas recentemente, em Cavalcanti et al [14]. Discutiremos esse caso no Capítulo 4.

Nosso trabalho é dedicado à existência de atratores para o problema (0.1)-(0.3). A existência de atrator global para o modelo (0.4) foi primeiramente investigada por Hale [24]. Depois Eden and Milani [21] estudaram (0.1) no caso em que $g=0, f\left(u_{t}\right)=u_{t}, M$ linear, e com condições de fronteira $u=\Delta u=0$ sobre $\Gamma \times \mathbb{R}^{+}$. Quando $g=0$ e com um termo de dissipação forte $f\left(u_{t}\right)=(-\Delta)^{\beta} u_{t}$, com $0<\beta \leq 1$, Biazutti e Crippa [10] provaram a existência de um atrator exponencial para o problema (0.1). Alguns resultados relacionados podem ser encontrados em $[25,45]$. 
Neste trabalho propomos analisar o problema (0.1)-(0.3) sob dois aspectos. O primeiro, quando a dissipação $f\left(u_{t}\right)$ é linear, e o segundo, quando $f\left(u_{t}\right)$ é não linear. Os resultados obtidos foram divididos nos capítulos 2, 3 e 4, sendo os dois primeiros para o caso linear e o último para o caso não linear.

No Capítulo 2, primeiramente, obtemos a existência e unicidade de solução local forte para o problema (0.1)-(0.3) no espaço energia $E_{1}=H^{4}(\Omega) \cap H_{0}^{2}(\Omega) \times H_{0}^{2}(\Omega)$, via teoria de semigrupos e, em seguida, usando estimativas uniformes obtemos uma solução global em $E_{1}$. Finalizando o capítulo, através de argumentos de densidade, obtemos a existência e unicidade de solução global fraca no espaço energia $E_{0}=H_{0}^{2}(\Omega) \times L^{2}(\Omega)$.

No Capítulo 3, mostramos a existência de conjuntos absorventes para o problema (0.1) em $E_{0}$ e $E_{1}$, e a existência de um atrator global em $E_{0}$. Na última seção provamos a existência de um conjunto inércia para o sistema dinâmico associado ao problema (0.1)-(0.3), que atrai exponencialmente todos os conjuntos limitados de $E_{1}$ com respeito à norma de $E_{0}$, usando a teoria presente em Eden et al. [22]. O resultado principal do capítulo é o Teorema 3.4.1.

No caso do problema com dissipação não linear, Capítulo 4, enunciamos os resultados de existência e unicidade de solução, cujas provas podem ser deduzidas de Cavalcanti et al. [14]. Mostramos a existência de um conjunto absorvente e a existência de global atrator em $E_{0}$, explorando os mesmos argumentos do caso linear. Entretanto, um resultado que ainda fica em aberto é a prova da existência de um conjunto inércia para o problema (0.1) com dissipação não linear. O resultado principal do capítulo é o Teorema 4.2.1. 


\section{Capítulo}

\section{Preliminares}

\subsection{Espaços de Sobolev}

Nesta seção faremos uma breve recordação de alguns resultados importantes sobre os espaços de Sobolev que utilizaremos nos capítulos seguintes.

Seja $\Omega$ um subconjunto aberto e limitado do $\mathbb{R}^{N}$. Para $p \geq 1$, denotamos por $L^{p}(\Omega)$ a classe de todas as funções Lebesgue mensuráveis $u$, para as quais $|u|^{p}$ é uma função integrável sobre $\Omega$. $\operatorname{Em} L^{p}(\Omega)$ definimos a norma usual

$$
\|u\|_{p}=\left(\int_{\Omega}|u(x)|^{p} d x\right)^{\frac{1}{p}}, \quad 1 \leq p<\infty .
$$

$\operatorname{Assim}\left(L^{p}(\Omega),\|\cdot\|_{p}\right)$ é um espaço de Banach. No caso $p=\infty, L^{p}(\Omega)$ é o espaço formado por todas as funções $u$, essencialmente limitadas sobre $\Omega$. $\operatorname{Em} L^{\infty}(\Omega)$ definimos a norma

$$
\|u\|_{\infty}=\underset{x \in \Omega}{\operatorname{supess}}|u(x)|
$$

Também neste caso $L^{\infty}(\Omega)$ é um espaço de Banach. Quando $p=2, L^{2}(\Omega)$ é um espaço de 
Hilbert com produto interno e norma dados, respectivamente, por

$$
(u, v)=\int_{\Omega} u(x) v(x) d x \text { e }\|u\|_{2}=\left(\int_{\Omega}|u(x)|^{2} d x\right)^{\frac{1}{2}}
$$

Propriedades básicas sobre os espaços $L^{p}(\Omega)$ podem ser encontradas em, por exemplo, Brezis [12].

\subsubsection{Espaços de Sobolev $W^{m, p}(\Omega)$}

Dados um muiti-índice $\alpha=\left(\alpha_{1}, \alpha_{2}, \cdots, \alpha_{N}\right) \in \mathbb{N}^{N}$ e $z=\left(z_{1}, z_{2}, \cdots, z_{N}\right) \in \mathbb{R}^{N}$ define-se

$$
|\alpha|=\alpha_{1}+\alpha_{2}+\cdots+\alpha_{N}, \quad z^{\alpha}=z_{1}^{\alpha_{1}} z_{2}^{\alpha_{2}} \cdots z_{N}^{\alpha_{N}}, \quad \alpha !=\alpha_{1} ! \alpha_{2} ! \cdots \alpha_{N} !
$$

Denota-se por $D^{\alpha}$ o operador de derivação de ordem $\alpha$ definido por

$$
D^{\alpha}=\frac{\partial^{|\alpha|}}{\partial x_{1}^{\alpha_{1}} \partial x_{2}^{\alpha_{2}} \cdots \partial x_{N}^{\alpha_{N}}}, \quad|\alpha| \neq 0,
$$

e para $\alpha=(0,0, \cdots, 0)$ define-se $D^{0} u=u$ para toda função $u$. Por $D_{i}$ para $i=1,2, \cdots, N$ representa-se a derivação parcial $\frac{\partial}{\partial x_{i}}$.

Seja $\Omega$ um conjunto limitado ou ilimitado do $\mathbb{R}^{N}$ com fronteira suave $\Gamma$. Para $m \in \mathbb{N}, 1 \leq$ $p \leq \infty, W^{m, p}(\Omega)$ é definido como o espaço das funções $u$ em $L^{p}(\Omega)$ tais que para todo $|\alpha| \leq m$, as derivadas $D^{\alpha} u$ no sentido das distribuições, pertencem a $L^{p}$. Então, é bem conhecido que $W^{m, p}(\Omega)$ é um espaço de Banach com a norma

$$
\|u\|_{m, p}=\left(\sum_{|\alpha| \leq m}\left\|D^{\alpha} u\right\|_{p}^{p}\right)^{\frac{1}{p}}
$$

Quando $p=2$, usualmente denotamos $W^{m, p}(\Omega)$ por $H^{m}(\Omega)$ e este é um espaço de Hilbert com o correspondente produto interno.

Seja $C^{k}(\Omega), k \in \mathbb{N}$ ou $k=\infty$ o espaço das funções $k$ continuamente diferenciáveis sobre $\Omega$, e $C_{0}^{m}(\Omega)$ a totalidade das funções pertencentes a $C^{m}(\Omega)$ com suporte compacto em $\Omega$. O fecho de $C_{0}^{\infty}(\Omega)$ em $W^{m, p}(\Omega)$ é denotado por $W_{0}^{m, p}(\Omega)$ que é um subespaço de $W^{m, p}(\Omega)$. 
Teorema 1.1.1 (Teorema de Densidade) Se $\Omega$ é de classe $C^{m}, m \geq 1$, então $C^{m}(\bar{\Omega})$ é denso em $W^{m, p}(\Omega), 1 \leq p<\infty$.

Observação 1.1.1 $C^{m}(\bar{\Omega})$ modula a eleição de um representante contínuo.

Teorema 1.1.2 (Teorema de Imersão Compacta) Suponha que $\Omega$ é um aberto limitado de classe $C^{m}$. Então temos,

(i) Se $m p<N$, então $W^{m, p}(\Omega)$ esta imerso continuamente em $L^{q^{*}}(\Omega) \operatorname{com} \frac{1}{q^{*}}=\frac{1}{p}-\frac{m}{N}$ :

$$
W^{m, p}(\Omega) \hookrightarrow L^{q^{*}}(\Omega)
$$

Além disso, a imersão é compacta para qualquer $q, 1 \leq q<q^{*}$.

(ii) Se $m p=N$, então $W^{m, p}(\Omega)$ esta continuamente imerso em $L^{q}$, para todo $q, 1 \leq q<\infty$ :

$$
W^{m, p}(\Omega) \hookrightarrow L^{q}(\Omega)
$$

Além disso, a imersão é compacta, para todo $q, 1 \leq q<\infty$. Se $p=1, m=N$, então a imersão é válida para $q=\infty$.

(iii) Se $k+1>m-\frac{N}{p}>k, k \in \mathbb{N}$, então escrevendo $m-\frac{N}{p}=k+\alpha, k \in \mathbb{N}, 0<\alpha<1, W^{m, p}(\Omega)$ está continuamente imerso em $C^{k, \alpha}(\bar{\Omega})$ :

$$
W^{m, p}(\Omega) \hookrightarrow C^{k, \alpha}(\bar{\Omega}),
$$

onde $C^{k, \alpha}(\bar{\Omega})$ é o espaço das funções em $C^{k}(\bar{\Omega})$ cujas derivadas de ordem $k$ são Holder contínua com expoente $\alpha$. Além disso, se $N=m-k-1, e \alpha=1, p=1$, então a imersão também é válida para $\alpha=1$, e o operador imersão é compacto de $W^{m, p}(\Omega)$ em $C^{k, \beta}(\bar{\Omega})$, para todo $0 \leq \beta<\alpha$.

As propriedades de imersão $(i)$ - (iii) são válidas para domínios ilimitados ou $\mathbb{R}^{N}$ e mostram que $L^{q}(\Omega)$ em $(i i)$ e $C^{k, \alpha}(\Omega)$ em $(i i i)$ pode ser substituído por $L_{l o c}^{q}(\Omega)$ e $C^{k, \alpha}(\mathscr{B})$ para qualquer domínio $\mathscr{B} \subset \Omega$, respectivamente.

Seja $\Omega$ um aberto limitado de classe $C^{m}$ e $u \in W^{m, p}(\Omega)$. Então podemos definir o traço de $u$ sobre $\Gamma$ que coincide com os valores de $u$ sobre $\Gamma$ quando $u$ é uma função suave de $C^{m}(\bar{\Omega})$.

Teorema 1.1.3 (Teorema do Traço) Seja $v=\left(v_{1}, \cdots, v_{N}\right)$ o vetor normal unitário exterior sobre $\Gamma e$

$$
\gamma_{j} u=\left.\frac{\partial^{j} u}{\partial v^{j}}\right|_{\Gamma}, \quad \forall u \in C^{m}(\bar{\Omega}), \quad j=1, \cdots, m-1
$$


Então o operador traço $\gamma=\left\{\gamma_{0}, \cdots, \gamma_{m-1}\right\}$ pode ser estendido unicamente a um operador contínuo de $W^{m, p}(\Omega)$ em $\Pi_{j=1}^{m-1} W^{m-j-\frac{1}{p}, p}(\Gamma)$ :

$$
\begin{aligned}
\gamma: W^{m, p}(\Omega) & \rightarrow \Pi_{j=1}^{m-1} W^{m-j-\frac{1}{p}, p}(\Gamma) \\
u & \mapsto \gamma u=\left\{\gamma_{0} u, \cdots, \gamma_{m-1} u\right\}
\end{aligned}
$$

Além disso, é uma aplicação sobrejetiva.

Note que $W^{m-j-\frac{1}{p}, p}(\Gamma)$ são espaços com derivadas de ordem fracionária. Indicamos livro de Lions e Magenes [28] para definição e outras propriedades.

Seja $\Omega$ um aberto limitado do $\mathbb{R}^{N}$ com fronteira $\Gamma$ de classe $C^{1}$. Então para qualquer função $u \in H^{1}(\Omega)$, vale a seguinte estimativa

$$
\|u\|_{2_{\mid \Gamma}} \leq C\|u\|_{H^{1}(\Omega)}^{\frac{1}{2}}\|u\|_{2}^{\frac{1}{2}}
$$

onde $C$ é uma constante positiva independente de $u$.

\subsubsection{Espaços $L^{p}((a, b), X)$}

Seja $X$ um espaço de Banach e seja $-\infty \leq a<b \leq \infty$. Então $L^{p}((a, b) ; X), 1 \leq p<\infty$, denota o espaço de funções vetoriais $L^{p}$ de $(a, b)$ sobre $X$. Este espaço é um espaço de Banach munido da norma

$$
\|f\|_{L^{p}((a, b) ; X)}=\left(\int_{a}^{b}\|f(t)\|_{X}^{p} d t\right)^{\frac{1}{p}}
$$

onde o integral é entendido no sentido Bochner.

Para $p=\infty, L^{\infty}((a, b) ; X)$ é o espaço das funções mensuráveis de $(a, b)$ em $X$ essencialmente limitadas. É também um espaço de Banach com a norma

$$
\|f\|_{L^{\infty}((a, b) ; X)}=\operatorname{supess}_{t \in(a, b)}\|f(t)\|_{X} .
$$

Similarmente, quando $-\infty<a<b<\infty$, podemos definir o espaço de Banach $C^{k}([a, b] ; X)$ com a norma

$$
\|f\|_{C^{k}([a, b] ; X)}=\sum_{t=0}^{k} \max _{t \in[a, b]}\left\|\frac{d^{i} f}{d t^{i}}(t)\right\|_{X} .
$$




\subsubsection{Espaços de interpolação}

Relembremos alguns fatos sobre operadores lineares associados com uma forma bilinear e espaços de interpolação associados a um operador positivo definido em espaços de Hilbert.

Seja $V$ e $H$ espaços de Hilbert separáveis tal que $V$ é denso em $H$ e a imersão $V \hookrightarrow H$ é contínua e compacta. Assim, pelo Teorema de representação de Riesz podemos escrever

$$
V \subset H \equiv H^{\prime} \subset V^{\prime}
$$

Denotaremos o produto dual entre $V$ e $V^{\prime}$ por $\langle\cdot, \cdot\rangle$ e o produto interno em $H$ por $(\cdot, \cdot)$.

Seja $A$ um operador linear contínuo de $V$ em $V^{\prime}$. Podemos associá-lo com uma forma bilinear $a(.,$.$) sobre V$ de maneira que

$$
a(u, v)=\langle A u, v\rangle, \quad \forall u, v \in V .
$$

Supondo que $a$ é simétrico:

$$
a(u, v)=a(v, u)
$$

e também supondo que $a$ é coercivo, isto é, existe uma constante $\alpha>0$ tal que

$$
a(u, u) \geq \alpha\|u\|_{V}^{2}, \forall u \in V .
$$

Seja

$$
D(A)=\{u: u \in V, A u \in H\}
$$

Então pelo teorema de Lax-Milgran, $a(u, v)$ pode ser considerado um produto interno equivalente. Além disso, $A$ é um operador auto-adjunto estritamente positivo em $H$ e o teorema espectral nos permite definir as potências $A^{s}$ de $A$ para $s \in \mathbb{R}$. Como assumimos que a imersão $V \hookrightarrow H$ é compacta, existe (ver Yosida [50]) uma base ortonormal completa $\left\{w_{j}\right\}$ de $H$ e uma sequência $\left\{\lambda_{j}\right\}$ tal que $w_{j} \in D(A)$ e

$$
\left\{\begin{array}{l}
A w_{j}=\lambda_{j} w_{j}, \quad j=1,2, \cdots \\
0<\lambda_{1} \leq \lambda_{2} \leq \cdots, \lambda_{j} \rightarrow \infty, \text { com } j \rightarrow \infty \\
a\left(w_{i}, w_{j}\right)=\lambda_{i} \delta_{i j}, \quad \forall i, j \in \mathbb{N}
\end{array}\right.
$$


Assim, para $s>0$, definimos

$$
D\left(A^{s}\right)=\left\{u \in H ; \sum_{j=1}^{\infty} \lambda_{j}^{2 s}\left|\left(u, w_{j}\right)\right|^{2}<\infty\right\},
$$

$\mathrm{e}$

$$
\|u\|_{D\left(A^{s}\right)}=\left(\sum_{j=1}^{\infty} \lambda_{j}^{2 s}\left|\left(u, w_{j}\right)\right|^{2}\right)^{\frac{1}{2}} .
$$

Para $s$ negativo, $D\left(A^{s}\right)$ é o complemento de $H$ para a norma $\left(\sum_{j=1}^{\infty} \lambda_{j}^{2 s}\left|\left(u, w_{j}\right)\right|^{2}\right)^{\frac{1}{2}}$. Em particular, temos $V=D\left(A^{\frac{1}{2}}\right)$.

Sejam $X$ e $Y$ espaços de Banach, $\operatorname{com} X \subset Y, X$ denso em $Y$, e a imersão de $X \hookrightarrow Y$ contínua. Em geral existem várias maneiras de definir os espaços intermediários entre $X$ e $Y$. O que foi descrito acima dá uma espécie de definição de espaço intermediário, ou seja, os espaços de interpolação.

Seja $X:=V=D\left(A^{\frac{1}{2}}\right), Y:=H=D\left(A^{0}\right)$, e $a(u, v)=(u, v)_{X}$.

Então os espaços de interpolação $[X, Y]_{\theta}, 0 \leq \theta \leq 1$ são dados por

$$
[X, Y]_{\theta}=D\left(A^{\frac{1-\theta}{2}}\right), \forall \theta \in[0,1]
$$

e segue a seguinte desigualdade

$$
\|u\|_{[X, Y]_{\theta}} \leq C(\theta)\|u\|_{X}^{1-\theta}\|u\|_{Y}^{\theta}, \forall u \in X, \theta \in[0,1]
$$

Um exemplo típico do quadro acima é

$$
V=D\left(A^{1 / 2}\right)=H_{0}^{2}(\Omega), H=D\left(A^{0}\right)=L^{2}(\Omega), A=\Delta^{2} .
$$

\subsubsection{Desigualdades elementares}

A seguir apresentamos uma coleção de resultados elementares, mas fundamentais, sobre desigualdades que utilizamos com frequência nos capítulos seguintes. 
Teorema 1.1.4 (Desigualdade de Young) Seja $1<p, q<\infty \operatorname{com} \frac{1}{p}+\frac{1}{q}=1$. Então

$$
a b \leq \frac{a^{p}}{p}+\frac{b^{q}}{q}, \quad(a, b>0)
$$

Corolário 1.1.1 (Desigualdade de Young com $\varepsilon$ ) Seja $1<p, q<\infty \operatorname{com} \frac{1}{p}+\frac{1}{q}=1$. Então

$$
a b \leq \varepsilon a^{p}+C(\varepsilon) b^{q}, \quad(a, b>0)
$$

$\operatorname{para} C(\varepsilon)=(\varepsilon p)^{-q / p} q^{-1}$

Teorema 1.1.5 (Desigualdade de Poincaré) Seja $1 \leq p<\infty$. Então existe uma constante $C$ (dependendo da medida de $\Omega$ e de p) tal que

$$
\|u\|_{p} \leq C\|\nabla u\|_{p}, \quad \forall u \in W_{0}^{1, p}(\Omega)
$$

Lema 1.1.1 (Desigualdade de Gronwall). Sejam $\phi \in L^{\infty}\left(t_{0}, T\right)$ e $\beta \in L^{1}\left(t_{0}, T\right)$ tais que $\beta>0$, $\phi \geq 0$ e $\alpha \geq 0$ uma constante. Se

$$
\phi(t) \leq \alpha+\int_{t_{0}}^{t} \beta(s) \phi(s) d s, \text { para } t_{0} \leq t \leq T
$$

então

$$
\phi(t) \leq \alpha e^{\int_{t_{0}}^{t} \beta(s) d s}, t_{0} \leq t \leq T
$$

Lema 1.1.2 (Desigualdade de Gronwall Generalizada) Se $\phi, \alpha \in L^{\infty}\left(t_{0}, T\right)$ e $\beta \in L^{1}\left(t_{0}, T\right)$, $\beta(t) \geq 0 e$

$$
\phi(t) \leq \alpha(t)+\int_{t_{0}}^{t} \beta(s) \phi(s), \text { para } t_{0} \leq t \leq T
$$

então

$$
\phi(t) \leq \alpha(t)+\int_{t_{0}}^{t} \beta(s) \alpha(s) e^{\int_{s}^{t} \beta(u) d u} d s, \text { para } t_{0} \leq t \leq T .
$$

Teorema 1.1.6 (Desigualdade de Hölder) Sejam $f \in L^{p}(\Omega), g \in L^{q}(\Omega)$ com $1 \leq p, q \leq \infty e$ $\frac{1}{p}+\frac{1}{q}=1$. Então $f g \in L^{1}(\Omega)$

$$
\int_{\Omega}|f(x) g(x)| d x \leq\|f\|_{p}\|g\|_{q}
$$


Teorema 1.1.7 (Desigualdade de Hölder Generalizada) Sejam $1 \leq p_{1}, p_{2}, \cdots, p_{m} \leq \infty$, com $\frac{1}{p_{1}}+\frac{1}{p_{2}}+\cdots+\frac{1}{p_{m}}=1$. Se $f_{i} \in L^{p_{i}}(\Omega)$ para $i=1, \cdots$, m. Então

$$
\int_{\Omega}\left|f_{1} \cdots f_{m}\right| d s \leq \prod_{i=1}^{m}\left\|f_{i}\right\|_{p i}
$$

Teorema 1.1.8 (Desigualdade de Interpolação Para Normas em $L^{p}$ ) Suponha que $1 \leq s \leq r \leq$ $t \leq \infty e \frac{1}{r}=\frac{\theta}{s}+\frac{1-\theta}{t}$. Suponha também que $f \in L^{s}(\Omega) \cap L^{t}(\Omega)$. Então $\left.f \in L^{r}(\Omega)\right)$,e

$$
\|f\|_{r} \leq\|f\|_{s}^{\theta}\|f\|_{t}^{1-\theta}
$$

\subsection{Aspectos Básicos da Teoria de Semigrupos}

Nesta seção, recordaremos alguns resultados básicos sobre a teoria de $C_{0}$-semigrupos de operadores lineares. As demonstrações podem ser encontradas em Pazy [42] e mais condensadamente em Zheng [51]. Estes resultados serão utilizados no Capítulo 2.

\subsubsection{Semigrupos de operadores lineares de classe $C_{0}$}

Assumiremos que $(X,\|\|$.$) é um espaço de Banach.$

Definição 1.2.1 Uma família $\{S(t)\}_{t \geq 0}$, de operadores lineares limitados de X em X diz-se um semigrupo de operadores lineares limitados sobre X se:

(i) $S(0)=I$, (I é o operador identidade sobre $X)$

(ii) $S(t+s)=S(t) S(s)$ para todo $t, s \geq 0$. (a propriedade de semigrupo)

O operador linear definido por

$$
D(A)=\left\{x \in X: \lim _{t \rightarrow 0} \frac{S(t) x-x}{t} \text { existe }\right\}
$$

$\mathrm{e}$

$$
A x=\lim _{t \rightarrow 0} \frac{S(t) x-x}{t}=\left.\frac{d^{+} S(t) x}{d t}\right|_{t}=0 \text { para } x \in D(A),
$$


é chamado gerador infinitesimal do semigrupo $\{S(t)\}_{t \geq 0}, D(A)$ é chamado domínio de $A$.

Definição 1.2.2 Um semigrupo $\{S(t)\}_{t \geq 0}$ de operadores lineares limitados sobre $X$ é um semigrupo de operadores lineares limitados fortemente contínuo se

$$
\lim _{t \rightarrow 0} S(t) x=x \text { para todo } x \in X
$$

isto é,

$$
\lim _{t \rightarrow 0}\|S(t) x-x\|=0 \text { para cada } x \in X
$$

Um semigrupo de operadores lineares limitados fortemente contínuos sobre $X$ é chamado de semigrupos de classe $C_{0}$ ou simplesmente $C_{0}$-semigrupo.

Teorema 1.2.1 Seja $\{S(t)\}_{t \geq 0}$ um $C_{0}$ - semigrupo. Então existe uma constante $\omega \geq 0$ e $M \geq 1$ tal que

$$
\|S(t)\| \leq M e^{\omega t} \text { para } 0 \leq t<\infty .
$$

O proximo Teorema é um a caracterização para um $C_{0}$-semigrupo, que desempenha um papel crucial no estudo das equações diferenciais parciais (ver, por exemplo, [42]).

Teorema 1.2.2 Seja $\{S(t)\}_{t \geq 0}$ um $C_{0}$-semigrupo e seja A seu gerador infinitesimal. Então

(i) Para $x \in X, \lim _{h \rightarrow 0} \frac{1}{h} \int_{t}^{t+h} S(s) x d s=S(t) x$.

(ii) Para $x \in X, \int_{0}^{t} S(s) x d s \in D(A)$ e $A\left(\int_{0}^{t} S(s) x d s\right)=S(t) x-x$.

(iii) Para $x \in D(A), S(t) x \in D(A)$ e $\frac{d}{d t} S(t) x=A S(t) x=S(t) A x$.

(iv) Para $x \in D(A), S(t) x-S(s) x=\int_{S}^{t} S(\tau) A x d \tau=\int_{S}^{t} A S(\tau) x d \tau$.

Corolário 1.2.1 Se A é o gerador infinitesimal de um $C_{0}$-semigrupo $\{S(t)\}_{t \geq 0}$, então $D(A)$ é denso em X e A é um operador linear fechado.

Definição 1.2.3 Um semigrupo $\{S(t)\}_{t \geq 0}$ é chamado um semigrupo de contrações se existe uma constante $\alpha \in(0,1]$ tal que

$$
\|S(t) x-S(t) y\| \leq \alpha\|x-y\| \quad \text { para todo } \quad x, y \in X .
$$


Definição 1.2.4 Seja A um operador linear sobre $X$, não necessariamente limitado, o conjunto resolvente $\rho(A)$ de A é o conjunto de todos os números complexos $\lambda$ tal que $\lambda I-A$ é invertível e $(\lambda I-A)^{-1}$ é um operador linear limitado sobre X. A família $R(\lambda: A)=(\lambda I-A)^{-1}, \lambda \in \rho(A)$ de operadores lineares limitados é chamada resolvente de A. O conjunto $\sigma(A)=\mathbb{C} \backslash \rho(A)$ é chamado espectro de A.

\subsubsection{Teoremas de Hille-Yosida e de Lumer-Phillips}

O próximo teorema da uma condição necessária e suficiente para um operador densamente definido ser o gerador infinetesimal de um semigrupo linear de contrações em um espaço de Banach.

Teorema 1.2.3 (Hille-Yosida) Um operador (ilimitado) A é o gerador infinitesimal de um $C_{0^{-}}$ semigrupo de contrações $\{S(t)\}_{t \geq 0}$ se, e somente se,

(i) A é fechado e $\overline{D(A)}=X$.

(ii) O conjunto resolvente $\rho(A)$ de A contém $\mathbb{R}^{+}$e para todo $\lambda>0$

$$
\|R(\lambda: A)\| \leq \frac{1}{\lambda}
$$

A seguir, apresentamos outra caracterização dos geradores infinitesimais dos $C_{0}$-semigrupos de contrações, o teorema de Lumer-Phillips, que é muito útil na teoria dos semigrupos lineares. Para estabelecer tal teorema, recordemos o conceito de dissipatividade. Assumamos que $X^{*}$ é o espaço dual de $X$. Denotemos os valores de $x^{*} \in X^{*}$ em $x \in X$ por $\left\langle x^{*}, x\right\rangle$ ou $\left\langle x, x^{*}\right\rangle$.

Para cada $x \in X$, definimos a aplicação dualidade $J: X \rightarrow 2^{X^{*}}$,

$$
J(x)=\left\{x^{*} \in X^{*}: \operatorname{Re}\left\langle x, x^{*}\right\rangle=\|x\|_{X}^{2}=\left\|x^{*}\right\|_{X^{*}}^{2}\right\} .
$$

Do teorema de Hahn-Banach segue que $J(x) \neq \emptyset$ para todo $x \in X$.

Definição 1.2.5 Dizemos que o operador linear $A: D(A) \subset X \rightarrow X$ é dissipativo, quando

$$
\operatorname{Re}\left\langle A x, x^{*}\right\rangle \leq 0, \quad \text { para todo } \quad x \in D(A)
$$


Uma caracterização muito útil dos operadores dissipativos é dado pelo próximo resultado.

Teorema 1.2.4 Um operador linear $A: D(A) \subset X \rightarrow R(A) \subseteq X$ é dissipativo se, e somente se,

$$
\|(\lambda I-A) x\| \leq \lambda\|x\| \quad \text { para todo } \quad x \in D(A), \lambda>0 .
$$

Observação 1.2.1 A motivação para o uso da palavra "dissipativo"vem do caso onde X é um espaço de Hilbert. Então, para um operador linear A, a condição (1.2) é equivalente à condição

$$
R e(x, A x) \leq 0 \quad \text { para todo } \quad x \in D(A)
$$

onde $(\cdot, \cdot)$ denota o produto escalar interno em $X$.

Definição 1.2.6 Um operador linear $A: D(A) \subset X \rightarrow R(A) \subseteq X$ é m-dissipativo se A é dissipativo $e$

$$
R(\lambda I-A)=X \quad \text { para todo } \quad \lambda>0
$$

isto é, para qualquer $g \in X$, existe $f \in D(A)$ tal que $(\lambda I-A) f=g$.

Em virtude desta definição, podemos concluir

Corolário 1.2.2 Todo operador m-dissipativo é um operador dissipativo.

Agora podemos estabelecer o teorema de Lumer-Phillips.

Teorema 1.2.5 (Lumer-Phillips) Um operador linear A gera um $C_{0}$-semigrupo de contrações sobre $X$ se, e somente se,

(i) $D(A)$ é denso em $X$, $e$

(ii) A é m-dissipativo.

\subsubsection{Problema de valor inicial não homogêneo}

Consideremos agora o problema de valor inicial não homogêneo

$$
\left\{\begin{array}{l}
\frac{d u}{d t}-A u(t)=f(t, u(t)), t \geq 0 \\
u(0)=u_{0}
\end{array}\right.
$$


onde $A$ é gerador infinitesimal de um $C_{0}$-semigrupo $\{S(t)\}_{t \geq 0}$ sobre $X$ e $f:[0, T] \times X \rightarrow X$ é contínua em $t$ e satisfaz a condição de Lipschitz em $u$.

Definição 1.2.7 (Solução forte) Uma função u que é diferenciável sobre quase todo $[0, T]$ tal que $u^{\prime} \in L^{1}(0, T ; X)$ é chamada uma "solução forte" do problema de valor inicial (1.3) se u $(0)=$ $u_{0} e$

$$
u^{\prime}(t)=A U(t)+f(t, u(t)) \quad \text { quase sempre sobre } \quad[0, T]
$$

Definição 1.2.8 (Mild solution) Uma função $u \in C([0, T] ; X)$ dada por

$$
u(t)=S(t) x+\int_{0}^{t} S(t-s) f(s, u(s)) d s, 0 \leq t \leq T
$$

é chamada de "mild solution"do problema (1.3) sobre $[0, T]$.

O resultado clássico, a seguir, assegura a existência e unicidade de "mild solutions"de (1.3) para uma função Lipschitz contínua $f$.

Teorema 1.2.6 Seja $f:[0, \infty) \times X \rightarrow X$ contínua em t para $t \geq 0$ e localmente Lipschitz contínua em $u$, uniformemente em $t$ sobre intervalos limitados. Se A é o gerador infinitesimal de um $C_{0^{-}}$ semigrupo $\{S(t)\}_{t \geq 0}$ sobre $X$, então para todo $u_{0} \in X$ existe $t_{\max } \leq \infty$ tal que o problema de valor inicial

$$
\left\{\begin{array}{l}
u_{t}-A u(t)=f(t, u(t)), \quad t \geq 0, \\
u(0)=u_{0},
\end{array}\right.
$$

possui uma única mild solution u definida em $\left[0, t_{\max }\right)$. Além disso, se $t_{\max }<\infty$ então

$$
\lim _{t \rightarrow t_{\max }}\|u(t)\|_{X}=\infty
$$

Em geral se $f:[0, T] \times X \rightarrow X$ é apenas Lipschitz contínua em ambas as variáveis, a mild solution não é uma solução forte para o problema de valor inicial. Entretanto, se $X$ é reflexivo, a continuidade Lipschitz de $f$ assegura que a mild solution $u$ com dado inicial $u_{0} \in D(A)$ é uma solução forte. Este resultado é garantido pelo próximo teorema.

Teorema 1.2.7 Seja A o gerador infinitesimal de um $C_{0}$-semigrupo $\{S(t)\}$ sobre um espaço de Banach reflexivo $X$. Se $f:[0, T] \times X \rightarrow X$ é Lipschitz contínua em ambas as variáveis, $u_{0} \in D(A)$ 
e u é uma mild solution do problema de valor inicial (1.3), então u é uma solução forte deste problema de valor inicial.

Ball em [6] define solução fraca da equação (1.3) onde $A$ é um operador linear fechado sobre $X$ e $f \in L^{1}(0, T ; X)$ como segue:

Definição 1.2.9 (Solução fraca) Uma função $u \in C([0, T] ; X)$ é uma solução fraca de (1.3) sobre $[0, T]$ se para todo $v^{*} \in D\left(A^{*}\right)$ a função $\left\langle u(t), v^{*}\right\rangle$ é absolutamente contínua sobre $[0, T] e$

$$
\frac{d}{d t}\left\langle u(t), v^{*}\right\rangle=\left\langle u(t), A^{*} v^{*}\right\rangle+\left\langle f\left(t, u(t), v^{*}\right\rangle \quad \text { q.s. sobre } \quad[0, T]\right.
$$

Da definição acima, segue o seguinte resultado.

Teorema 1.2.8 Existe para cada $u_{0} \in X$ uma única solução fraca u de (1.3) sobre $[0, T]$ satisfazendo $u(0)=u_{0}$ se, e somente se, A é o gerador infinitesimal de um $C_{0}$-semigrupo de operadores lineares limitados $\{S(t)\}$ sobre $X$, e neste caso u é dada por

$$
u(t)=S(t) u_{0}+\int_{0}^{t} S(t-s) f(s, u(s)) d s, \quad 0 \leq t \leq T
$$

Estes resultados podem ser encontrados em Pazy [42] e Ball [6].

\subsection{Atrator Global e Atrator Exponencial}

Nesta seção definimos atrator global e apresentamos seus resultados básicos. As demonstrações podem ser encontradas em [16], [48] e [51]. Terminamos esta seção definindo conjunto inércia e suas principais propriedades. As demonstrações dos resultados sobre conjunto inércia podem ser encontradas em [22].

\subsubsection{Definição de atrator global}

Definição 1.3.1 Suponha que E é um espaço métrico completo, e $S(t)$ é um $C_{0}$-semigrupo de operadores não lineares definidos sobre E. Um conjunto $\mathscr{A} \subset E$ diz-se um atrator global se as seguintes propriedades são satisfeitas: 
(i) Á é um conjunto positivamente invariante, isto é,

$$
S(t) \mathscr{A}=\mathscr{A}, \forall t \geq 0
$$

(ii) $\mathscr{A}$ é compacto em $E$.

(iii) $\mathscr{A}$ atrai uniformemente todo limitado de $E$, isto é, para qualquer limitado $B \subset E$

$$
\operatorname{dist}(S(t) B, \mathscr{A})=\sup _{x \in S(t) B} \inf _{y \in \mathscr{A}} d(x, y) \rightarrow 0 \text { com } t \rightarrow \infty .
$$

Para estabelecer a existência de um atrator global, um passo crucial é mostrar a existência de um conjunto absorvente que definimos a seguir.

Definição 1.3.2 Um semigrupo $\{S(t)\}_{t \geq 0}$ possui um conjunto absorvente $B_{0}$ em um espaço métrico $E$, se para qualquer conjunto limitado $B \subset E$, existe $t_{0}=t_{0}(B) \geq 0$ tal que

$$
S(t) B \subset B_{0} \forall t \geq t_{0}
$$

Definição 1.3.3 Seja $A \subset E$. Definimos o conjunto $\omega$-limite $\omega(A)$ por

$$
\omega(A)=\cap_{s \geq 0} \overline{\cup_{t \geq s} S(t) A}
$$

onde o fecho tomado em E. Equivalentemente, $\omega(A)$ pode ser definido como

$$
\omega(A)=\left\{\phi: \exists t_{n} \rightarrow \infty \text { e uma sequência } \phi_{n} \in A \text { tal que } S\left(t_{n}\right) \phi_{n} \rightarrow \phi \text { com } n \rightarrow \infty\right\}
$$

Podemos ver das definições de atrator global e conjunto absorvente para um $C_{0}$-semigrupo não linear $S(t)$, que a existência de um atrator global implica a existência de um conjunto absorvente. A reciproca nem sempre é válida, pois um conjunto absorvente não necessáriamente é um conjunto invariante. Entretanto, temos o seguinte resultado que será fortemente usado nos próximos capítulos para provar a existência de atrator global.

Teorema 1.3.1 ([3], [48]) Suponha que $\{S(t)\}_{t \geq 0}$ é um não linear $C_{0}$-semigrupo definido sobre um espaço de Banach E satisfazendo as seguintes condições: 
(i) Conjunto Absorvente: Existe um conjunto absorvente limitado $B_{0}$.

(ii) Compacidade Assintótica: Para qualquer sequência limitada $\left\{\left(y_{n}\right)\right\} \subset E$ e uma sequência numérica $t_{n} \rightarrow \infty$, existe uma subsequência $\left\{S\left(t_{n_{k}}\right)\left(y_{n_{k}}\right)\right\}$ de $\left\{S\left(t_{n}\right)\left(y_{n}\right)\right\}$ que é convergente em $E$.

Então $S(t)$ Possui um atrator global $\mathscr{A} \subset E$, que é precisamente o conjunto $\omega$-limite de $B_{0}$.

Nos modelos que vamos estudar, para provarmos a propriedade $(i)$ do Teorema 1.3.1, isto é, a existência de um conjunto absorvente, utilizaremos o lema de Nakao ([37], Lema 2.1).

Lema 1.3.1 (Nakao) Seja $\phi$ uma função contínua não negativa sobre $[0, T), T>1$, possivelmente $T=\infty$, satisfazendo

$$
\sup _{t \leq s \leq t+1} \phi(s)^{1+\gamma} \leq k_{0}(\phi(t)-\phi(t+1))+k_{1}, \quad 0 \leq t \leq T-1
$$

para algum $k_{0}>0, k_{1}>0$ e $\gamma>0$. Então temos

$$
\phi(t) \leq\left(k_{0}^{-1} \gamma(t-1)^{+}+\left(\sup _{0 \leq s \leq 1} \phi(s)\right)^{-\gamma}\right)^{\frac{-1}{\gamma}}+k_{1}^{\frac{1}{\gamma+1}}, \quad 0 \leq t<T
$$

Se (1.4) vale com $\gamma=0$ temos, em vez de (1.5),

$$
\phi(t) \leq \sup _{0 \leq s \leq 1} \phi(s)\left(\frac{1+k_{0}}{k_{0}}\right) e^{-\theta t}+k_{1}, \quad 0 \leq t \leq T,
$$

onde $\theta=\ln \left(\frac{1+k_{0}}{k_{0}}\right)$.

Por outro lado, para verificarmos a propriedade (ii) de Teorema 1.3.1, também conhecida como compacidade assintótica, utilizaremos uma abordagem mais recente, que pode ser encontradas em Khanmamedov [25] ou Lasiecka-Chueshov ([16], Capítulo 2, Proposição 2.10).

Lema 1.3.2 Seja $\{S(t)\}_{t \geq 0}$ um semigrupo sobre um espaço métrico $(E, d)$ que possui um conjunto absorvente $B_{0}$. Além disso, assuma que para qualquer $\varepsilon \geq 0$ existe $T=T\left(B_{0}, \varepsilon\right)$ tal que

$$
d\left(S(T) y_{1}, S(T) y_{2}\right) \leq \varepsilon+\phi_{T}\left(y_{1}, y_{2}\right), \quad \text { para todo } \quad y_{1}, y_{2} \in B_{0}
$$


onde $\phi_{T}\left(y_{1}, y_{2}\right)$ é uma função contrativa sobre $B_{0} \times B_{0}$, no seguinte sentido, para qualquer sequência $\left\{y_{n}\right\} \subset B_{0}$,

$$
\liminf _{n \rightarrow \infty} \limsup _{m \rightarrow \infty} \phi_{T}\left(y_{n}, y_{m}\right)=0 .
$$

Então, $\{S(t)\}_{t \geq 0}$ é compactamente assintótico em E, isto é,para qualquer sequência limitada $\left(y_{n}\right) \subset E$ e $t_{n} \rightarrow \infty, S\left(t_{n}\right) y_{n}$ é precompacto em $E$.

\subsubsection{Dimensão fractal e dimensão de Hausdorff}

Seja $Y$ um subconjunto de um espaço métrico $E$. Dado $d \in \mathbb{R}_{+}$e $\varepsilon>0$, definimos

$$
\mu_{H}(Y, d, \varepsilon)=\inf \sum_{i \in I} r_{i}^{d}
$$

o ínfimo de todas as coberturas de $Y$ por uma família $\left(B_{i}\right)_{i \in I}$ de bolas de $E$ de raio $r_{i} \leq \varepsilon$.

Vê-se claramente que $\mu_{H}(Y, d, \varepsilon)$ é uma função não crescente de $\varepsilon$ e o número $\mu_{H}(Y, d) \in$ $[0, \infty]$ definido por

$$
\mu_{H}(Y, d)=\lim _{\varepsilon \rightarrow 0} \mu_{H}(Y, d, \varepsilon)=\sup _{\varepsilon>0} \mu_{H}(Y, d, \varepsilon)
$$

é a medida d-dimensional de Hausdorff de $Y$. Claramente vê-se que, se $\mu_{H}\left(Y, d^{\prime}\right)<\infty$ para algum $d^{\prime}$, então $\mu_{H}(Y, d)=0$ para todo $d>d^{\prime}$. Assim podemos afirmar que existe $d_{0} \in[0, \infty]$ tal que $\mu_{H}(Y, d)=0$ para $d>d_{0}$ e $\mu_{H}(Y, d)=\infty$ para $d<d_{0}$, enquanto $\mu_{H}\left(Y, d_{0}\right)$ pode ser qualquer número em $[0, \infty]$. Este número $d_{0}$ é chamado a dimensão de Hausdorff de $Y$ e é denotado por $d_{H}(Y)$.

Seja $\eta_{\gamma}(\varepsilon)$ o número mínimo de bolas de $H$ de raio $\varepsilon$ que é necessário para cobrir $Y$. Chamamos de dimensão fractal de $Y$ ou compacidade de $Y$ o número

$$
d_{F}(Y)=\lim _{\varepsilon \rightarrow 0} \sup \frac{\log \eta_{\gamma}(\varepsilon)}{\log 1 / \varepsilon}
$$

Uma expressão alternativa para $d_{F}(Y)$ é dada por

$$
d_{F}(Y)=\inf \left\{d>0, \mu_{F}(Y, d)=0\right\},
$$

onde $\mu_{F}(Y, d)=\lim _{\varepsilon \rightarrow 0} \sup \varepsilon^{d} \eta_{\gamma}(\varepsilon)$.

A diferença entre a dimensão de Hausdorff e a dimensão fractal está no fato de que consid- 
eramos, em um caso, as coberturas de $Y$ por bolas de raio $\leq \varepsilon$, e no outro caso, a cobertura de $Y$ é dada por bolas de raio $\varepsilon$.

Para mais resultados a respeito de dimensão de Hausdorff e fractal ver Chueshov e Lasiecka [16] e Temam [48].

\subsubsection{Atrator exponencial (conjunto inércia)}

Para sistemas de equações diferenciais cujas soluções existem para todo $t \geq 0$, Eden et al. [22] introduziu a noção de atrator exponencial ou conjunto inércia, que é um conjunto de dimensão finita que contém o atrator global e, ao mesmo tempo, atrai exponencialmente todas as soluções do sistema.

Seja $E$ um espaço de Hilbert separável e $\mathscr{X}$ um subconjunto compacto de $E$. Seja $\{S(t)\}_{t \geq 0}$ um $C_{0}$ - semigrupo não linear que deixa $\mathscr{X}$ invariante e seja $\mathscr{A}=\cap\{S(t) \mathscr{X}\}_{t \geq 0}$, isto é, $\mathscr{A}$ é o atrator global para $\{S(t)\}_{t \geq 0}$ sobre $\mathscr{X}$.

Definição 1.3.4 Um conjunto compacto $\mathscr{M}$ é chamado de atrator exponencial ou conjunto inércia para o $C_{0}$-semigrupo $\{S(t)\}_{t \geq 0}$ se satisfaz as seguintes propriedades:

(i) $\mathscr{A} \subset \mathscr{M} \subset \mathscr{X}$, onde $\mathscr{A}$ é o atrator global associado a $\{S(t)\}_{t \geq 0}$,

(ii) $S(t) \mathscr{M} \subset \mathscr{M}$, para todo $t \geq 0$,

(iii) para $u_{0}$ em $\mathscr{X}$, dist $\left(S(t) u_{0}, \mathscr{M}\right) \leq c_{1} e^{-c_{2} t}$ para todo $t \geq 0$, onde $c_{1}$ e $c_{2}$ são independentes de $u_{0}$,

(iv) $\mathscr{M}$ possui dimensão fractal finita, $d \mathscr{M}$.

Uma condição suficiente para a existência de um atrator exponencial é baseada num princípio de dicotomia chamado de propriedade de compressão (squeezing property) que é definido como segue.

Definição 1.3.5 Um semigrupo contínuo $\{S(t)\}_{t \geq 0}$ satisfaz a propriedade de compressão (squeezing property) sobre $\mathscr{X}$ se existe $t_{*}>0$ tal que $S_{*}=S\left(t_{*}\right)$ satisfaz: existe uma projeção ortogonal $P$ de posto $N_{0}$ tal que para todo u e v em $\mathscr{X}$, ou

$$
\left\|(I-P)\left(S_{*} u-S_{*} v\right)\right\|_{E} \leq\left\|P\left(S_{*} u-S_{*} v\right)\right\|_{E}
$$


ou então

$$
\left\|S_{*} u-S_{*} v\right\|_{E} \leq \frac{1}{8}\|u-v\|_{E} .
$$

A existência de um atrator exponencial segue então do próximo teorema. (cf. Eden et al [22], Teorema 3.1).

Teorema 1.3.2 (Existência de Atrator Exponencial) Se $\{S(t)\}_{t \geq 0}$ satisfaz a propriedade de compressão sobre $\mathscr{X}$ e $S_{*}=S\left(t_{*}\right)$ é Lipschitz sobre $\mathscr{X}$ com constante de Lipschitz L, então existe um atrator exponencial $\mathscr{M}$ para $\{S(t)\}_{t \geq 0}$ tal que

$$
d_{\mathscr{M}} \leq N_{0} \max \{1, \ln (16 L+1) / \ln 2\}
$$

$e$

$$
\operatorname{dist}_{E}\left(S(t) u_{0}, \mathscr{M}\right) \leq c_{1} e^{\left(-c_{2} / t_{*}\right) t}
$$

Note que, precisamos apenas encontrar o tempo $t_{*}$ e a projeção $P$ de posto $n_{0}$ a fim de avaliar o lado direito das desigualdades do teorema. 


\section{Solução Global para o Problema com}

\section{Dissipação Linear}

Seja $\Omega \subset \mathbb{R}^{N}, N \geq 1$, um aberto e limitado com fronteira suave $\Gamma=\partial \Omega$. Consideremos em $\Omega \times \mathbb{R}^{+}$a equação da viga não linear do tipo Kirchhoff dada pela seguinte equação

$$
\left\{\begin{array}{l}
u_{t t}+\Delta^{2} u-M\left(\|\nabla u(t)\|_{2}^{2}\right) \Delta u+\beta u_{t}+|u|^{\rho} u=h(x, t) \\
u=\frac{\partial u}{\partial v}=0 \quad \text { sobre } \quad \Gamma \\
u(x, 0)=u_{0}(x), u_{t}(x, 0)=u_{1}(x) \quad \text { sobre } \Omega
\end{array}\right.
$$

onde $M$ é uma função real não negativa de classe $C^{1}, \beta>0$,

$$
0<\rho \leq \frac{2}{N-2} \text { se } N \geq 3 \text { e } \rho>0 \text { se } N=1,2
$$

e $h$ é uma força externa.

Para obtermos os resultados de existência de solução global assumiremos que a função $M$ satisfaz as seguintes hipóteses:

(i) $M$ é uma função de classe $C^{1}([0,+\infty))$ tal que

$$
M(s) \geq 0 \text { para todo } s \in \mathbb{R}^{+},
$$


(ii) $M$ é monótona crescente, o que implica que

$$
M(s) s \geq \widehat{M}(s), \text { onde } \widehat{M}(\lambda)=\int_{0}^{\lambda} M(s) d s
$$

\subsection{Existência de Solução Global}

Nesta seção investigaremos a existência e unicidade de soluções para o problema (2.1). A existência de solução forte será analisada no espaço de fase $E_{1}=\left(H^{4}(\Omega) \cap H_{0}^{2}(\Omega)\right) \times H_{0}^{2}(\Omega)$ e a existência de solução fraca no espaço de fase $E_{0}=H_{0}^{2}(\Omega) \times L^{2}(\Omega)$.

\subsubsection{Existência de solução forte}

Consideremos o espaço de Hilbert

$$
E_{1}=\left(H^{4}(\Omega) \cap H_{0}^{2}(\Omega)\right) \times H_{0}^{2}(\Omega)
$$

munido da norma

$$
\|(u, v)\|_{E_{1}}^{2}=\left\|\Delta^{2} u\right\|_{2}^{2}+\|\Delta u\|_{2}^{2}+\|\Delta v\|_{2}^{2}
$$

O resultado que garante a existência de solução forte é dado pelo seguinte teorema.

Teorema 2.1.1 Suponha que a função M satisfaz as condições (2.3) e (2.4). Então, se

$$
h \in C^{1}\left([0, \infty), L^{2}(\Omega)\right) \quad e \quad\left(u_{0}, u_{1}\right) \in E_{1},
$$

o problema (2.1) admite uma única solução global u nas seguintes classes

$$
\begin{array}{r}
u \in L^{\infty}\left(0, \infty ; H^{4}(\Omega) \cap H_{0}^{2}(\Omega)\right), \\
u_{t} \in L^{\infty}\left(0, \infty ; H_{0}^{2}(\Omega)\right), \\
u_{t t} \in L^{\infty}\left(0, \infty ; L^{2}(\Omega)\right) .
\end{array}
$$

\section{Demonstração:}

Primeiramente, mostraremos a existência e unicidade de solução local para o problema (2.1) usando resultados da teoria de semigrupos que pode ser encontrada em [42]. Em seguida, obte- 
mos solução global através de estimativas uniformes sobre a solução do problema no espaço energia $E_{1}=H^{4}(\Omega) \cap H_{0}^{2}(\Omega) \times H_{0}^{2}(\Omega)$.

Com efeito, fazendo $v=u_{t}$ a equação de (2.1) pode ser transformada no seguinte sistema de primeira ordem:

$$
\left\{\begin{array}{l}
u_{t}-v=0 \\
v_{t}+\Delta^{2} u-M\left(\|\nabla u(t)\|_{2}^{2}\right) \Delta u+\beta v+|u|^{\rho} u=h
\end{array}\right.
$$

Introduzindo o vetor $U=(u, v)$, o sistema (2.6) pode ainda ser reescrito da seguinte forma

$$
U_{t}=A U+F(U), \text { onde } A U=\left(\begin{array}{rc}
0 & I \\
-\Delta^{2} & -\beta
\end{array}\right)\left(\begin{array}{l}
u \\
v
\end{array}\right)
$$

$\mathrm{e}$

$$
F(U)=(0, \psi(u)), \quad \operatorname{com} \psi(u)=M\left(\|\nabla u\|_{2}^{2}\right) \Delta u-|u|^{\rho} u+h
$$

Assim, a equação (2.1) fica expressa pelo sistema de primeira ordem dado por

$$
U_{t}-A U=F(U)
$$

com condições de fronteira

$$
\left.U\right|_{\Gamma}=\left.(u, v)\right|_{\Gamma}=0
$$

e condições iniciais

$$
U(0)=U_{0}=\left(u_{0}, u_{1}\right)
$$

Prosseguindo, definamos o espaço de Hilbert

$$
E_{0}=H_{0}^{2}(\Omega) \times L^{2}(\Omega)
$$


equipado com o seguinte produto interno

$$
(U, V)_{E_{0}}=\left(\left(\begin{array}{c}
u \\
v
\end{array}\right),\left(\begin{array}{c}
w \\
z
\end{array}\right)\right)_{E_{0}}=\int_{\Omega} \Delta u \Delta w d x+\int_{\Omega} v z d x
$$

e norma

$$
\|(u, v)\|_{E_{0}}^{2}=\|\Delta u\|_{2}^{2}+\|v\|_{2}^{2}
$$

Para o operador matriz $A: D(A) \subset E_{0} \rightarrow E_{0}$, temos

$$
D(A)=E_{1}=H^{4}(\Omega) \cap H_{0}^{2}(\Omega) \times H_{0}^{2}(\Omega) .
$$

Observe que encontrar uma solução de (2.1) é equivalente a encontrar uma solução para (2.7)-(2.9).

Lema 2.1.1 A parte linear de (2.7) gera um $C_{0}$-semigrupo de operadores $\{S(t)\}_{t \geq 0}$.

\section{Demonstração:}

Para mostrar que a parte linear do sistema (2.7) gera um $C_{0}$-semigrupo devemos mostrar que o operador $A: D(A) \subset E_{0} \longrightarrow E_{0}$ satisfaz as seguintes propriedades:

(i) $D(A)$ é denso em $E_{0}$.

(ii) $A$ é $m$-dissipativo ( $A$ dissipativo e $\left.R(I-A)=E_{0}\right)$.

Claramente, $D(A)$ é denso em $E_{0}$. Resta provar que $A$ é $m$-dissipativo.

A propriedade de dissipatividade pode ser vista pela seguinte desigualdade,

$$
(A U, U)_{E_{0}}=\int_{\Omega} \Delta u \Delta v d x+\int_{\Omega}\left(-\Delta^{2} u-\beta v\right) v d x=-\beta\|v\|_{2}^{2} \leq 0 .
$$

Agora, para mostrar que $R(I-A)=E_{0}$, considere a equação

$$
(I-A)\left(\begin{array}{c}
u \\
v
\end{array}\right)=\left(\begin{array}{c}
\mathscr{F} \\
\mathscr{G}
\end{array}\right)
$$


onde $\mathscr{F} \in H_{0}^{2}(\Omega)$ e $\mathscr{G} \in L^{2}(\Omega)$. Podemos reescrever (2.11) como

$$
\left\{\begin{array}{l}
u-v=\mathscr{F} \\
\Delta^{2} u+(\beta+1) v=\mathscr{G} .
\end{array}\right.
$$

Substituindo $v$ obtido pela primeira equação na segunda equação encontramos

$$
\Delta^{2} u+(\beta+1) u=(\beta+1) \mathscr{F}+\mathscr{G} \in L^{2}(\Omega)
$$

Multiplicando (2.13) por $w \in H_{0}^{2}(\Omega)$ e integrando sobre $\Omega$, obtemos

$$
\int_{\Omega}(\Delta u \Delta w+(\beta+1) u w) d x=\int_{\Omega}((\beta+1) \mathscr{F}+\mathscr{G}) w d x
$$

Agora, definindo $a: H_{0}^{2}(\Omega) \times H_{0}^{2}(\Omega) \rightarrow \mathbb{R}$ por

$$
a(u, w)=\int_{\Omega}(\Delta u \Delta w+(\beta+1) u w) d x
$$

temos que $a(u, w)$ é uma forma bilinear, contínua e coerciva. Logo, pelo teorema de LaxMilgram [12], para $\varphi=((\beta+1) \mathscr{F}+\mathscr{G}) \in L^{2}(\Omega) \hookrightarrow\left(H_{0}^{2}(\Omega)\right)^{\prime}$ existe uma única $u \in H_{0}^{2}(\Omega)$ tal que

$$
a(u, w)=\int_{\Omega}((\beta+1) \mathscr{F}+\mathscr{G}) w d x, \quad \forall w \in H_{0}^{2}(\Omega) .
$$

Pela regularidade de problemas elípticos podemos concluir que o problema (2.13) admite uma única solução $u$ tal que

$$
u \in H^{4}(\Omega) \cap H_{0}^{2}(\Omega) .
$$

Então deduzimos da primeira equação de (2.12) que $v-u=\mathscr{F} \in H_{0}^{2}(\Omega)$. Assim

$$
R(I-A)=H_{0}^{2}(\Omega) \times L^{2}(\Omega)
$$

e portanto, $A$ é $m$-dissipativo.

Assim, pelo teorema de Lumer-Phillips, a parte linear do sistema (2.7) gera um $C_{0}$-semigrupo de contrações $\{S(t)\}_{t \geq 0}$ e isto conclue a prova do Lema 2.1.1. 
Agora, para mostrar existência local de solução para (2.7)-(2.8) é suficiente mostrar que o lado direito de (2.7) é uma função localmente Lipschitz. Isto é garantido pelo seguinte lema.

Lema 2.1.2 Suponha que $M$ satisfaz as condições (2.3) e (2.4), com $0 \leq t \leq T_{0}$. Então o operador $F(U)$ é localmente Lipschitz em $E_{0}$.

\section{Demonstração:}

Sejam $u, \widetilde{u} \in H_{0}^{2}(\Omega), w \in L^{2}(\Omega)$ e $R=R\left(T_{0}\right)>0$ com $\|u\|_{H_{0}^{2}} \leq R$ e $\|\widetilde{u}\|_{H_{0}^{2}} \leq R$. Note que

$$
\begin{aligned}
|(\psi(u)-\psi(\widetilde{u}), w)| \leq & \left|M\left(\|\nabla u\|^{2}\right)(\Delta u, w)-M\left(\|\nabla \widetilde{u}\|^{2}\right)(\Delta \widetilde{u}, w)\right| \\
& +\left|\int_{\Omega}\left(|u|^{\rho} u-|\widetilde{u}|^{\rho} \widetilde{u}\right) w d x\right| .
\end{aligned}
$$

Como a função $g(s)=|s|^{\rho} s$ é de classe $C^{1}$ e $g^{\prime}(s)=(\rho+1)|s|^{\rho}$, dados $\xi_{1}, \xi_{2} \in \mathbb{R}$, pelo Teorema do Valor Médio, existe $\left.\xi=\xi_{1}+\theta\left(\xi_{2}-\xi_{1}\right), \theta \in\right] 0,1[$ tal que

$$
\begin{gathered}
\left|g\left(\xi_{2}\right)-g\left(\xi_{1}\right)\right| \leq\left|g^{\prime}(\xi)\right|\left|\xi_{2}-\xi_{1}\right| \\
\left.\Rightarrow|| \xi_{2}\right|^{\rho} \xi_{2}-\left|\xi_{1}\right|^{\rho} \xi_{1}|\leq(\rho+1)| \xi^{\rho}\left|\xi_{2}-\xi_{1}\right| \\
\left.\Rightarrow|| \xi_{2}\right|^{\rho} \xi_{2}-\left|\xi_{1}\right|^{\rho} \xi_{1}|\leq(\rho+1)| \xi_{1}+\left.\left(\xi_{2}-\xi_{1}\right) \theta\right|^{\rho}\left|\xi_{2}-\xi_{1}\right| .
\end{gathered}
$$

Em particular, se $\xi_{1}=u$ e $\xi_{2}=\widetilde{u}$, temos

$$
\begin{aligned}
\left.|| u\right|^{\rho} u-|\widetilde{u}|^{\rho} \widetilde{u} \mid & \leq(\rho+1)|u+(\widetilde{u}-u) \theta|^{\rho}|\widetilde{u}-u| \\
& \leq(\rho+1)\{|u|+|\widetilde{u}|+|u|\}^{\rho}|u-\widetilde{u}| \\
& \leq(\rho+1)\{2|u|+2 \mid \widetilde{u}\}^{\rho}|u-\widetilde{u}| \\
& \leq(\rho+1) 2^{\rho}\{|u|+\mid \widetilde{u}\}^{\rho}|u-\widetilde{u}| \\
& \leq(\rho+1) 2^{\rho} 2^{\rho}\left\{|u|^{\rho}+|\widetilde{u}|^{\rho}\right\}|u-\widetilde{u}| .
\end{aligned}
$$

Logo

$$
|g(u)-g(\widetilde{u})| \leq 2^{2 \rho}(\rho+1)\left\{|u|^{\rho}+|\widetilde{u}|^{\rho}\right\}|u-\widetilde{u}| .
$$


Assim, usando a desigualdade de Holder $\frac{1}{2(\rho+1)}+\frac{\rho}{2(\rho+1)}+\frac{\rho}{2(\rho+1)}=1$ e observando que $H_{0}^{2}(\Omega) \hookrightarrow H_{0}^{1}(\Omega) \hookrightarrow L^{2(\rho+1)}(\Omega)$, deduzimos que

$$
\begin{aligned}
& \left|\int_{\Omega}\left(|u|^{\rho} u-|\widetilde{u}|^{\rho} \widetilde{u}\right) w d x\right| \\
& \quad \leq 2^{2 \rho}(\rho+1)\left\{\|u\|_{2(\rho+1)}^{\rho}+\|\widetilde{u}\|_{2(\rho+1)}^{\rho}\right\}\|u-\widetilde{u}\|_{2(\rho+1)}\|w\|_{2} \\
& \quad \leq 2^{2 \rho}(\rho+1) C_{1}\left\{\|u\|_{H_{0}^{2}}^{\rho}+\|\widetilde{u}\|_{H_{0}^{2}}^{\rho}\right\}\|u-\widetilde{u}\|_{H_{0}^{2}(\Omega)}\|w\|_{2} .
\end{aligned}
$$

Por outro lado, como $M$ satisfaz (2.3) e (2.4) então, a desigualdade triangular e o Teorema do Valor Médio implica

$$
\begin{aligned}
\mid M & \left(\|\nabla u\|_{2}^{2}\right)(\Delta u, w)-M\left(\|\nabla \widetilde{u}\|_{2}^{2}\right)(\Delta \widetilde{u}, w) \mid \\
\leq & \left|M\left(\|\nabla u\|_{2}^{2}\right)(\Delta(u-\widetilde{u}), w)\right|+\left|\left[M\left(\|\nabla u\|_{2}^{2}\right)-M\left(\|\nabla \widetilde{u}\|_{2}^{2}\right)\right](\Delta \widetilde{u}, w)\right| \\
\leq & \max _{0 \leq s \leq R} M(s)\|u-\widetilde{u}\|_{H_{0}^{2}}\|w\|_{2} \\
& +\max _{0 \leq s \leq R}\left|M^{\prime}(s)\right|\left|\|\nabla u\|_{2}^{2}-\|\nabla \widetilde{u}\|_{2}^{2}\right|\|\widetilde{u}\|_{H_{0}^{2}}\|w\|_{2} \\
\leq & \max _{0 \leq s \leq R} M(s)\|u-\widetilde{u}\|_{H_{0}^{2}}\|w\|_{2} \\
& +\max _{0 \leq s \leq R}\left|M^{\prime}(s)\right|\left|\|\nabla u\|_{2}+\|\nabla \widetilde{u}\|_{2}\right|\left|\|\nabla u\|_{2}-\|\nabla \widetilde{u}\|_{2}\right|\|\widetilde{u}\|_{H_{0}^{2}}\|w\|_{2} \\
\leq & \max _{0 \leq s \leq R} M(s)\|u-\widetilde{u}\|_{H_{0}^{2}}\|w\|_{2} \\
& +\max _{0 \leq s \leq R}\left|M^{\prime}(s)\right| C_{2}\left[\left(\|u\|_{H_{0}^{2}}+\|\widetilde{u}\|_{H_{0}^{2}}\right)\|\widetilde{u}\|_{H_{0}^{2}}\right]\|u-\widetilde{u}\|_{H_{0}^{2}}\|w\|_{2} .
\end{aligned}
$$

Assim, substituindo (2.16) e (2.17) em (2.15), temos

$$
\frac{|(\psi(u)-\psi(\widetilde{u}), w)|}{\|w\|_{2}} \leq C\left(T_{0},\|u\|_{H_{0}^{2}},\|\widetilde{u}\|_{H_{0}^{2}}\right)\|u-\widetilde{u}\|_{H_{0}^{2}}
$$

$\mathrm{ou}$

$$
\|\psi(u)-\psi(\widetilde{u})\|_{\left(L^{2}(\Omega)\right)^{\prime}} \leq C\left(T_{0},\|u\|_{H_{0}^{2}},\|\widetilde{u}\|_{H_{0}^{2}}\right)\|u-\widetilde{u}\|_{H_{0}^{2}}
$$

$\operatorname{com} C\left(T_{0},\|u\|_{H_{0}^{2}},\|\widetilde{u}\|_{H_{0}^{2}}\right)>0$. 
Portanto,

$$
\begin{aligned}
\|F(U)-F(\widetilde{U})\|_{E_{0}} & =\|\psi(u)-\psi(\widetilde{u})\|_{2} \\
& =\|\psi(u)-\psi(\widetilde{u})\|_{2^{\prime}} \\
& \leq C\left(T_{0},\|u\|_{H_{0}^{2}},\|\widetilde{u}\|_{H_{0}^{2}}\right)\|u-\widetilde{u}\|_{H_{0}^{2}} \\
& \leq C\left(T_{0},\|U\|_{E_{0}},\|\widetilde{U}\|_{E_{0}}\right)\|U-\widetilde{U}\|_{E_{0}} .
\end{aligned}
$$

que prova que $F$ é localmente Lipschitz em $E_{0}$. E portanto concluímos a prova do Lema 2.1.2

Do Lema 2.1.1 e Lema 2.1.2 segue que o problema (2.7) e consequentemente (2.1) possui uma solução local $U=\left(u, u_{t}\right)$ nas seguintes classes

$$
\left(u, u_{t}\right) \in L^{\infty}\left(0, T_{\max } ; H^{4}(\Omega) \cap H_{0}^{2}(\Omega) \times H_{0}^{2}(\Omega)\right) .
$$

Resta mostrar a existência global. Para isto é suficiente provar que a $U=\left(u, u_{t}\right)$ é uniformemente limitada.

Lema 2.1.3 Seja $U=(u, v)$ uma solução de (2.7) em seu intervalo maximal $\left[0, T_{\text {max }}\right)$. Então

$$
\lim _{t \rightarrow T_{\max }}\left\{\left\|\Delta^{2} u(t)\right\|_{2}^{2}+\left\|\Delta u_{t}(t)\right\|_{2}^{2}\right\}<\infty
$$

\section{Demonstração:}

Multiplicando a equação em (2.1) por $u_{t}$, então integrando em $\Omega$ encontramos

$$
\begin{aligned}
\frac{1}{2} & \frac{d}{d t}\left\{\left\|u_{t}(t)\right\|_{2}^{2}+\|\Delta u(t)\|_{2}^{2}+\widehat{M}\left(\|\nabla u(t)\|_{2}^{2}\right)+\frac{2}{\rho+2}\|u(t)\|_{\rho+2}^{\rho+2}\right\}+\beta\left\|u_{t}(t)\right\|_{2}^{2} \\
& =\int_{\Omega} h u_{t} d x
\end{aligned}
$$

Integrando de 0 até $t \leq T_{\max }$ obtemos

$$
\begin{gathered}
\left\|u_{t}(t)\right\|_{2}^{2}+\|\Delta u(t)\|_{2}^{2}+\widehat{M}\left(\|\nabla u(t)\|_{2}^{2}\right)+\frac{2}{\rho+2}\|u(t)\|_{\rho+2}^{\rho+2}+\beta \int_{0}^{t}\left\|u_{t}(s)\right\|_{2}^{2} d s \\
\leq \frac{1}{\beta} \int_{0}^{t}\|h(s)\|_{2}^{2} d s+\left\|u_{1}\right\|_{2}^{2}+\left\|\Delta u_{0}\right\|_{2}^{2}+\widehat{M}\left(\left\|\nabla u_{0}\right\|_{2}^{2}\right)+\frac{2}{\rho+2}\left\|u_{0}\right\|_{\rho+2}^{\rho+2} .
\end{gathered}
$$


Usando que $\left(u_{0}, u_{1}\right) \in D(A) \subset E_{0}$ e $h \in C^{1}\left([0, \infty), L^{2}(\Omega)\right)$, existe uma constante $C_{3}>0$ tal que

$$
\frac{1}{\beta} \int_{0}^{t}\|h(s)\|_{2}^{2} d s+\left\|u_{1}\right\|_{2}^{2}+\left\|\Delta u_{0}\right\|_{2}^{2}+\widehat{M}\left(\left\|\nabla u_{0}\right\|_{2}^{2}\right)+\frac{2}{\rho+2}\left\|u_{0}\right\|_{\rho+2}^{\rho+2} \leq C_{3}
$$

Como $\widehat{M}\left(\|\nabla u(t)\|_{2}^{2}\right) \geq 0$, segue que

$$
\|U(t)\|_{E_{0}}^{2}=\left\|\left(u(t), u_{t}(t)\right)\right\|_{E_{0}}^{2} \leq C_{3}, \forall t \in\left[0, T_{\max }\right)
$$

No que segue, para obter estimativas de ordens maiores diferenciamos a equação (2.1) com respeito a $t$ e multiplicando o resultado por $u_{t t}$, e integrando sobre $\Omega$, vemos

$$
\begin{aligned}
\frac{1}{2} \frac{d}{d t}\left\{\left\|u_{t t}(t)\right\|_{2}^{2}+\left\|\Delta u_{t}(t)\right\|_{2}^{2}\right\} \\
=\int_{\Omega} h^{\prime} u_{t t} d x+M^{\prime}\left(\|\nabla u(t)\|_{2}^{2}\right) 2\left(\nabla u_{t}, \nabla u\right)\left(\Delta u, u_{t t}\right) \\
\quad+M\left(\|\nabla u(t)\|_{2}^{2}\right)\left(\Delta u_{t}, u_{t t}\right)-(\rho+1) \int_{\Omega}|u|^{\rho} u_{t} u_{t t} d x
\end{aligned}
$$

Usando que $M$ é de classe $C^{1}$, temos

$$
\begin{aligned}
& M^{\prime}\left(\|\nabla u(t)\|_{2}^{2}\right) 2\left(\nabla u_{t}, \nabla u\right)\left(\Delta u, u_{t t}\right) \\
& \leq C_{4}\left\{\left\|u_{t t}(t)\right\|_{2}^{2}+\left\|\Delta u_{t}(t)\right\|_{2}^{2}\right\}
\end{aligned}
$$

$\mathrm{e}$

$$
M\left(\|\nabla u(t)\|_{2}^{2}\right)\left(\Delta u_{t}, u_{t t}\right) \leq C_{5}\left\{\left\|u_{t t}(t)\right\|_{2}^{2}+\left\|\Delta u_{t}(t)\right\|_{2}^{2}\right\}
$$

Novamente, usando a imersão $H_{0}^{2}(\Omega) \hookrightarrow H_{0}^{1}(\Omega) \hookrightarrow L^{2(\rho+1)}(\Omega)$, obtemos

$$
\begin{aligned}
(\rho+1) \int_{\Omega}|u|^{\rho} u_{t} u_{t t} d x & \leq(\rho+1)\|u(t)\|_{2(\rho+1)}^{\rho}\left\|u_{t}(t)\right\|_{2(\rho+1)}\left\|u_{t t}(t)\right\|_{2} \\
& \leq C_{6}\left\{\left\|u_{t t}(t)\right\|_{2}^{2}+\left\|\Delta u_{t}(t)\right\|_{2}^{2}\right\} .
\end{aligned}
$$

Substituindo (2.23)-(2.25) in (2.22), encontramos

$$
\frac{d}{d t}\left\{\left\|u_{t t}(t)\right\|_{2}^{2}+\left\|\Delta u_{t}(t)\right\|_{2}^{2}\right\} \leq\left\|h^{\prime}(t)\right\|_{2}^{2}+C_{7}\left\{\left\|u_{t t}(t)\right\|_{2}^{2}+\left\|\Delta u_{t}(t)\right\|_{2}^{2}\right\}
$$


Integrando de 0 até $t \leq T_{\max }$, obtemos

$$
\begin{aligned}
& \left\|u_{t t}(t)\right\|_{2}^{2}+\left\|\Delta u_{t}(t)\right\|_{2}^{2} \leq \int_{0}^{t}\left\|h^{\prime}(s)\right\|_{2}^{2} d s \\
& \quad+\left\|u_{t t}(0)\right\|_{2}^{2}+\left\|\Delta u_{t}(0)\right\|_{2}^{2}+C_{7} \int_{0}^{t}\left\{\left\|u_{t t}(s)\right\|_{2}^{2}+\left\|\Delta u_{t}(s)\right\|_{2}^{2}\right\} d s .
\end{aligned}
$$

Usando que $\left(u_{0}, u_{1}\right) \in D(A)$ e $h \in C^{1}\left([0, \infty) ; L^{2}(\Omega)\right)$ existe uma constante $C_{8}>0$ tal que

$$
\int_{0}^{t}\left\|h^{\prime}(s)\right\|_{2}^{2} d s+\left\|u_{t t}(0)\right\|_{2}^{2}+\left\|\Delta u_{t}(0)\right\|_{2}^{2} \leq C_{8}, \quad \forall t \in\left[0, T_{\max }\right)
$$

Do lema de Gronwall, obtemos

$$
\left\|u_{t t}(t)\right\|_{2}^{2}+\left\|\Delta u_{t}(t)\right\|_{2}^{2} \leq C_{9}, \quad \forall t \in\left[0, t_{\max }\right)
$$

Agora, como

$$
u_{t t}, M\left(\|\nabla u(t)\|_{2}^{2}\right) \Delta u,|u|^{\rho} u, u_{t} \in L^{\infty}\left(0, T_{\max } ; L^{2}(\Omega)\right)
$$

e

$$
\Delta^{2} u=u_{t t}-M\left(\|\nabla u(t)\|_{2}^{2}\right) \Delta u+|u|^{\rho} u+\beta u_{t},
$$

deduzimos que

$$
\left\|\Delta^{2} u\right\|_{2}=\left\|u_{t t}-M\left(\|\nabla u(t)\|_{2}^{2}\right) \Delta u+|u|^{\rho} u+\beta u_{t}\right\|_{2} \leq C_{10}
$$

para todo $t \in\left[0, T_{\max }\right)$.

Logo, de (2.28) e (2.29), resulta que

$$
\lim _{t \rightarrow T_{\max }}\left\{\left\|\Delta^{2} u(t)\right\|_{2}^{2}+\left\|\Delta u_{t}(t)\right\|_{2}^{2}\right\}<\infty
$$

Isto prova o Lema 2.1.3.

Do Lema 2.1.3 segue que $T_{\max }=+\infty$ e conseqüentemente $U=\left(u, u_{t}\right)$ existe globalmente.

Finalizando, mostraremos a unicidade: Suponhamos que (2.1) possui duas soluções, digamos $u$ e $v$, com mesmo dados iniciais em $t=0$. Então a diferença das duas soluções $w=u-v$ 
satisfaz

$$
\begin{aligned}
& w_{t t}+\Delta^{2} w-\left[M\left(\|\nabla u(t)\|_{2}^{2}\right) \Delta u-M\left(\|\nabla v\|_{2}^{2}\right) \Delta v\right] \\
& +\left[|u|^{\rho} u-|v|^{\rho} v\right]+\beta w_{t}=0 .
\end{aligned}
$$

Multiplicando (2.30) por $w_{t}$ e integrando sobre $\Omega$, obtemos

$$
\begin{aligned}
\frac{1}{2} \frac{d}{d}\{ & \left.\left\|w_{t}(t)\right\|_{2}^{2}+\|\Delta w(t)\|_{2}^{2}+M\left(\|\nabla u(t)\|_{2}^{2}\right)\|\nabla w(t)\|_{2}^{2}\right\} \\
& +\beta\left\|w_{t}(t)\right\|_{2}^{2}+\int_{\Omega}\left(|u|^{\rho} u-|v|^{\rho} v\right)(u-v) d x \\
= & M^{\prime}\left(\|\nabla u(t)\|_{2}^{2}\right)\left(\nabla u_{t}, \nabla u\right)\|\Delta w(t)\|_{2}^{2} \\
& +\left[M\left(\|\nabla u(t)\|_{2}^{2}\right)-M\left(\|v(t)\|_{2}^{2}\right)\right] \int_{\Omega} \Delta v w d x .
\end{aligned}
$$

Usando que $M$ é de classe $C^{1}$, temos

$$
M^{\prime}\left(\|\nabla u(t)\|_{2}^{2}\right)\left(\nabla u_{t}, \nabla u\right)\|\Delta w(t)\|_{2}^{2} \leq C_{11}\|\Delta w(t)\|_{2}^{2} .
$$

Pelo Teorema do Valor Médio e da imersão $H_{0}^{2}(\Omega) \hookrightarrow H_{0}^{1}(\Omega)$, obtemos

$$
\left[M\left(\|\nabla u(t)\|_{2}^{2}\right)-M\left(\|\nabla v(t)\|_{2}^{2}\right)\right] \int_{\Omega} \Delta v w d x \leq C_{12}\|\Delta w(t)\|_{2}^{2} .
$$

Substituindo (2.32) e (2.33) em (2.31) e integrando de 0 até $t$, resulta que

$$
\begin{aligned}
& \left\|w_{t}(t)\right\|_{2}^{2}+\|\Delta w(t)\|_{2}^{2}+M\left(\|\nabla u(t)\|_{2}^{2}\right)\|\nabla w(t)\|_{2}^{2} \\
& \quad+\beta \int_{0}^{t}\left\|w_{t}(s)\right\|_{2}^{2} d s+\int_{0}^{t} \int_{\Omega}\left(|u|^{\rho} u-|v|^{\rho} v\right)(u-v) d x d s \\
& \leq \int_{0}^{t}\left(\left\|w_{t}(s)\right\|_{2}^{2}+\|\Delta w(s)\|_{2}^{2}+M\left(\|\nabla u(s)\|_{2}^{2}\right)\|\nabla w(s)\|_{2}^{2}\right) d s .
\end{aligned}
$$

Usando que

$$
\beta \int_{0}^{t}\left\|w_{t}(s)\right\|_{2}^{2} d s+\int_{0}^{t} \int_{\Omega}\left(|u|^{\rho} u-|v|^{\rho} v\right)(u-v) d x d s \geq 0,
$$

segue do lema de Gronwall que

$$
\left\|w_{t}(t)\right\|_{2}^{2}+\|\Delta w(t)\|_{2}^{2} \leq 0, \forall t \geq 0
$$




\section{Portanto}

$$
\left\|w_{t}(t)\right\|_{2}=\|\Delta w(t)\|_{2}=0 \text {. }
$$

Isto prova a unicidade e completa a prova do Teorema 2.1.1.

\subsubsection{Existência de solução fraca}

A prova da existência de solução fraca em $E_{0}=H_{0}^{2}(\Omega) \times L^{2}(\Omega)$ é realizada via argumentos de densidade.

Teorema 2.1.2 Suponhamos que as hipóteses do Teorema 2.1.1 são válidas. Então, para qualquer

$$
\left(u_{0}, u_{1}\right) \in E_{0}=H_{0}^{2}(\Omega) \times L^{2}(\Omega)
$$

o problema (2.1) admite uma única solução fraca u na seguinte classe

$$
u \in C^{0}\left([0, \infty) ; H_{0}^{2}(\Omega)\right) \cap C^{1}\left([0, \infty) ; L^{2}(\Omega)\right) .
$$

\section{Demonstração:}

Seja $\left(u_{0}, u_{1}\right) \in H_{0}^{2}(\Omega) \times L^{2}(\Omega)$. Então, como

$$
H^{4}(\Omega) \cap H_{0}^{2}(\Omega) \times H_{0}^{2}(\Omega) \hookrightarrow H_{0}^{2}(\Omega) \times L^{2}(\Omega)
$$

é densamente imerso, existe uma sequência

$$
\left\{\left(u_{0}^{k}, u_{1}^{k}\right)\right\}_{k \in \mathbb{N}} \subset H^{4}(\Omega) \cap H_{0}^{2}(\Omega) \times H_{0}^{2}(\Omega)
$$

tal que

$$
\left(u_{0}^{k}, u_{1}^{k}\right) \longrightarrow\left(u_{0}, u_{1}\right) \text { em } H_{0}^{2}(\Omega) \times L^{2}(\Omega) .
$$

Do Teorema 2.1.1, para cada $\left(u_{0}^{k}, u_{1}^{k}\right) \in H^{4}(\Omega) \cap H_{0}^{2}(\Omega) \times H_{0}^{2}(\Omega)$ existe uma única solução global $U^{k}=\left(u^{k}, u_{t}^{k}\right)$ com

$$
\begin{gathered}
u^{k} \in L^{\infty}\left(0, T ; H^{4}(\Omega) \cap H_{0}^{2}(\Omega)\right), \\
u_{t}^{k} \in L^{\infty}\left(0, T ; H_{0}^{2}(\Omega)\right),
\end{gathered}
$$




$$
u_{t t}^{k} \in L^{\infty}\left(0, T ; L^{2}(\Omega)\right)
$$

para todo $T>0$ verificando

$$
\left\{\begin{array}{l}
u_{t t}^{k}+\Delta^{2} u^{k}-M\left(\left\|\nabla u^{k}\right\|_{2}^{2}\right) \Delta u^{k}+\beta u_{t}^{k}+\left|u^{k}\right|^{\rho} u^{k}=h \text { in } \Omega \times \mathbb{R}^{+} \\
\left(u^{k}(0), u_{t}^{k}(0)\right)=\left(u_{0}^{k}, u_{1}^{k}\right)
\end{array}\right.
$$

Multiplicando a equação de (2.36) por $u_{t}^{k}$, integrando sobre $\Omega$ e considerando argumentos análogos anteriormente usados, podemos deduzir que

$$
\begin{aligned}
& \left\|u_{t}^{k}(t)\right\|_{2}^{2}+\left\|\Delta u^{k}(t)\right\|_{2}^{2}+\widehat{M}\left(\left\|\nabla u^{k}(t)\right\|_{2}^{2}\right)+\frac{2}{\rho+2}\left\|u^{k}(t)\right\|_{\rho+2}^{\rho+2} \\
& \quad+\beta \int_{0}^{t}\left\|u_{t}^{k}(s)\right\|_{2}^{2} d s=\int_{0}^{t}\|h(s)\|_{2}^{2} d s \\
& \quad+\left\|u_{1}^{k}\right\|_{2}^{2}+\left\|\Delta u_{0}^{k}\right\|_{2}^{2}+\widehat{M}\left(\left\|\nabla u_{0}^{k}\right\|_{2}^{2}\right)+\frac{2}{\rho+2}\left\|u_{0}^{k}\right\|_{\rho+2}^{\rho+2} \leq C_{13} .
\end{aligned}
$$

onde $C_{13}$ uma constante positiva independente de $t \in[0, T]$ e $k \in \mathbb{N}$.

Agora, tomando $u^{k}$ e $u^{\mu}$ soluções de (2.36), temos

$$
\begin{aligned}
& \left\|\left(u_{t}^{k}-u_{t}^{\mu}\right)(t)\right\|_{2}^{2}+\left\|\Delta\left(u^{k}-u^{\mu}\right)(t)\right\|_{2}^{2} \\
& \quad \leq C_{13}\left(\left\|\left(u_{t}^{k}-u^{\mu}\right)(0)\right\|_{2}^{2}+\left\|\Delta\left(u^{k}-u^{\mu}\right)(0)\right\|_{2}^{2}\right) .
\end{aligned}
$$

Portanto, da estimativa (2.37) e (2.38), e considerando as convergências dadas em (2.35), obtemos uma função $u$ tal que

$$
\begin{array}{r}
u_{t}^{k} \stackrel{*}{\longrightarrow} u_{t} \text { em } L^{\infty}\left(0, T ; L^{2}(\Omega)\right), \\
u^{k} \stackrel{*}{\longrightarrow} u \text { em } L^{\infty}\left(0, T ; H_{0}^{2}(\Omega)\right), \\
u_{t}^{k} \longrightarrow u_{t} \text { em } C^{0}\left(0, T ; L^{2}(\Omega)\right), \\
u^{k} \longrightarrow u \text { em } C^{0}\left(0, T ; H_{0}^{2}(\Omega)\right), \\
\left|u^{k}\right|^{\rho} u^{k} \stackrel{*}{\longrightarrow}|u|^{\rho} u \text { em } L^{2}\left(0, T ; L^{2}(\Omega)\right) .
\end{array}
$$

Assim, podemos passar o limite em (2.36) obtendo

$$
u_{t t}+\Delta^{2} u-M\left(\|\nabla u\|_{2}^{2}\right) \Delta u+\beta u_{t}+|u|^{\rho} u=h \text { em } L_{l o c}^{2}\left(0, \infty ; H^{-2}(\Omega)\right)
$$


A unicidade de solução fraca requer um procedimento de regularização e pode ser obtida pelo método padrão de Visik-Ladyzhenkaya, cf. Lions e Magenes [28], Capítulo 3, Seção 8.2.2. Portanto, a prova do Teorema 2.1.2 fica completa. 


\section{Capítulo}

\section{Atrator Exponencial para o Problema com}

\section{Dissipação Linear}

Neste capítulo mostraremos a existência de conjuntos absorventes para o problema (2.1) nos espaços de Hilbert

$$
E_{0}=H_{0}^{2}(\Omega) \times L^{2}(\Omega)
$$

$\mathrm{e}$

$$
E_{1}=H^{4}(\Omega) \cap H_{0}^{2}(\Omega) \times H_{0}^{2}(\Omega),
$$

e a existência de um atrator global em $E_{0}$. O resultado principal é o Teorema 3.4.1, onde provamos a existência de um conjunto inércia que atrai exponencialmente todos os limitados de $E_{1}$ com respeito a norma de $E_{0}$.

Vamos assumir que a função $h$ é independente do tempo, isto é, consideramos apenas o caso autônomo

$$
h(t, x) \equiv h(x) \in L^{2}(\Omega), \quad \forall t \geq 0 .
$$

Definimos para todo $t \geq 0$, a aplicação

$$
\begin{aligned}
S(t): E_{0}=H_{0}^{2}(\Omega) \times L^{2}(\Omega) & \longrightarrow E_{0} \\
\left(u_{0}, u_{1}\right) & \longmapsto S(t)\left(u_{0}, u_{1}\right)=\left(u(t), u_{t}(t)\right),
\end{aligned}
$$


onde $u(t)$ é a única solução de (2.1) obtida pelo Teorema 2.1.2. Este operador não linear aplica $E_{0}$ nele mesmo e define um $C_{0}$-semigrupo não linear sobre $E_{0}$.

\subsection{Existência de um Conjunto Absorvente em $E_{0}$}

A existência de um conjunto absorvente em $E_{0}=H_{0}^{2}(\Omega) \times L^{2}(\Omega)$ é assegurada pelo seguinte Lema.

Lema 3.1.1 Sobre as hipóteses do Teorema 2.1.2, o semigrupo não linear $S(t): E_{0} \rightarrow E_{0}$ dado por

$$
S(t)\left(u_{0}, u_{1}\right)=\left(u(t), u_{t}(t)\right)
$$

possui um conjunto absorvente $B_{0}$ em $E_{0}$.

\section{Demonstração:}

Fixemos um conjunto limitado arbitrário $B \subset E_{0}$ e consideremos as soluções do problema (2.1) dadas por $\left(u(t), u_{t}(t)\right)=S(t)\left(u_{0}, u_{1}\right) \operatorname{com}\left(u_{0}, u_{1}\right) \in B$.

A energia do problema é dada por

$$
E(t)=\frac{1}{2}\left(\left\|u_{t}(t)\right\|_{2}^{2}+\|\Delta u(t)\|_{2}^{2}+\widehat{M}\left(\|\nabla u(t)\|_{2}^{2}\right)+\frac{2}{\rho+2}\|u(t)\|_{\rho+2}^{\rho+2}\right)-\int_{\Omega} h u d x
$$

que pode não majorar a norma de $E_{0}$. Definimos então o seguinte funcional energia modificado

$$
\widetilde{E}(t)=E(t)+\frac{1}{\lambda_{1}}\|h\|_{2}^{2}
$$

onde $\lambda_{1}$ denota o primeiro autovalor do operador bi-harmônico $\Delta^{2}$ em $H_{0}^{2}(\Omega)$. Sabemos que

$$
\|u\|_{2}^{2} \leq \lambda_{1}^{-1}\|\Delta u\|_{2}^{2}, \quad \forall u \in H_{0}^{2}(\Omega)
$$

e então

$$
\int_{\Omega} h(x) u(x, t) d x \leq \frac{1}{\lambda_{1}}\|h\|_{2}^{2}+\frac{1}{4}\|\Delta u(t)\|_{2}^{2}
$$

o que mostra que

$$
\widetilde{E}(t) \geq \frac{1}{4}\left(\left\|u_{t}(t)\right\|_{2}^{2}+\|\Delta u(t)\|_{2}^{2}\right)
$$


Multiplicando (2.1) por $u_{t}$ e integrando sobre $\Omega$ obtemos a seguinte identidade

$$
\frac{d}{d t} E(t)=-\beta\left\|u_{t}(t)\right\|_{2}^{2}
$$

e então, integrando de $t$ até $t+1$ resulta que

$$
0 \leq \beta \int_{t}^{t+1} \int_{\Omega}\left|u_{t}(x, s)\right|^{2} d x d s=E(t)-E(t+1) \equiv D(t)^{2}
$$

onde também fica definida a diferença $D(t)$.

Pelo Teorema do Valor Médio para integrais, existem $t_{1} \in\left[t, t+\frac{1}{4}\right]$ e $t_{2} \in\left[t+\frac{3}{4}, t+1\right]$ tais que

$$
\left\|u_{t}\left(t_{i}\right)\right\|_{2}^{2} \leq 4 \int_{t}^{t+1}\left\|u_{t}(s)\right\|_{2}^{2} d s=\frac{4}{\beta} D(t)^{2}, \quad i=1,2
$$

Prosseguindo, multiplicando a equação (2.1) por $u$ e integrando sobre $\left[t_{1}, t_{2}\right] \times \Omega$, temos

$$
\begin{gathered}
\int_{t_{1}}^{t_{2}}\left(\|\Delta u(s)\|_{2}^{2}+M\left(\|\nabla u(s)\|_{2}^{2}\right)\|\nabla u(s)\|_{2}^{2}+\|u(s)\|_{\rho+2}^{\rho+2}\right) d s \\
=\int_{t_{1}}^{t_{2}}\left\|u_{t}(s)\right\|_{2}^{2} d s-\left(\int_{\Omega} u_{t}\left(t_{1}\right) u\left(t_{1}\right) d x-\int_{\Omega} u_{t}\left(t_{2}\right) u\left(t_{2}\right) d x\right) \\
+\beta \int_{t_{1}}^{t_{2}} \int_{\Omega} u_{t} u d x d s+\int_{t_{1}}^{t_{2}} \int_{\Omega} h u d x d s .
\end{gathered}
$$

Somando $\int_{t_{1}}^{t_{2}}\left(\left\|u_{t}(s)\right\|_{2}^{2}-2 \int_{\Omega} h u d x\right) d s+\frac{2}{\lambda_{1}}\|h\|_{2}^{2}$ em ambos os lados da igualdade (3.6) e usando a definição de $\widetilde{E}(t)$, obtemos

$$
\begin{aligned}
\int_{t_{1}}^{t_{2}} \widetilde{E}(s) d s \leq & \int_{t_{1}}^{t_{2}}\left\|u_{t}(s)\right\|_{2}^{2} d s-\frac{1}{2}\left(\int_{\Omega} u_{t}\left(t_{1}\right) u\left(t_{1}\right) d x-\int_{\Omega} u_{t}\left(t_{2}\right) u\left(t_{2}\right) d x\right) \\
& +\frac{\beta}{2} \int_{t_{1}}^{t_{2}} \int_{\Omega} u_{t} u d x d s-\frac{1}{2} \int_{t_{1}}^{t_{2}} \int_{\Omega} h u d x d s+\frac{1}{\lambda_{1}}\|h\|_{2}^{2} .
\end{aligned}
$$

Vamos estimar o lado direito de (3.7).

De (3.4) temos

$$
\int_{t}^{t+1}\left\|u_{t}(s)\right\|_{2}^{2} d s \leq \frac{1}{\beta} D(t)^{2} .
$$


De (3.2), (3.4) e (3.5),

$$
\begin{aligned}
-\frac{1}{2}\left(\int_{\Omega} u_{t}\left(t_{1}\right) u\left(t_{1}\right)-u_{t}\left(t_{2}\right) u\left(t_{2}\right) d x\right) & \leq \max _{i=1,2}\left\{\left\|u_{t}\left(t_{i}\right)\right\|_{2}\left\|u\left(t_{i}\right)\right\|_{2}\right\} \\
& \leq \frac{2}{\beta^{1 / 2}} D(t) \frac{1}{\lambda_{1}^{1 / 2}} \sup _{t \leq s \leq t+1}\|\Delta u(s)\|_{2} .
\end{aligned}
$$

Analogamente,

$$
\begin{aligned}
\frac{\beta}{2} \int_{t_{1}}^{t_{2}} \int_{\Omega} u_{t} u d x d s & \leq \frac{\beta}{2} \sup _{t \leq s \leq t+1}\|u(t)\|_{2} \int_{t}^{t+1}\left\|u_{t}(s)\right\|_{2} d s \\
& \leq \frac{\beta^{1 / 2}}{2 \lambda_{1}^{1 / 2}} D(t) \sup _{t \leq s \leq t+1}\|\Delta u(s)\|_{2},
\end{aligned}
$$

$\mathrm{e}$

$$
-\frac{1}{2} \int_{t_{1}}^{t_{2}} \int_{\Omega} h u d x d s \leq \frac{1}{2 \lambda_{1}^{1 / 2}}\|h\|_{2} \sup _{t \leq s \leq t+1}\|\Delta u(s)\|_{2}
$$

Portanto, em vista de (3.8)-(3.11), temos de (3.7),

$$
\begin{aligned}
\int_{t_{1}}^{t_{2}} \widetilde{E}(s) d s \leq & \frac{1}{\beta} D(t)^{2}+\left(\frac{2}{\beta^{1 / 2} \lambda_{1}^{1 / 2}}+\frac{\beta^{1 / 2}}{2 \lambda_{1}^{1 / 2}}\right) D(t) \sup _{t \leq s \leq t+1}\|\Delta u(s)\|_{2} \\
& +\frac{1}{\lambda_{1}^{1 / 2}}\|h\|_{2} \sup _{t \leq s \leq t+1}\|\Delta u(s)\|_{2}+\frac{1}{\lambda_{1}}\|h\|_{2}^{2} .
\end{aligned}
$$

Aplicando a desigualdade de Young com $\varepsilon$ e notando que $\|\Delta u(t)\|_{2}^{2} \leq \widetilde{E}(t)$, podemos obter

$$
\int_{t_{1}}^{t_{2}} \widetilde{E}(s) d s \leq \varepsilon \sup _{t \leq s \leq t+1} \widetilde{E}(s)+C_{1}(\varepsilon) D(t)^{2}+C_{2}(\varepsilon)\|h\|_{2}^{2},
$$

onde $C_{i}(\varepsilon)>0, i=1,2$ são constantes que dependem apenas de $\beta, h, \varepsilon$.

Novamente, usando o Teorema do Valor Médio para integrais existe $t^{*} \in\left(t_{1}, t_{2}\right)$ tal que

$$
\widetilde{E}\left(t^{*}\right) \leq 2 \varepsilon \sup _{t \leq s \leq t+1} \widetilde{E}(s)+2 C_{1}(\varepsilon) D(t)^{2}+2 C_{2}(\varepsilon)\|h\|_{2}^{2}
$$


Como $E(t)-E(t+1)=\widetilde{E}(t)-\widetilde{E}(t+1)$ e $E$ é descrecente, de (3.4) vemos que

$$
\widetilde{E}(t+1) \leq \widetilde{E}\left(t^{*}\right) \quad \text { e } \quad \widetilde{E}(t)=\widetilde{E}(t+1)+D(t)^{2} .
$$

Portanto obtemos

$$
\widetilde{E}(t) \leq \widetilde{E}\left(t^{*}\right)+D(t)^{2}
$$

Combinando (3.14) e (3.15) resulta que

$$
\sup _{t \leq s \leq t+1} \widetilde{E}(s) \leq 2 \varepsilon \sup _{t \leq s \leq t+1} \widetilde{E}(s)+C_{3}(\varepsilon) D^{2}(t)+2 C_{2}(\varepsilon)\|h\|_{2}^{2}
$$

Escolhendo $\varepsilon=\frac{1}{4}$ obtemos de (3.16) que

$$
\sup _{t \leq s \leq t+1} \widetilde{E}(s) \leq 2 C_{3}(\varepsilon) D(t)^{2}+4 C_{2}(\varepsilon)\|h\|_{2}^{2}
$$

Pela definição de $D(t)^{2}$ obtemos de (3.17)

$$
\sup _{t \leq s \leq t+1} \widetilde{E}(s) \leq 2 C_{3}(\varepsilon)(\widetilde{E}(t)-\widetilde{E}(t+1))+4 C_{2}(\varepsilon)\|h\|_{2}^{2}
$$

Aplicando Lema de Nakao (Lema 1.3.1) em (3.18) com $k_{0}=2 C_{3}(\varepsilon), k_{1}=4 C_{2}(\varepsilon)\|h\|_{2}^{2}$ e $\phi(s)=\widetilde{E}(s)$, resulta

$$
\widetilde{E}(s) \leq \sup _{0 \leq s \leq 1} \widetilde{E}(s)\left(\frac{1+2 C_{3}(\varepsilon)}{2 C_{3}(\varepsilon)}\right) e^{-\theta t}+4 C_{2}(\varepsilon)\|h\|_{2}^{2}, \quad 0 \leq t \leq T
$$

onde $\theta=\ln \left(\frac{1+2 C_{3}(\varepsilon)}{2 C_{3}(\varepsilon)}\right)$.

Em vista de (3.3)

$$
\left\|\left(u(t), u_{t}(t)\right)\right\|_{E_{0}}^{2} \leq C_{0} e^{-\theta t}+C_{0}\|h\|_{2}^{2}, \quad t \geq 0
$$


onde $C_{0}$ é uma constante que não depende de $t$. Então existe $t_{B}>0$ tal que

$$
\left\|\left(u(t), u_{t}(t)\right)\right\|_{E_{0}}^{2} \leq 2 C_{0}\|h\|_{2}^{2}, \quad t \geq t_{B}
$$

Portanto, escrevendo $r_{0}=2 C_{0}\|h\|_{2}^{2}$, segue que, a bola fechada $B_{0}=\bar{B}\left(0, r_{0}\right)$ de $E_{0}$ é um conjunto absorvente para $S(t)$. Isto completa a prova do Lema 3.1.1.

Assim, para provar que o problema (2.1) admite um atrator global, resta provar a propriedade de compacidade assintótica.

\subsection{Atrator Global em $E_{0}$}

Prosseguindo, mostraremos que $S(t)$ possui a propriedade de compacidade assintótica (ii) do Teorema 1.3.1. Para isto aplicaremos o Lema 1.3.2, com auxílio do Lema de Nakao.

Lema 3.2.1 Sobre as hipóteses do Teorema 2.1.1, o $C_{0}$-semigrupo não linear $\{S(t)\}_{t \geq 0}$ associado a problema (2.1) é compactamente assintótico em $E_{0}=H_{0}^{2}(\Omega) \times L^{2}(\Omega)$.

\section{Demonstração:}

Dados $\left(u_{0}, u_{1}\right),\left(v_{0}, v_{1}\right)$ pertencentes a um subconjunto $B$ limitado de $E_{0}$, sejam $u, v$ as correspondentes soluções do problema (2.1). Então do Teorema 2.1.2, $w=u-v$ é solução fraca para o seguinte problema

$$
\left\{\begin{array}{l}
w_{t t}+\Delta^{2} w+\beta w_{t}=M\left(\|\nabla u\|_{2}^{2}\right) \Delta u-M\left(\|\nabla v\|_{2}^{2}\right) \Delta v+\left(|u|^{\rho} u-|v|^{\rho} v\right) \quad \text { em } \quad \Omega \times \mathbb{R}^{+} \\
w=\frac{\partial u}{\partial v}=0 \quad \text { sobre } \quad \Gamma \times \mathbb{R}^{+}, \\
w(\cdot, 0)=u_{0}-v_{0}, \quad w_{t}(\cdot, 0)=u_{1}-v_{1} \quad \text { em } \quad \Omega .
\end{array}\right.
$$

Definimos o funcional

$$
E_{w}(t)=\left\|w_{t}(t)\right\|_{2}^{2}+\|\Delta w(t)\|_{2}^{2}+M\left(\|\nabla u(t)\|_{2}^{2}\right)\|\nabla w(t)\|_{2}^{2},
$$


Multiplicando a equação (3.20) por $w_{t}$ e integrando sobre $\Omega$, obtemos

$$
\begin{gathered}
\frac{1}{2} \frac{d}{d t} E_{w}(t)+\beta\left\|w_{t}(t)\right\|_{2}^{2}=M^{\prime}\left(\|\nabla u(t)\|_{2}^{2}\right) \int_{\Omega} u_{t}(t) \Delta u(t) d x\|\nabla w(t)\|_{2}^{2} \\
+\left(M\left(\|\nabla u(t)\|_{2}^{2}\right)-M\left(\|\nabla v(t)\|_{2}^{2}\right)\right) \int_{\Omega} \Delta v(t) w_{t}(t) d x \\
-\int_{\Omega}(f(u(t))-f(v(t))) w_{t}(t) d x .
\end{gathered}
$$

Agora vamos estimar o lado direito de (3.21). Nas desigualdades a seguir, usaremos o fato de que as soluções $u(t), v(t)$, com dados iniciais em $B$, são uniformemente limitadas na norma de $E_{0}$ por uma constante dependente de $B$, conforme as estimativas no Capítulo 2.

Pela continuidade de $M^{\prime}$ temos

$$
M^{\prime}\left(\|\nabla u(t)\|_{2}^{2}\right) \int_{\Omega} u_{t}(t) \Delta u(t) d x\|\nabla w(t)\|_{2}^{2} \leq C_{0}(B)\|\nabla w(t)\|_{2}^{2}
$$

Por outro lado, como $M\left(a^{2}\right)-M\left(b^{2}\right) \leq M^{\prime}\left(\sup \left\{a^{2}, b^{2}\right\}\right)(|a|+|b|)|a-b|$, e usando a desigualdade de Young $\operatorname{com} \varepsilon$ vemos que

$$
\begin{gathered}
\left(M\left(\|\nabla u(t)\|_{2}^{2}\right)-M\left(\|\nabla v(t)\|_{2}^{2}\right)\right) \int_{\Omega} \Delta v(t) w_{t}(t) d x \\
\leq C_{1}(B)\|\nabla w(t)\|_{2}\left\|w_{t}(t)\right\|_{2} \\
\leq C_{2}(B, \varepsilon)\|\nabla w(t)\|_{2}^{2}+\varepsilon\left\|w_{t}(t)\right\|_{2}^{2} .
\end{gathered}
$$

Aplicando a desigualdade de Hölder com $\frac{\rho}{2(\rho+1)}+\frac{1}{2(\rho+1)}+\frac{1}{2}=1$, inferimos que

$$
\begin{aligned}
\int_{\Omega}\left(f(u(t))-f(v(t)) w_{t}(t) d x\right. & \leq(\rho+1) \int_{\Omega} \sup \left\{|u|^{\rho},|v|^{\rho}\right\} w(t) w_{t}(t) d x \\
& \leq C_{3}(B)\left(\|u(t)\|_{2(\rho+1)}^{\rho}+\|v(t)\|_{2(\rho+1)}^{\rho}\right)\|w(t)\|_{2(\rho+1)}\left\|w_{t}(t)\right\|_{2} \\
& \leq C_{4}(B)\|\nabla w(t)\|_{2}\left\|w_{t}(t)\right\|_{2} \\
& \leq C_{5}(B, \varepsilon)\|\nabla w(t)\|_{2}^{2}+\varepsilon\left\|w_{t}(t)\right\|_{2}^{2} .
\end{aligned}
$$

Escolhendo $\varepsilon=\frac{\beta}{4}$ e substituindo (3.22)-(3.24) em (3.21) obtemos

$$
\frac{d}{d t} E_{w}(t)+\beta\left\|w_{t}(t)\right\|_{2}^{2} \leq C_{B}\|\nabla w(t)\|_{2}^{2}
$$


onde $C_{B}$ é uma constante que depende de $B$ mas não de $t$. No que segue, para simplificar usaremos $C_{B}$ para indicar todas as constantes que dependem de $B$.

Então integrando (3.25) de $t$ até $t+1$, obtemos

$$
\beta \int_{t}^{t+1}\left\|w_{t}(s)\right\|_{2}^{2} d s \leq E_{w}(t)-E_{w}(t+1)+C_{B} \int_{t}^{t+1}\|\nabla w(s)\|_{2}^{2} d s \equiv F(t)^{2}
$$

A função $F(t)$ irá desempenhar um papel semelhante ao que desempenhou a funcão $D(t)$ definida em (3.4).

De (3.26) resulta que

$$
\int_{t}^{t+1}\left\|w_{t}(s)\right\|_{2}^{2} d s \leq \frac{1}{\beta} F(t)^{2}
$$

Pelo Teorema do Valor Médio para integrais existem $t_{1} \in\left[t, t+\frac{1}{4}\right]$ e $t_{2} \in\left[t+\frac{3}{4}, t+1\right]$ tal que

$$
\left\|w_{t}\left(t_{i}\right)\right\|_{2}^{2} \leq \frac{4}{\beta} F^{2}(t), \quad i=1,2
$$

Agora, multiplicando a equação em (3.20) por $w$ e integrando sobre $\Omega$, obtemos

$$
\begin{gathered}
\|\Delta w(t)\|_{2}^{2}+M\left(\|\nabla u(t)\|_{2}^{2}\right)\|\nabla w(t)\|_{2}^{2}+\int_{\Omega}(f(u(t))-f(v(t)) w(t) d x \\
=\frac{d}{d t} \int_{\Omega} w_{t}(t) w(t) d x+\left\|w_{t}(t)\right\|_{2}^{2}+\beta \int_{\Omega} w_{t}(t) w(t) d x \\
\quad+\left(M\left(\|\nabla u(t)\|_{2}^{2}\right)-M\left(\|\nabla v(t)\|_{2}^{2}\right)\right) \int_{\Omega} \Delta v(t) w(t) d x .
\end{gathered}
$$

Integrando (3.29) de $t_{1}$ até $t_{2}$ e então usando (3.27), (3.28) e a monotocidade de $f(s)=|s|^{\rho_{s}}$, concluimos que

$$
\begin{aligned}
& \int_{t_{1}}^{t_{2}}\left(\|\Delta w(s)\|_{2}^{2}+M\left(\|\nabla u(s)\|_{2}^{2}\right)\|\nabla w(s)\|_{2}^{2}\right) d s \\
& \quad \leq \frac{1}{\beta} F(t)^{2}+\frac{4}{\lambda_{1}^{1 / 2} \beta^{1 / 2}} F(t) \sup _{t \leq s \leq t+1}\|\Delta w(s)\|_{2} \\
& \quad+\frac{\beta}{\lambda_{1}^{1 / 2}} F(t) \sup _{t \leq s \leq t+1}\|\Delta w(s)\|_{2}+\int_{t_{1}}^{t_{2}}\left(M\left(\|\nabla u(s)\|_{2}^{2}\right)-M\left(\nabla v(s) \|_{2}^{2}\right)\right) \int_{\Omega} \Delta v(s) w(s) d x d s .
\end{aligned}
$$


Novamente, usando que $M$ é de classe $C^{1}$ obtemos

$$
\int_{t_{1}}^{t_{2}}\left(M\left(\|\nabla u(s)\|_{2}^{2}\right)-M\left(\|\nabla v(s)\|_{2}^{2}\right)\right) \int_{\Omega} \Delta v(s) w(s) d x d s \leq C_{6} \int_{t_{1}}^{t_{2}}\|\nabla w(s)\|_{2}^{2} d s .
$$

Substituindo (3.31) em (3.30) resulta que

$$
\begin{aligned}
& \int_{t_{1}}^{t_{2}}\left(\|\Delta w(s)\|_{2}^{2}+M\left(\|\nabla u(s)\|_{2}^{2}\right)\|\nabla w(s)\|_{2}^{2}\right) d s \\
& \leq \frac{1}{\beta} F(t)^{2}+\frac{4}{\lambda_{1}^{1 / 2} \beta^{1 / 2}} F(t) \sup _{t \leq s \leq t+1}\|\Delta w(s)\|_{2} \\
& \quad+\frac{\beta}{\lambda_{1}^{1 / 2}} F(t) \sup _{t \leq s \leq t+1}\|\Delta w(s)\|_{2}+C_{6} \int_{t_{1}}^{t_{2}}\|\nabla w(s)\|_{2}^{2} d s
\end{aligned}
$$

Somando $\int_{t_{1}}^{t_{2}}\left\|w_{t}(s)\right\|^{2} d s$ em ambos os lados de (3.32), usando (3.27) e a definição de $E_{w}$ enconramos

$$
\begin{aligned}
\int_{t_{1}}^{t_{2}} E_{w}(s) d s \leq & \frac{2}{\beta} F(t)^{2}+\frac{4}{\lambda_{1}^{1 / 2} \beta^{1 / 2}} F(t) \sup _{t \leq s \leq t+1} E_{w}^{1 / 2}(s) \\
& +\frac{\beta}{\lambda_{1}^{1 / 2}} F(t) \sup _{t \leq s \leq t+1} E_{w}^{1 / 2}(s)+C_{6} \int_{t_{1}}^{t_{2}}\|\nabla w(s)\|_{2}^{2} d s .
\end{aligned}
$$

Usando a desigualdade de Young com $\varepsilon$ obtemos

$$
\int_{t_{1}}^{t_{2}} E_{w}(s) d s \leq C_{7}(\varepsilon) F^{2}(t)+\varepsilon \sup _{t \leq s \leq t+1} E_{w}(s)+C_{6} \int_{t_{1}}^{t_{2}}\|\nabla w(s)\|_{2}^{2} d s .
$$

Pelo Teorema do Valor Médio, existe $t^{*} \in\left[t_{1}, t_{2}\right]$ tal que

$$
E_{w}\left(t^{*}\right) \leq 2 C_{7}(\varepsilon) F^{2}(t)+2 \varepsilon \sup _{t \leq s \leq t+1} E_{w}(s)+2 C_{6} \int_{t_{1}}^{t_{2}}\|\nabla w(s)\|_{2}^{2} d s
$$

Por outro lado, de (3.26),

$$
E_{w}(t) \leq E_{w}(t+1)+F(t)^{2},
$$

e assim

$$
\sup _{t \leq s \leq t+1} E_{w}(s) \leq E_{w}\left(t^{*}\right)+F^{2}(t)+C_{B} \int_{t}^{t+1}\|\nabla w(s)\|_{2}^{2} d s .
$$


Assim, tomando $\varepsilon=\frac{1}{4}$ e substituindo (3.35) em (3.36) obtemos

$$
\sup _{t \leq s \leq t+1} E_{w}(s) \leq C_{8} F^{2}(t)+C_{B} \int_{t}^{t+1}\|\nabla w(s)\|_{2}^{2} d s
$$

Substituindo (3.26) em (3.37) temos

$$
\sup _{t \leq s \leq t+1} E_{w}(s) \leq C_{9}\left(E_{w}(t)-E_{w}(t+1)\right)+C_{B} \sup _{0 \leq \sigma \leq T} \int_{\sigma}^{\sigma+1}\|\nabla w(s)\|_{2}^{2} d s
$$

Usando o Lema Lema 1.3.1 (Nakao) obtemos

$$
E_{w}(t) \leq C_{10} e^{-\theta t}+C_{B} \sup _{0 \leq \sigma \leq T} \int_{\sigma}^{\sigma+1}\|\nabla w(s)\|_{2}^{2} d s, \quad 0 \leq t \leq T,
$$

e da definição de $E_{w}$,

$$
\|w(t)\|_{E_{0}} \leq C_{10} e^{-\frac{\theta}{2} t}+C_{B} \sup _{0 \leq \sigma \leq T}\left(\int_{\sigma}^{\sigma+1}\|\nabla w(s)\|_{2}^{2} d s\right)^{\frac{1}{2}}, \quad 0 \leq t \leq T .
$$

Dado $\varepsilon>0$, podemos escolher $T$ suficientemente grande tal que

$$
C_{10} e^{-\frac{\theta}{2} T}<\varepsilon
$$

Fixado este $T$, definimos $\phi_{T}: B_{0} \times B_{0} \rightarrow \mathbb{R}$ por

$$
\phi_{T}\left(\left(u_{0}, u_{1}\right),\left(v_{0}, v_{1}\right)\right)=C_{B} \sup _{0 \leq \sigma \leq T}\left(\int_{\sigma}^{\sigma+1}\|\nabla u(s)-\nabla v(s)\|_{2}^{2} d s\right)^{\frac{1}{2}}
$$

Assim, de (3.39), (3.40) e (3.41) encontramos

$$
\left\|S(T)\left(u_{0}, u_{1}\right)-S(T)\left(v_{0}, v_{1}\right)\right\|_{E_{0}} \leq \varepsilon+\phi_{T}\left(\left(u_{0}, u_{1}\right),\left(v_{0}, v_{1}\right)\right),
$$

para todo $\left(u_{0}, u_{1}\right),\left(v_{0}, v_{1}\right) \in B_{0}$. 
Para aplicar o Lema 1.3.2 resta mostrar que $\phi$ é uma função contrativa, isto é, para qualquer sequência $\left\{\left(u_{0}^{n}, u_{1}^{n}\right)\right\}$ de $B_{0}$, existe uma subsequência $\left\{\left(u_{0}^{n_{k}}, u_{1}^{n_{k}}\right)\right\}$ tal que

$$
\lim _{k \rightarrow \infty} \lim _{l \rightarrow \infty} \phi_{T}\left(\left(u_{0}^{n_{k}}, u_{1}^{n_{k}}\right),\left(u_{0}^{n_{l}}, u_{1}^{n_{l}}\right)\right)=0 .
$$

De fato, como $B_{0}$ é limitado e invariante sob $S(t)$, as soluções $\left\{\left(u^{n}(t), u_{t}^{n}(t)\right)\right\}$ são uniformemente limitados em $E_{0}$. Portanto

$$
u^{n} \text { é limitado em } C\left([0, \infty), H_{0}^{2}(\Omega)\right) \cap C^{1}\left([0, \infty) ; L^{2}(\Omega)\right)
$$

Por outro lado, a imersão compacta de $H_{0}^{2}(\Omega)$ em $H_{0}^{1}(\Omega)$ implica que a imersão

$$
C\left([0, T], H_{0}^{2}(\Omega)\right) \cap C^{1}\left([0, T] ; L^{2}(\Omega)\right) \hookrightarrow C\left([0, T] ; H_{0}^{1}(\Omega)\right)
$$

é compacta para cada $T>0$. (Simon [46]).

Assim $u^{n}$ possui uma subsequência $u^{n_{k}}$ que converge forte em $C\left([0, T] ; H_{0}^{1}(\Omega)\right)$, e portanto temos que o limite (3.43) é satisfeito .

Vimos que, do Lema 3.1.1 e Lema 3.2.1 que o semigrupo $\{S(t)\}_{t \geq 0}$ possui um conjunto absorvente em $E_{0}$ e $S(t)$ é compactamente assintótico em $E_{0}$, respectivamente. Então segue do Teorema 1.3.1 o seguinte resultado.

Teorema 3.2.1 Sob as hipóteses do Teorema 2.1.1, o $C_{0}$-semigrupo $\{S(t)\}_{t \geq 0}$ correspondente ao problema (2.1) possui um atrator global $\mathscr{A}$ em $E_{0}=H_{0}^{2}(\Omega) \times L^{2}(\Omega)$.

\subsection{Existência de um Conjunto Absorvente em $E_{1}$}

Nesta seção provaremos que o semigrupo $\{S(t)\}_{t \geq 0}$ também possui um conjunto absorvente em $E_{1}=H^{4}(\Omega) \cap H_{0}^{2}(\Omega) \times H_{0}^{2}(\Omega)$. Utilizaremos a técnica de multiplicadores, com argumentos semelhantes aos usados por Biazutti e Crippa [10]. O resultado de existência de um conjunto absorvente em $E_{1}$ é o seguinte.

Lema 3.3.1 Sobre as hipóteses do Teorema 2.1.1, o semigrupo $\{S(t)\}_{t \geq 0}$ associado com o problema 2.1 possui um conjunto absorvente em $E_{1}=H^{4}(\Omega) \cap H_{0}^{2}(\Omega) \times H_{0}^{2}(\Omega)$. 


\section{Demonstração:}

Suponhamos que os dados iniciais $\left(u_{0}, u_{1}\right)$ pertencentes a um subconjunto limitado $B$ de $E_{1}$ e $\left(u(t), u_{t}(t)\right)=S(t)\left(u_{0}, u_{1}\right)$. Seja $v=u_{t}+\varepsilon u \operatorname{com} \varepsilon \leq \frac{2 \beta}{5}$. Então podemos reescrever a equação (2.1) da seguinte forma

$$
v_{t}+\Delta^{2} u+(\beta-\varepsilon) v-\varepsilon(\beta-\varepsilon) u-M\left(\|\nabla u(t)\|_{2}^{2}\right) \Delta u+|u|^{\rho} u=h(x) .
$$

Consideremos o funcional $E_{v}$ associado a equação (3.44)

$$
E_{v}(t)=\|\Delta v(t)\|_{2}^{2}+\left\|\Delta^{2} u(t)-h\right\|_{2}^{2}+M\left(\|\nabla u(t)\|_{2}^{2}\right)\|\nabla(\Delta u(t))\|_{2}^{2}
$$

Sabemos que para mostrar que $\{S(t)\}_{t \geq 0}$ possui um conjunto absorvente em $E_{1}$ precisamos mostrar que existe $t_{1}(B)$ tal que

$$
\left\|\Delta^{2} u(t)\right\|_{2}^{2}+\|\Delta u(t)\|_{2}^{2}+\left\|\Delta u_{t}(t)\right\|_{2}^{2} \leq C \quad \text { para todo } \quad t \geq t_{1}(B)
$$

onde $C$ é uma constante que não depende dos dados iniciais. É fácil ver que isto fica provado se mostrarmos que o funcional $E_{v}$ satisfaz a seguinte desigualdade

$$
\frac{d}{d t} E_{v}(t)+C_{0} E_{v}(t) \leq C_{1}
$$

onde $C_{0}$ e $C_{1}$ são constantes que não dependem dos dados iniciais. Assim, no que segue, nosso objetivo é mostar que $E_{v}$ satifaz a desigualdade (3.46). Em ordem, pela regularidade (2.5) e usando argumentos de densidade, multiplicando (3.44) por $\Delta^{2} v$ e integrando sobre $\Omega$ obtemos

$$
\begin{aligned}
\frac{1}{2} \frac{d}{d t} & \left\{\|\Delta v(t)\|_{2}^{2}+\left\|\Delta^{2} u(t)\right\|_{2}^{2}-2 \int_{\Omega} h \Delta^{2} u d x\right\}+(\beta-\varepsilon)\|\Delta v(t)\|_{2}^{2} \\
& +\varepsilon\left\|\Delta^{2} u(t)\right\|_{2}^{2}-\varepsilon \int_{\Omega} h \Delta^{2} u d x=\varepsilon(\beta-\varepsilon) \int_{\Omega} u \Delta^{2} v d x \\
& -M\left(\|\nabla u(t)\|_{2}^{2}\right) \int_{\Omega} \Delta u \Delta^{2} v d x-\int_{\Omega}|u|^{\rho} u \Delta^{2} v d x
\end{aligned}
$$

Considerando que 


$$
\begin{aligned}
-M & \left(\|\nabla u(t)\|_{2}^{2}\right) \int_{\Omega} \Delta u \Delta^{2} v d x=-M\left(\|\nabla u(t)\|_{2}^{2}\right) \int_{\Omega} \Delta u \Delta^{2}\left(u_{t}+\varepsilon u\right) d x \\
= & -M\left(\|\nabla u(t)\|_{2}^{2}\right) \int_{\Omega} \Delta u \Delta^{2} u_{t} d x-\varepsilon M\left(\|\nabla u(t)\|_{2}^{2}\right) \int_{\Omega} \Delta u \Delta^{2} u d x \\
= & \frac{1}{2} \frac{d}{d t}\left(M\left(\|\nabla u(t)\|_{2}^{2}\right)\|\nabla(\Delta u(t))\|_{2}^{2}\right)-M^{\prime} \int_{\Omega} \nabla u \nabla u_{t} d x \int_{\Omega} \Delta u \Delta^{2} u d x \\
& +\varepsilon M\left(\|\nabla u(t)\|^{2}\right)\|\nabla(\Delta u(t))\|_{2}^{2}
\end{aligned}
$$

e, além disso,

$$
\begin{aligned}
\frac{d}{d t}\left\|\Delta^{2} u(t)-h\right\|_{2}^{2} & =\frac{d}{d t}\left(\left\|\Delta^{2} u(t)\right\|_{2}^{2}-2 \int_{\Omega} h \Delta^{2} u d x+\|h\|_{2}^{2}\right) \\
& =\frac{d}{d t}\left(\left\|\Delta^{2} u(t)\right\|_{2}^{2}-2 \int_{\Omega} h \Delta^{2} u d x\right)
\end{aligned}
$$

pois $h=h(x)$ é independente de $t$.

Assim, substituindo (3.48) e (3.49) em (3.47) obtemos

$$
\begin{gathered}
\frac{1}{2} \frac{d}{d t} E_{v}(t)+(\beta-\varepsilon)\|\Delta v(t)\|_{2}^{2}+\frac{\varepsilon}{2}\left\|\Delta^{2} u(t)-h\right\|_{2}^{2}+\varepsilon M\left(\|\nabla u(t)\|_{2}^{2}\right)\|\nabla(\Delta u(t))\|_{2}^{2} \\
\quad+\frac{\varepsilon}{2}\left\|\Delta^{2} u(t)\right\|_{2}^{2}=\frac{\varepsilon}{2}\|h\|_{2}^{2}+\varepsilon(\beta-\varepsilon) \int_{\Omega} u \Delta^{2} v d x \\
+M^{\prime}\left(\|\nabla u(t)\|_{2}^{2}\right) \int_{\Omega}(-\Delta u) u_{t} d x \int_{\Omega} \Delta u \Delta^{2} u d x-\int_{\Omega}|u|^{\rho} u \Delta^{2} v d x .
\end{gathered}
$$

Pela desigualdade de Young temos que

$$
\begin{aligned}
\varepsilon(\beta-\varepsilon) \int_{\Omega} u \Delta^{2} v d x & =-\varepsilon(\beta-\varepsilon) \int_{\Omega} \Delta^{2} u v d x \\
& \leq \frac{\varepsilon}{4}\left\|\Delta^{2} u(t)\right\|_{2}^{2}+\varepsilon(\beta-\varepsilon)^{2}\|v(t)\|_{2}^{2}
\end{aligned}
$$

Usando a continuidade de $M^{\prime}$ e a desigualdade de Young com $\varepsilon$ obtemos

$$
\begin{aligned}
& M^{\prime}\left(\|\nabla u(t)\|_{2}^{2}\right) \int_{\Omega}(-\Delta u) u_{t} d x \int_{\Omega} \Delta u \Delta^{2} u d x \\
& \quad \leq\left(M^{\prime}\left(\|\nabla u(t)\|_{2}^{2}\right)\|\Delta u(t)\|_{2}\left\|u_{t}(t)\right\|_{2}\|\Delta u(t)\|_{2}\right)\left\|\Delta^{2} u(t)\right\|_{2} \\
& \quad \leq \frac{1}{\varepsilon}\left(M^{\prime}\left(\|\nabla u(t)\|_{2}^{2}\right)\|\Delta u(t)\|_{2}\left\|u_{t}(t)\right\|_{2}\|\Delta u(t)\|_{2}\right)^{2}+\frac{\varepsilon}{4}\left\|\Delta^{2} u(t)\right\|_{2}^{2} .
\end{aligned}
$$


Também pela desigualdade de Young $\operatorname{com} \varepsilon$ vemos que

$$
\begin{aligned}
-\int_{\Omega}|u|^{\rho} u \Delta^{2} v d x & =-\int_{\Omega} \Delta\left(|u|^{\rho} u\right) \Delta v d x \\
& \leq \frac{1}{4 \varepsilon}\left\|\Delta\left(|u|^{\rho} u\right)\right\|_{2}^{2}+\varepsilon\|\Delta v(t)\|_{2}^{2} .
\end{aligned}
$$

Substituindo (3.51) - (3.53) em (3.50) e usando que $\varepsilon \leq \frac{2 \beta}{5}$ obtemos

$$
\begin{gathered}
\frac{1}{2} \frac{d}{d t} E_{v}(t)+\frac{\varepsilon}{2} E_{v}(t) \leq \frac{\varepsilon}{2}\|h\|_{2}^{2}+\varepsilon(\beta-\varepsilon)^{2}\|v(t)\|_{2}^{2}+\frac{1}{4 \varepsilon}\|v(t)\|_{2}^{2} \\
+\frac{1}{\varepsilon}\left(M^{\prime}\left(\|\nabla u(t)\|_{2}^{2}\right)\|\Delta u(t)\|_{2}\left\|u_{t}(t)\right\|_{2}\|\Delta u(t)\|_{2}\right)^{2}
\end{gathered}
$$

Como $\left(u_{0}, u_{1}\right)$ pertencente a subconjunto limitado $B$ de $E_{1}$, então $B$ é também limitado em $E_{0}=H_{0}^{2}(\Omega) \times L^{2}(\Omega)$. Assim, do Lema 3.1.1 existe $t_{0}(B)$ tal que para todo $t \geq t_{0}(B)$

$$
\varepsilon(\beta-\varepsilon)^{2}\|v(t)\|_{2}^{2}+\frac{1}{4 \varepsilon}\|v(t)\|_{2}^{2}+\frac{1}{\varepsilon}\left(M^{\prime}\left(\|\nabla u(t)\|_{2}^{2}\right)\|\Delta u(t)\|_{2}\left\|u_{t}(t)\right\|_{2}\|\Delta u(t)\|_{2}\right)^{2} \leq \frac{C_{\mu_{0}}}{2}
$$

onde $C_{\mu_{0}}$ é uma constante positiva que depende do raio $\mu_{0}$ da bola absorvente em $E_{0}$. Retornando a (3.54) resulta que

$$
\frac{d}{d t} E_{v}(t)+\varepsilon E_{v}(t) \leq \varepsilon\|h\|_{2}^{2}+C_{\mu_{0}} \quad \text { para todo } \quad t \geq t_{0}(B)
$$

Assim, multiplicando (3.55) por $e^{\varepsilon t}$ e, em seguida, integrando de $t_{0}(B)$ até $t$ obtemos

$$
E_{v}(t) \leq E_{v}\left(t_{0}(B)\right) e^{-\varepsilon\left(t-t_{0}(B)\right)}+\left(\varepsilon\|h\|_{2}^{2}+C_{\mu_{0}}\right)\left(1-e^{-\varepsilon\left(t-t_{0}(B)\right)}\right) .
$$

Considerando o limite em (3.56) $\operatorname{com} t \rightarrow \infty$, existe $t_{1}(B)$ tal que

$$
\left\|\left(u(t), u_{t}(t)\right)\right\|_{E_{1}}^{2}=\left\|\Delta u_{t}(t)\right\|_{2}^{2}+\|\Delta u(t)\|_{2}^{2}+\left\|\Delta^{2} u(t)\right\|_{2}^{2} \leq \mu_{1}^{2} \quad \text { para todo } t \geq t_{1}(B),
$$

onde $\mu_{1}^{2}=\left(\varepsilon\|h\|_{2}^{2}+C_{\mu_{0}}\right)$. Portanto, a bola fechada $B_{1}=\bar{B}\left(0, \mu_{1}\right)$ de $E_{1}$ é um conjunto absorvente para o semigrupo $\{S(t)\}_{t \geq 0}$. Isto conclui a prova do Lema 3.3.1. 


\subsection{Atrator Exponencial (Conjunto Inércia)}

O objetivo principal desta seção é provar que o sistema dinâmico associado com o problema (2.1) possui um atrator exponencial sobre um subconjunto compacto invariante $X$ de $E_{1}$ com respeito a norma de $E_{0}$.

Para o subconjunto compacto invariante de $E_{0}$ onde vamos trabalhar escolhamos

$$
X=\varlimsup_{\bigcup_{t \geq t_{2}} S(t)\left(B_{0} \cap B_{1}\right)}^{E_{0}}
$$

onde $B_{0}$ e $B_{1}$ são, respectivamente, conjuntos absorventes em $E_{0}$ e $E_{1}$, e $t_{2}$ é suficientemente grande. Assim $X$ é invariante por $S(t)$ e compacto em $E_{0}$.

O resultado principal desta seção pode ser enunciado da seguinte maneira.

Teorema 3.4.1 Nas condições do Teorema 2.1.1, o sistema dinâmico associado ao problema (2.1) possui um atrator exponencial $\mathscr{M} \subset X$.

\section{Demonstração:}

Seja $\left\{\lambda_{n}\right\}_{n \geq 1}$ a sequência de autovalores do operador bi-harmônico $(-\Delta)^{2}$ em $H_{0}^{2}(\Omega)$, isto é, com condições de fronteira $u=\frac{\partial u}{\partial v}=0$ sobre $\partial \Omega$, e seja $\left\{w_{n}\right\}_{n \geq 1}$ os correspondentes autofunções. Seja, para todo $n \geq 1$,

$$
H_{n}=\operatorname{span}\left\{w_{1}, \ldots, w_{n}\right\}
$$

Definimos $P_{n}$ e $Q_{n}$ as projeções ortogonais dadas

$$
P_{n}: L^{2}(\Omega) \rightarrow H_{n} \quad \text { e } \quad Q_{n}=I-P_{n}: L^{2}(\Omega) \rightarrow L^{2}(\Omega)-H_{n}
$$

Estas projeções são também ortogonais sobre $H_{0}^{2}(\Omega)$ e satisfazem

$$
\|\phi\|_{2}^{2} \leq \frac{1}{\lambda_{n+1}^{1 / 2}}\|\nabla \phi\|_{2}^{2} \leq \frac{1}{\lambda_{n+1}}\|\Delta \phi\|_{2}^{2}, \quad \forall n \geq 1, \forall \phi \in Q_{n}\left(H_{0}^{2}(\Omega)\right)
$$

Prosseguindo, definimos a correspondente projeção de $E_{0}$ sobre $H_{n} \times H_{n}$,

$$
\mathscr{P}_{n}: E_{0} \rightarrow P_{n}\left(H_{0}^{2}(\Omega)\right) \times P_{n}\left(L^{2}(\Omega)\right) \quad \text { e } \quad \mathscr{Q}_{n}=I-\mathscr{P}_{n}
$$


Sejam $\left(u(t), u_{t}(t)\right)$ e $\left(v(t), v_{t}(t)\right)$ duas soluções de $(2.1)$ com respectivos dados iniciais $U_{0}=$ $\left(u_{0}, u_{1}\right)$ e $V_{0}=\left(v_{0}, v_{1}\right)$, tomados em $X$. Definindo $w=u-v$ então $w$ satisfaz a seguinte equação

$$
\begin{aligned}
w_{t t}+\Delta^{2} w-M\left(\|\nabla u(t)\|_{2}^{2}\right) \Delta w+\beta w_{t}= & \left(M\left(\|\nabla u(t)\|_{2}^{2}\right)-M\left(\|\nabla v(t)\|_{2}^{2}\right)\right) \Delta v \\
& +|u|^{\rho} u-|v|^{\rho} v \text { em } \Omega \times \mathbb{R}^{+}
\end{aligned}
$$

com condições iniciais e de fronteira

$$
w(., 0)=u_{0}-v_{0}, \quad w_{t}(., 0)=u_{1}-v_{1} \quad \text { e } \quad w=\frac{\partial w}{\partial v}=0 \quad \text { sobre } \quad \Gamma \times \mathbb{R}^{+} .
$$

Para deixar mais claro o nosso argumento, separamos parte da demonstração em um lema técnico (Lema 3.4.1), que estabelece a existência de $C=C(X)>0$, independente de $\lambda_{n+1}$, tal que

$$
\left\|S(t) U_{0}-S(t) V_{0}\right\|_{E_{0}} \leq e^{C t}\left\|U_{0}-V_{0}\right\|_{E_{0}} \quad \text { para todo } \quad t \geq 0, \quad U_{0}, V_{0} \in X
$$

$\mathrm{e}$

$$
\left\|\mathscr{Q}_{n} S(t) U_{0}-\mathscr{Q}_{n} S(t) V_{0}\right\|_{E_{0}}^{2} \leq\left(3 e^{-\varepsilon t}+\frac{2 k_{3} e^{C t}}{(C+\varepsilon) \lambda_{n+1}^{1 / 4}}\right)\left\|U_{0}-V_{0}\right\|_{E_{0}}^{2}
$$

Para ver a propriedade da compressão da Definição 1.3.5, suponhamos que (1.7) não se verifica, isto é,

$$
\left\|\mathscr{P}_{n}\left(S(t) U_{0}-S(t) V_{0}\right)\right\|_{E_{0}} \leq\left\|\mathscr{Q}_{n}\left(S(t) U_{0}-S(t) V_{0}\right)\right\|_{E_{0}} .
$$

Logo vemos que

$$
\begin{aligned}
\left\|S(t) U_{0}-S(t) V_{0}\right\|_{E_{0}}^{2} & \leq\left\|\mathscr{P}_{n}\left(S(t) U_{0}-S(t) V_{0}\right)\right\|_{E_{0}}^{2}+\left\|\mathscr{Q}_{n}\left(S(t) U_{0}-S(t) V_{0}\right)\right\|_{E_{0}}^{2} \\
& \leq 2\left\|\mathscr{Q}_{n}\left(S(t) U_{0}-S(t) V_{0}\right)\right\|_{E_{0}}^{2} .
\end{aligned}
$$

Assim, segue de (3.60) e (3.61) que

$$
\left\|S(t) U_{0}-S(t) V_{0}\right\|_{E_{0}}^{2} \leq\left(6 e^{-\varepsilon t}+\frac{4 k_{3} e^{C t}}{(C+\varepsilon) \lambda_{n+1}^{1 / 4}}\right)\left\|U_{0}-V_{0}\right\|_{E_{0}}^{2} .
$$


Como $\lambda_{j} \rightarrow+\infty$ quando $j \rightarrow+\infty$, podemos escolher $t=t_{*}$ e depois $n=n_{0}$, suficientemente grandes, tais que

$$
\left(6 e^{-\varepsilon t}+\frac{4 k_{3} e^{C t}}{(C+\varepsilon) \lambda_{n+1}^{1 / 4}}\right) \leq \frac{1}{8} .
$$

Assim, denotando $S_{*}=S\left(t_{*}\right)$, temos

$$
\left\|S_{*} U-S_{*} V\right\|_{E_{0}}^{2} \leq \frac{1}{8}\|U-V\|_{E_{0}}^{2}, \quad \forall U, V \in X
$$

ou seja, (1.8) é satisfeita. Portanto a propriedade de compressão está verificada.

Por outro lado, de (3.59), vemos que $S\left(t_{*}\right)$ é Lipschitz em $X$, isto é,

$$
\left\|S_{*} U-S_{*} V\right\|_{E_{0}}^{2} \leq L\|U-V\|_{E_{0}}^{2}, \quad \forall U, V \in X,
$$

onde $L=e^{C t_{*}}$

O Teorema 3.4.1 segue então do Teorema 1.3.2

Finalizamos esta seção com a demonstração do lema técnico utilizado no Teorema 3.4.1.

Lema 3.4.1 Nas hipóteses do Teorema 3.4.1, existe uma contante $C=C(X)$, tal que

$$
\left\|S(t) U_{0}-S(t) V_{0}\right\|_{E_{0}} \leq e^{C t}\left\|U_{0}-V_{0}\right\|_{E_{0}}
$$

$e$

$$
\left\|\mathscr{Q}_{n} S(t) U_{0}-\mathscr{Q}_{n} S(t) V_{0}\right\|_{E_{0}}^{2} \leq\left(3 e^{-\varepsilon t}+\frac{2 k_{3} e^{C t}}{(C+\varepsilon) \lambda_{n+1}^{1 / 4}}\right)\left\|U_{0}-V_{0}\right\|_{E_{0}}^{2},
$$

$\forall t \geq 0$ e $U_{0}, V_{0} \in X$

\section{Demonstração:}

Multiplicando a equação de (3.58) por $w_{t}$, integrando sobre $\Omega \times[0, t), t \leq T$ e repetindo argumentos similares aos usados na prova de unicidade de solução deduzimos que

$$
\left\|w_{t}(t)\right\|_{2}^{2}+\|\Delta w(t)\|_{2}^{2} \leq C \int_{0}^{t}\left(\left\|w_{t}(s)\right\|_{2}^{2}+\|\Delta w(s)\|_{2}^{2}\right) d s
$$


Do Lema de Gronwall obtemos

$$
\left\|w_{t}(t)\right\|_{2}^{2}+\|\Delta w(t)\|_{2}^{2} \leq e^{C t}\left\{\left\|w_{t}(0)\right\|_{2}^{2}+\|\Delta w(0)\|_{2}^{2}\right\} \quad \text { para todo } \quad t \geq 0
$$

e portanto,

$$
\left\|S(t) U_{0}-S(t) V_{0}\right\|_{E_{0}} \leq e^{C t}\left\|U_{0}-V_{0}\right\|_{E_{0}} \quad \text { para todo } \quad t \geq 0
$$

Vamos supor por hora que os dados iniciais são tomados em $E_{1}$. Logo as soluções correspondentes possuem a regularidade (2.5). Apliquemos $Q_{n}$ sobre a equação (3.58). Como $Q_{n}$ comuta com $-\Delta, \phi=Q_{n} w$ satisfaz a seguinte equação

$$
\begin{aligned}
\phi_{t t}+\Delta^{2} \phi-M\left(\|\nabla u(t)\|_{2}^{2}\right) \Delta \phi+\beta \phi_{t}= & {\left[M\left(\|\nabla u(t)\|_{2}^{2}\right)-M\left(\|\nabla v(t)\|_{2}^{2}\right)\right] \Delta\left(Q_{n} v\right) } \\
& -Q_{n}\left[|u|^{\rho} u-|v|^{\rho} v\right] .
\end{aligned}
$$

Consideremos o funcional associado a equação (3.64)

$$
E_{\varepsilon}(t)=\left\|\phi_{t}(t)\right\|_{2}^{2}+\|\Delta \phi(t)\|_{2}^{2}+M\left(\|\nabla u(t)\|_{2}^{2}\right)\|\nabla \phi(t)\|_{2}^{2}+\varepsilon \int_{\Omega} \phi_{t} \phi d x
$$

onde $\varepsilon$ é fixado

$$
0<\varepsilon_{0} \leq \varepsilon=\min \{1, \beta\}
$$

e $\lambda_{n+1} \geq 1$. Então, multiplicando (3.64) por $\phi_{t}+\frac{\varepsilon}{2} \phi$ e integrando sobre $\Omega$ obtemos a seguinte igualdade

$$
\begin{aligned}
\frac{1}{2} \frac{d}{d t} E_{\varepsilon}(t)+\left(\beta-\frac{\varepsilon}{2}\right)\left\|\phi_{t}(t)\right\|_{2}^{2}+\frac{\varepsilon}{2}\|\Delta \phi(t)\|_{2}^{2}+\frac{\varepsilon}{2} M\left(\|\nabla u(t)\|_{2}^{2}\right)\|\nabla \phi(t)\|_{2}^{2} \\
+\frac{\beta}{2}\left(\varepsilon \int_{\Omega} \phi \phi_{t} d x\right)+\frac{\varepsilon}{2} \int_{\Omega} Q_{n}\left(|u|^{\rho} u-|v|^{\rho} v\right) Q_{n}(u-v) d x \\
=-\int_{\Omega} Q_{n}\left(|u|^{\rho} u-|v|^{\rho} v\right) \phi_{t} d x+M^{\prime}\left(\|\nabla u(t)\|_{2}^{2}\right) \int_{\Omega} \nabla u_{t} \nabla u d x\|\nabla \phi(t)\|_{2}^{2} \\
+\left(M\left(\|\nabla u(t)\|_{2}^{2}\right)-M\left(\|\nabla v(t)\|_{2}^{2}\right) \int_{\Omega} \Delta\left(Q_{n} v\right)\left(\phi_{t}+\frac{\varepsilon}{2} \phi\right) d x\right.
\end{aligned}
$$

Agora, vamos estimar os termos do lado direito de (3.65). A regularidade (2.5) será fundamental aqui. Novamente, usando a desigualdade de Hölder com $\frac{\rho}{2(\rho+1)}+\frac{1}{2(\rho+1)}+\frac{1}{2}=1$, resultados de 
imersão $H_{0}^{2}(\Omega) \hookrightarrow H_{0}^{1}(\Omega) \hookrightarrow L^{2(\rho+1)}(\Omega)$ e a desigualdade de Young, resulta que

$$
\begin{aligned}
& -\int_{\Omega} Q_{n}\left(|u|^{\rho} u-|v|^{\rho} v\right) \phi_{t} d x \\
& \quad \leq k_{0}(\rho)\left(\|u(t)\|_{2(\rho+1)}^{\rho}+\|v(t)\|_{2(\rho+1)}^{\rho}\right)\|w(t)\|_{2(\rho+1)}\left\|\phi_{t}(t)\right\|_{2} \\
& \quad \leq \frac{k_{1}(\rho)}{\lambda_{n+1}^{1 / 4}}\|\Delta w(t)\|_{2}\left\|\nabla \phi_{t}(t)\right\|_{2} .
\end{aligned}
$$

Como estamos interessados em estimativas para $t$ grande, segue que

$$
\left\|\nabla \phi_{t}(t)\right\|_{2} \leq\left\|\nabla u_{t}(t)\right\|_{2}+\left\|\nabla v_{t}(t)\right\|_{2} \leq k_{2}\left(r_{1}\right) .
$$

Retornando a (3.66), obtemos

$$
-\int_{\Omega} Q_{n}\left(|u|^{\rho} u-|v|^{\rho} v\right) \phi_{t} d x \leq \frac{k_{3}\left(\rho, r_{1}\right)}{\lambda_{n+1}^{1 / 4}}\|\Delta w(t)\|_{2}^{2} .
$$

Os outros termos do lado direito de (3.65) são estimados a seguir. Em ordem, pela continuidade de $M^{\prime}$ obtemos

$$
\begin{aligned}
& M^{\prime}\left(\|\nabla u(t)\|_{2}^{2}\right) \int_{\Omega} \nabla u_{t} \nabla u d x\|\nabla \phi(t)\|_{2}^{2} \\
& \quad \leq \max _{0 \leq s \leq r_{0} \lambda_{1}^{-1 / 2}} \mid M^{\prime}(s)\left\|\nabla u_{t}(t)\right\|_{2}\|\nabla u(t)\|_{2}\|\nabla \phi(t)\|_{2}^{2} \leq \frac{k_{4}\left(r_{0}, r_{1}\right)}{\lambda_{n+1}^{1 / 4}}\|\Delta w(t)\|_{2}^{2} .
\end{aligned}
$$

Prosseguindo, pelo Teorema do Valor Médio, temos que

$$
\begin{aligned}
& \left(M\left(\|\nabla u\|_{2}^{2}\right)-M\left(\|\nabla v\|_{2}^{2}\right) \int_{\Omega} \Delta\left(Q_{n} v\right)\left(\frac{\varepsilon}{2} \phi\right) d x\right. \\
& \leq \frac{\varepsilon}{2} \max _{0 \leq s \leq r_{0} \lambda_{1}^{-1 / 2}}\left|M^{\prime}(s)\right|\left[\|\nabla u\|_{2}+\|\nabla v\|_{2}\right]\|\nabla w(t)\|_{2}\|\Delta v(t)\|_{2}\|\phi(t)\|_{2} \\
& \leq \frac{k_{5}\left(r_{0}\right)}{\lambda_{n+1}^{1 / 2}}\|\Delta w(t)\|_{2}\|\Delta \phi(t)\|_{2} \leq \frac{k_{5}\left(r_{0}\right)}{\lambda_{n+1}^{1 / 2}}\|\Delta w(t)\|_{2}^{2}
\end{aligned}
$$


$\mathrm{e}$

$$
\begin{aligned}
& \left(M\left(\|\nabla u\|_{2}^{2}\right)-M\left(\|\nabla v\|_{2}^{2}\right) \int_{\Omega} \Delta\left(Q_{n} v\right) \phi_{t} d x\right. \\
& \quad \leq \frac{\varepsilon}{2} \max _{0 \leq s \leq r_{0} \lambda_{1}^{-1 / 2}}\left|M^{\prime}(s)\right|\left[\|\nabla u\|_{2}+\|\nabla v\|_{2}\right]\|\nabla w(t)\|_{2}\left\|\Delta Q_{n} v(t)\right\|_{2}\left\|\phi_{t}(t)\right\|_{2} \\
& \quad \leq \frac{\varepsilon}{2} \max _{0 \leq s \leq r_{0} \lambda_{1}^{-1 / 2}}\left|M^{\prime}(s)\right|\left[\|\nabla u\|_{2}+\|\nabla v\|_{2}\right]\|\nabla w(t)\|_{2} \frac{1}{\lambda_{n+1}}\left\|\Delta^{2} Q_{n} v(t)\right\|_{2}\left\|\phi_{t}(t)\right\|_{2} \\
& \quad \leq \frac{k_{6}\left(r_{0}, r_{1}\right)}{\lambda_{n+1}}\left(\left\|w_{t}(t)\right\|_{2}^{2}+\|\Delta w(t)\|_{2}^{2}\right) .
\end{aligned}
$$

Portanto, usando as estimativas (3.67)-(3.70) e $\varepsilon$ préviamente fixado, retornando a (3.65), obtemos

$$
\frac{d}{d t} E_{\varepsilon}(t)+\varepsilon E_{\varepsilon}(t) \leq \frac{k_{7}}{\lambda_{n+1}^{1 / 4}}\left(\left\|w_{t}(t)\right\|_{2}^{2}+\|\Delta w(t)\|_{2}^{2}\right) .
$$

Usando (3.63), resulta que

$$
\frac{d}{d t} E_{\varepsilon}(t)+\varepsilon E_{\varepsilon}(t) \leq \frac{k_{7} e^{C t}}{\lambda_{n+1}^{1 / 4}}\left\|U_{0}-V_{0}\right\|_{E_{0}}^{2} .
$$

Multiplicando por $e^{\varepsilon t}$, obtemos

$$
\frac{d}{d t}\left(E_{\varepsilon}(t) e^{\varepsilon t}\right) \leq \frac{k_{7} e^{(C+\varepsilon) t}}{\lambda_{n+1}^{1 / 4}}\left\|U_{0}-V_{0}\right\|_{E_{0}}^{2}
$$

Integrando de 0 até $t$ resulta que

$$
E_{\varepsilon}(t) \leq e^{-\varepsilon t} E_{\varepsilon}(0)+\frac{k_{7} e^{C t}}{(C+\varepsilon) \lambda_{n+1}^{1 / 4}}\left\|U_{0}-V_{0}\right\|_{E_{0}}^{2} .
$$

Por outro lado, como

$$
\left|\varepsilon \int_{\Omega} \phi \phi_{t} d x\right| \leq \frac{\varepsilon^{2}}{2 \lambda_{n+1}^{1 / 4}}\|\Delta \phi(t)\|_{2}^{2}+\frac{1}{2}\left\|\phi_{t}(t)\right\|_{2}^{2} .
$$


Usando que $\lambda_{n+1} \geq 1$, encontramos

$$
-\frac{1}{2}\left\{\|\Delta \phi(t)\|_{2}^{2}+\left\|\phi_{t}(t)\right\|_{2}^{2}\right\} \leq \varepsilon \int_{\Omega} \phi \phi_{t} d x \leq \frac{1}{2}\left\{\|\Delta \phi(t)\|_{2}^{2}+\left\|\phi_{t}(t)\right\|_{2}^{2}\right\} .
$$

Isto implica que

$$
\frac{1}{2}\left\{\|\Delta \phi(t)\|_{2}^{2}+\left\|\phi_{t}(t)\right\|_{2}^{2}\right\} \leq E_{\varepsilon}(t) \leq \frac{3}{2}\left\{\|\Delta \phi(t)\|_{2}^{2}+\left\|\phi_{t}(t)\right\|_{2}^{2}\right\}
$$

Portanto, segue de (3.71) e (3.72) que

$$
\left\|\mathscr{Q}_{n} S\left(U_{0}\right)-\mathscr{Q}_{n} S\left(V_{0}\right)\right\|_{E_{0}}^{2} \leq\left(3 e^{-\varepsilon t}+\frac{2 k_{3} e^{C t}}{(C+\varepsilon) \lambda_{n+1}^{1 / 4}}\right)\left\|U_{0}-V_{0}\right\|_{E_{0}}^{2}
$$

para $U_{0}, V_{0} \in B_{1}$.

Por densidade, a desigualdade vale para todos $U_{0}, V_{0} \in X$ 


\section{Capítulo}

\section{Atrator Global para o Problema com}

\section{Dissipação Não Linear}

Neste capítulo consideramos a equação de vigas extensíveis no caso em que a dissipação é não linear, do tipo $g\left(u_{t}\right) \approx\left|u_{t}\right|^{r} u_{t}$. Neste contexto, discutiremos sobre a existência de solução global e provamos que o semigrupo $S(t)$ associado ao problema possui um atrator global em $E_{0}=H_{0}^{2}(\Omega) \times L^{2}(\Omega)$.

O problema estudado se escreve na forma

$$
\left\{\begin{array}{l}
u_{t t}+\Delta^{2} u-M\left(\|\nabla u(t)\|_{2}^{2}\right) \Delta u+g(u)+f\left(u_{t}\right)=h \quad \text { em } \quad \Omega \times \mathbb{R}^{+} \\
u=\frac{\partial u}{\partial v}=0 \quad \text { sobre } \quad \Gamma \times \mathbb{R}^{+}, \\
u(\cdot, 0)=u_{0} \quad \text { e } \quad u_{t}(\cdot, 0)=u_{1} \quad \text { em } \quad \Omega
\end{array}\right.
$$

onde $\Omega$ é um domínio limitado do $\mathbb{R}^{N}$ com fronteira suave $\Gamma=\partial \Omega$. Como antes, $M$ é uma função satisfazendo (2.3) e (2.4).

No que segue para simplificar a compreensão das estimativas, vamos assumir que

$$
g(u) \approx|u|^{\rho} u \quad \text { e } \quad f\left(u_{t}\right) \approx\left|u_{t}\right|^{r} u_{t}
$$


onde $\rho$ e $r$ são constantes positivas tais que

$$
0<r, \rho \leq \frac{2}{N-2} \text { se } N \geq 3 \text { e } \rho, r>0 \text { se } N=1,2
$$

Observação 4.0.1 Os resultados obtidos para $g(u) \approx|u|^{\rho}$ u e $f\left(u_{t}\right) \approx\left|u_{t}\right|^{r} u_{t}$ podem ser também obtidos assumindo g e f mais gerais, com as seguintes hipóteses:

(i) $f$ e g são de classe $C^{1}$, com $f(0)=g(0)=0,\left|g^{\prime}(u)\right| \leq k_{0}\left(1+|u|^{\rho}\right)$ e $f^{\prime}(v) \geq 0$, para todo $u, v \in \mathbb{R}$, onde $\rho$ satisfaz (4.2).

(ii) Existem constantes $k_{1}, L_{0}, L_{1}>0$ tais que

$$
|g(u)-g(v)| \leq k_{1}\left(1+|u|^{\rho}+|v|^{\rho}\right)|u-v|, \quad \forall u, v \in \mathbb{R}
$$

$e$

$$
-L_{0} \leq \widehat{g}(u) \leq \frac{1}{2} g(u) u+L_{1}, \forall u \in \mathbb{R}
$$

onde $\widehat{g}(z)=\int_{0}^{z} g(s) d s$.

(iii) Existem também constantes $k_{2}, k_{3}>0$ tais que

$$
|f(u)-f(v)| \leq k_{2}\left(1+|u|^{r}+|v|^{r}\right)|u-v|, \quad \forall u, v \in \mathbb{R}
$$

$e$

$$
(f(u)-f(v))(u-v) \geq k_{3}|u-v|^{r+2}, \forall u, v \in \mathbb{R},
$$

onde $r>0$ satisfaz, (4.2).

Os detalhes pode ser encontrados em [33].

O resultado principal do capítulo é o Teorema 4.2.1. 


\subsection{Existência de Solução Global}

Nossa análise será realizada no espaço de Sobolev

$$
E_{0}=H_{0}^{2}(\Omega) \times L^{2}(\Omega)
$$

equipado com a norma

$$
\|(u, v)\|_{E_{0}}^{2}=\|\Delta u\|_{2}^{2}+\|v\|_{2}^{2}
$$

Note que a hipótese (4.2) implica que $H_{0}^{2}(\Omega) \hookrightarrow H_{0}^{1}(\Omega) \hookrightarrow L^{2(q+1)}(\Omega)$, onde $q=\rho$ ou $q=r$. A energia do sistema (4.1) é definida por

$$
E(t)=\frac{1}{2}\left\|u_{t}(t)\right\|_{2}^{2}+\frac{1}{2}\|\Delta u(t)\|_{2}^{2}+\frac{1}{2} \widehat{M}\left(\|\nabla u(t)\|_{2}^{2}\right)+\frac{1}{\rho+2}\|u(t)\|_{\rho+2}^{\rho+2}-\int_{\Omega} h u(t) d x .
$$

Quando $h=0$, o problema (4.1) foi estudado por Cavalcanti et al. [14], onde a existência global e o decaimento polinomial da energia foram obtidos. Para deixar o texto mais auto-suficiente, faremos um breve comentário sobre estes resultados.

Teorema 4.1.1 (Cavalcanti, Domingos Cavalcanti \& Soriano) Suponhamos que as condições (2.3)-(2.4) e (4.2) são válidas e que $h=0$. Então, se os dados iniciais $\left(u_{0}, u_{1}\right)$ são tomados em

$$
E_{1}=\left(H^{4}(\Omega) \cap H_{0}^{2}(\Omega)\right) \times H_{0}^{2}(\Omega),
$$

o problema (4.1) possui uma única solução regular $u=u(x, t)$ satisfazendo

$$
u \in L^{\infty}\left(\mathbb{R}^{+} ; H^{4}(\Omega) \cap H_{0}^{2}(\Omega)\right), \quad u_{t} \in L^{\infty}\left(\mathbb{R}^{+} ; H_{0}^{2}(\Omega)\right), \quad u_{t t} \in L^{\infty}\left(\mathbb{R}^{+} ; L^{2}(\Omega)\right) .
$$

Por outro lado, se os dados iniciais $\left(u_{0}, u_{1}\right)$ são tomados em $E_{0}=H_{0}^{2}(\Omega) \times L^{2}(\Omega)$, o problema (4.1) possui uma única solução fraca u satisfazendo

$$
u \in C^{0}\left([0, \infty) ; H_{0}^{2}(\Omega)\right) \cap C^{1}\left([0, \infty) ; L^{2}(\Omega)\right) .
$$


Em ambos os casos, a energia $E(t)$ possui a taxa de decaimento

$$
E(t) \leq\left(\theta t+E(0)^{-\frac{r}{2}}\right)^{-\frac{2}{r}}, \quad \forall t \geq 0
$$

onde $\theta>0$ é uma constante positiva.

Para nosso propósito, vamos admitir que o resultado de existência de solução global do o Teorema 4.1.1 é válido quando $h \in L^{2}(\Omega)$ não é necessariamente zero. Isto pode ser visto relembrando os principais argumentos da prova do Teorema 4.1.1, que é dada em várias etapas.

1 - Considera-se um problema simplificado, substituindo-se a equação principal em (4.1) por

$$
u_{t t}+\Delta^{2} u-\mu(t) \Delta u+f(u)+g\left(u_{t}\right)=0
$$

com dados em $E_{1}$. Então a existência de uma única solução global regular é obtida usando o método de Faedo-Galerkin.

2 - Define-se um operador de ponto fixo

$$
\mathscr{S}: v \mapsto z
$$

em um subespaço conveniente de $L^{\infty}\left(0, T_{0} ; H_{0}^{2}(\Omega)\right)$, onde $z$ é a única solução do problema simplificado acima com $\mu(t)=M\left(\|\nabla v(t)\|_{2}^{2}\right)$. Então a existência de uma única solução local de (4.1) é provada usando estimativas do problema simplificado e o teorema de ponto fixo para contrações.

3 - Seja $u$ a única solução local obtida na etapa anterior. Então tomando $\mu(t)=M\left(\|\nabla u(t)\|_{2}^{2}\right)$, e tendo em vista a unicidade de ambos os problemas original e simplificado, a existência de uma global solução de (4.1) é finalmente obtida. Aqui, o principal argumento baseia-se no lema de Zorn.

4 - A existência de solução fraca em $E_{0}$ é obtida usando argumentos de densidade. Tomando os dados iniciais $\left(u_{0}, u_{1}\right) \in E_{0}$, então existe uma sequência $\left(u_{0}^{v}, u_{1}^{v}\right) \in E_{1}$ tal que

$$
u_{0}^{v} \rightarrow u_{0} \text { em } H_{0}^{2}(\Omega) \quad \text { e } \quad u_{1}^{v} \rightarrow u_{1} \text { em } L^{2}(\Omega)
$$

Então estimativas uniformes sobre as correspondentes soluções regulares $u^{v}$ mostram a existência de uma subsequência $u^{v_{k}}$ que converge para a única solução fraca $u$ do problema (4.1), 
satisfazendo

$$
u_{t t}+\Delta^{2} u-M\left(\int_{\Omega}|\nabla u|^{2} d x\right) \Delta u+|u|^{\rho} u+\left|u_{t}\right|^{r} u_{t}=0 \quad \text { em } \quad L_{l o c}^{2}\left(\mathbb{R}^{+} ; H^{-2}(\Omega)+L^{\frac{r+2}{r+1}}(\Omega)\right) .
$$

Observação 4.1.1 No Teorema 4.1.1, a taxa de decaimento (4.6) para as soluções regulares é obtida através de métodos de multiplicadores, onde a diferenciação de $E(t)$ é necessária. Quando consideramos soluções fracas, $E^{\prime}(t)=-\left\|u_{t}(t)\right\|_{r+2}^{r+2}$ não é válida em geral, de modo que argumentos de densidade são necessários. Notamos que, das estimativas ou de (4.6), as soluções (regulares ou fracas) satisfazem

$$
\left\|u_{t}(t)\right\|_{2}^{2}+\|\Delta u(t)\|_{2}^{2}+\|u(t)\|_{\rho+2}^{\rho+2} \leq C, \quad \forall t \geq 0
$$

onde $C>0$ é uma constante dependente apenas do dados iniciais.

Observação 4.1.2 Em vista do Teorema 4.1.1, com $h \in L^{2}(\Omega)$, podemos definir sobre $E_{0}$ um operador

$$
S(t):\left(u_{0}, u_{1}\right) \mapsto\left(u(t), u_{t}(t)\right), \quad t \geq 0,
$$

onde $u(t)$ é a única solução de (4.1). Segue que $\{S(t)\}_{t \geq 0}$ é um $C_{0}$-semigrupo não linear definido sobre o espaço de fase $E_{0}$.

\subsection{Existência de Atrator Global}

Teorema 4.2.1 Suponhamos que as hipóteses (2.3)-(2.4) e (4.2) são satisfeitas, com $h \in L^{2}(\Omega)$. Então o semigrupo $\{S(t)\}_{t \geq 0}$ associado ao problema (4.1) possui um atrator global em $E_{0}$.

\section{Demonstração:}

Primeiramente observamos que semigrupo $\{S(t)\}_{t \geq 0}$ possui um conjunto absorvente $B_{0} \subset$ $E_{0}$, que é provado no Lemma 4.2.1 abaixo. Em seguida, no Lemma 4.2.3 é provado que $\{S(t)\}_{t \geq 0}$ é assintoticamente suave em $E_{0}$. Assim pelo Lema 1.3.2, $\{S(t)\}_{t \geq 0}$ é assintoticamente compacto. Portanto a existência de um atrator global para $\{S(t)\}_{t \geq 0}$ é garantida pelo Teorema 1.3.1.

O restante do capítulo é dedicado aos Lemas 4.2.1 e 4.2.3 . 


\subsubsection{Conjunto absorvente em $E_{0}$}

A existência de um conjunto absorvente é assegurada pelo seguinte Lema.

Lema 4.2.1 Sob as hipóteses do Teorema 4.2.1, o semigrupo $\{S(t)\}_{t \geq 0}$ associado ao problema (4.1) possui um conjunto absorvente $B_{0} \subset E_{0}$.

\section{Demonstração:}

Seja $B \subset E_{0}$ um subconjunto limitado arbitrário e consideremos as soluções do problema (4.1) dadas por $\left(u(t), u_{t}(t)\right)=S(t)\left(u_{0}, u_{1}\right) \operatorname{com}\left(u_{0}, u_{1}\right) \in B$.

Nossa análise é baseada na energia modificada

$$
\begin{aligned}
\widetilde{E}(t)= & \frac{1}{2}\left(\|\Delta u(t)\|_{2}^{2}+\widehat{M}\left(\|\nabla u(t)\|_{2}^{2}\right)+\frac{2}{\rho+2}\|u(t)\|_{\rho+2}^{\rho+2}\right) \\
& +\frac{1}{2}\left\|u_{t}(t)\right\|_{2}^{2}-\int_{\Omega} h u(t) d x+\frac{1}{\lambda_{1}}\|h\|_{2}^{2},
\end{aligned}
$$

onde $\lambda_{1}>0$ é o primeiro autovalor do operador bi-harmônico $\Delta^{2}$ em $H_{0}^{2}(\Omega)$. Assim, $\widetilde{E}(t)=$ $E(t)+\frac{1}{\lambda_{1}}\|h\|_{2}^{2}$ e $\widetilde{E}(t)$ domina $\left\|\left(u(t), u_{t}(t)\right)\right\|_{E_{0}}^{2}$. De fato, como $\lambda_{1}$ satisfaz

$$
\|u\|_{2}^{2} \leq \lambda_{1}^{-1}\|\Delta u\|_{2}^{2}, \quad \forall u \in H_{0}^{2}(\Omega)
$$

temos que

$$
\int_{\Omega} h(x) u(x, t) d x \leq \frac{1}{\lambda_{1}}\|h\|_{2}^{2}+\frac{1}{4}\|\Delta u(t)\|_{2}^{2}
$$

e portanto

$$
\widetilde{E}(t) \geq \frac{1}{4}\|\Delta u(t)\|_{2}^{2}+\frac{1}{2}\left\|u_{t}(t)\right\|_{2}^{2} .
$$

Para lidar com esta energia modificada usamos argumentos de densidade como mencionado na Observação 4.1.1. Isto é, podemos assumir formalmente que $u$ é uma solução regular do problema.

Multiplicando a primeira equação de (4.1) por $u_{t}$ e integrando sobre $\Omega$, obtemos

$$
\frac{d}{d t} E(t)=-\left\|u_{t}(t)\right\|_{r+2}^{r+2}
$$


e então, integrando de $t$ até $t+1$, encontramos

$$
E(t)-E(t+1)=\int_{t}^{t+1} \int_{\Omega}\left|u_{t}(x, s)\right|^{r+2} d x d s \geq 0 .
$$

Assim, podemos definir um funcional auxiliar $F(t)$ por

$$
F(t)^{2}=\widetilde{E}(t)-\widetilde{E}(t+1)=E(t)-E(t+1) .
$$

No que segue, iremos comparar $\widetilde{E}(t)^{1+\frac{r}{2}} \operatorname{com} F(t)^{2}$, visando aplicar o Lema de Nakao (Lema 1.3.1) $\operatorname{com} \phi(t)=\widetilde{E}(t)$ e $\gamma=\frac{r}{2}$.

Agora, multiplicando a primeira equação de (4.1) por $u$ e integrando sobre $\Omega$, podemos ver que

$$
\begin{aligned}
& \|\Delta u(t)\|_{2}^{2}+M\left(\|\nabla u(t)\|_{2}^{2}\right)\|\nabla u(t)\|_{2}^{2}+\|u(t)\|_{\rho+2}^{\rho+2} \\
& \quad=\left\|u_{t}(t)\right\|_{2}^{2}-\frac{d}{d t} \int_{\Omega} u_{t}(t) u(t) d x+\int_{\Omega}\left|u_{t}(t)\right|^{r} u_{t}(t) u(t) d x+\int_{\Omega} h u(t) d x .
\end{aligned}
$$

Então, combinando (4.13) com (4.9) vemos

$$
\begin{aligned}
\widetilde{E}(t) \leq & \left\|u_{t}(t)\right\|_{2}^{2}-\frac{1}{2} \frac{d}{d t} \int_{\Omega} u_{t}(t) u(t) d x-\frac{1}{2} \int_{\Omega} h u(t) d x \\
& +\frac{1}{2} \int_{\Omega}\left|u_{t}(t)\right|^{r} u_{t}(t) u(t) d x+\frac{1}{\lambda_{1}}\|h\|_{2}^{2},
\end{aligned}
$$

e portanto

$$
\begin{aligned}
\int_{t_{1}}^{t_{2}} \widetilde{E}(s) d s \leq & \int_{t_{1}}^{t_{2}}\left\|u_{t}(s)\right\|_{2}^{2} d s-\frac{1}{2}\left(\int_{\Omega} u_{t}\left(t_{2}\right) u\left(t_{2}\right) d x-\int_{\Omega} u_{t}\left(t_{1}\right) u\left(t_{1}\right) d x\right) \\
& -\frac{1}{2} \int_{t_{1}}^{t_{2}} \int_{\Omega} h u(s) d x d s+\frac{1}{2} \int_{t_{1}}^{t_{2}} \int_{\Omega}\left|u_{t}(s)\right|^{r} u_{t} u(s) d x d s \\
& +\frac{1}{\lambda_{1}} \int_{t_{1}}^{t_{2}}\|h\|_{2}^{2} d s .
\end{aligned}
$$

Agora, vamos estimar o lado direito de (4.15) em termos de $F(t)$. Usaremos $C_{0}$ como uma constante positiva genérica que não depende dos dados iniciais. 
Em vista de (4.12) temos, como $\frac{r}{r+2}+\frac{2}{r+2}=1$,

$$
\int_{t}^{t+1}\left\|u_{t}(s)\right\|_{2}^{2} d s \leq|\Omega|^{\frac{r}{r+2}}\left(\int_{t}^{t+1} \int_{\Omega}\left|u_{t}(x, s)\right|^{r+2} d x d s\right)^{\frac{2}{r+2}}=|\Omega|^{\frac{r}{r+2}} F(t)^{\frac{4}{r+2}}
$$

Então, pelo Teorema do Valor Médio para integrais , existem $t_{1} \in\left[t, t+\frac{1}{4}\right]$ e $t_{2} \in\left[t+\frac{3}{4}, t+1\right]$ tais que

$$
\left\|u_{t}\left(t_{i}\right)\right\|_{2}^{2} \leq 4|\Omega|^{\frac{r}{r+2}} F(t)^{\frac{4}{r+2}}, \quad i=1,2 .
$$

Combinando (4.10) com (4.17) e então notando que $\|\Delta u(s)\|_{2} \leq 2 \widetilde{E}(s)^{\frac{1}{2}}$, temos

$$
\begin{aligned}
\left|\int_{\Omega} u_{t}\left(t_{2}\right) u\left(t_{2}\right) d x-\int_{\Omega} u_{t}\left(t_{1}\right) u\left(t_{1}\right) d x\right| & \leq \frac{4}{\lambda_{1}^{1 / 2}}|\Omega|^{\frac{r}{2(r+2)}} F(t)^{\frac{2}{r+2}} \sup _{t \leq s \leq t+1}\|\Delta u(s)\|_{2} \\
& \leq C_{0} F(t)^{\frac{4}{r+2}}+\frac{1}{8} \sup _{t \leq s \leq t+1} \widetilde{E}(s) .
\end{aligned}
$$

Analogamente, de $\frac{r+1}{r+2}+\frac{1}{r+2}=1$ e (4.16), obtemos

$$
\begin{aligned}
\int_{t_{1}}^{t_{2}} \int_{\Omega}\left|u_{t}(s)\right|^{r+1}|u(s)| d x d s & \leq\left(\int_{t}^{t+1} \int_{\Omega}\left|u_{t}(s)\right|^{r+2} d x d s\right)^{\frac{r+1}{r+2}}\left(\int_{t}^{t+1} \int_{\Omega}|u(s)|^{r+2} d x d s\right)^{\frac{1}{r+2}} \\
& \leq F(t)^{\frac{2(r+1)}{r+2}} \sup _{t \leq s \leq t+1}\|u(s)\|_{r+2} \\
& \leq F(t)^{\frac{2(r+1)}{r+2}} \mu \sup _{t \leq s \leq t+1}\|\Delta u(s)\|_{2},
\end{aligned}
$$

onde $\mu$ é a constante de imersão de $H_{0}^{2}(\Omega)$ em $L^{r+2}(\Omega)$. Assim, como antes,

$$
\int_{t_{1}}^{t_{2}} \int_{\Omega}\left|u_{t}(s)\right|^{r+1}|u(s)| d x d s \leq C_{0} F(t)^{\frac{4(r+1)}{r+2}}+\frac{1}{8} \sup _{t \leq s \leq t+1} \widetilde{E}(s) .
$$

Finalmente, observamos que

$$
\begin{aligned}
\int_{t_{1}}^{t_{2}} \int_{\Omega} h u(s) d x d s & \leq \frac{1}{\lambda_{1}^{1 / 2}}\|h\|_{2} \sup _{t \leq s \leq t+1}\|\Delta u(s)\|_{2} \\
& \leq C_{0}\|h\|_{2}^{2}+\frac{1}{8} \sup _{t \leq s \leq t+1} \widetilde{E}(s) .
\end{aligned}
$$


Portanto, inserindo (4.16), (4.18), (4.19) e (4.20) em (4.15), obtemos

$$
\int_{t_{1}}^{t_{2}} \widetilde{E}(s) d s \leq C_{0}\left(F(t)^{\frac{4}{r+2}}+F(t)^{\frac{4(r+1)}{r+2}}\right)+C_{0}\|h\|_{2}^{2}+\frac{3}{8} \sup _{t \leq s \leq t+1} \widetilde{E}(s) .
$$

Usando novamente o Teorema do Valor Médio para integrais, existe $\tau \in\left[t_{1}, t_{2}\right]$ tal que

$$
\int_{t_{1}}^{t_{2}} \widetilde{E}(s) d s=\widetilde{E}(\tau)\left(t_{2}-t_{1}\right) \geq \frac{1}{2} \widetilde{E}(t+1)
$$

pois $\widetilde{E}(t)$ é decrescente como $E(t)$. Então, de $\widetilde{E}(t+1)=\widetilde{E}(t)-F(t)^{2}$, concluimos que

$$
\widetilde{E}(t) \leq F(t)^{2}+2 \int_{t_{1}}^{t_{2}} \widetilde{E}(s) d s
$$

Combinado (4.21) e (4.22) vemos

$$
\widetilde{E}(t) \leq F(t)^{2}+C_{0}\left(F(t)^{\frac{4}{r+2}}+F(t)^{\frac{4(r+1)}{r+2}}\right)+C_{0}\|h\|_{2}^{2}+\frac{3}{4} \widetilde{E}(t)
$$

e como $\frac{4}{r+2}<2<\frac{4(r+1)}{r+2}$

$$
\widetilde{E}(t) \leq C_{0} F(t)^{\frac{4}{r+2}}\left(1+F(t)^{\frac{2 r}{r+2}}+F(t)^{\frac{4 r}{r+2}}\right)+C_{0}\|h\|_{2}^{2}
$$

Além disso, da definição de $F(t)$ e estimativa (4.8), inferimos que $\left(1+F(t)^{\frac{24}{r+2}}+F(t)^{\frac{4 r}{r+2}}\right)$ é limitado por uma constante $C_{0}^{\prime}>0$, que é dependente de $B$. Assim, podemos reescrever (4.24) como

$$
\widetilde{E}(t) \leq C_{0} C_{0}^{\prime} F(t)^{\frac{4}{r+2}}+C_{0}\|h\|_{2}^{2}
$$

que implica que

$$
\widetilde{E}(t)^{1+\frac{r}{2}} \leq C_{B}(\widetilde{E}(t)-\widetilde{E}(t+1))+C_{0}\|h\|_{2}^{r+2},
$$

onde $C_{B}>0$ é uma constante dependente de $B$, mas $C_{0}>0$ não. Portanto podemos aplicar o Lema Nakao, com $\phi=\widetilde{E}$ and $\gamma=\frac{r}{2}$, para concluir que

$$
\widetilde{E}(t) \leq\left(C_{B}^{-1} \frac{r}{2}(t-1)^{+}+\widetilde{E}(0)^{-\frac{r}{2}}\right)^{-\frac{2}{r}}+C_{0}^{\frac{2}{r+2}}\|h\|_{2}^{2} .
$$


Como o primeiro termo do lado direito de (4.27) vai pra zero com $t \rightarrow \infty$, existe $t_{B}>0$, dependente de $B$, tal que

$$
\widetilde{E}(t) \leq 2 C_{0}^{\frac{2}{r+2}}\|h\|_{2}^{2}, \quad \forall t>t_{B}
$$

Portanto, usando (4.11) concluimos que

$$
\|\Delta u(t)\|_{2}^{2}+\|v(t)\|_{2}^{2} \leq 4 \widetilde{E}(t) \leq 8 C_{0}^{\frac{2}{r+2}}\|h\|_{2}^{2}, \quad \forall t>t_{B},
$$

ou seja,

$$
B_{0}=\left\{(u, v) \in E_{0} \mid\|\Delta u\|_{2}^{2}+\|v\|_{2}^{2} \leq r_{0}\right\}
$$

onde $r_{0}=8 C_{0}^{\frac{2}{r+2}}\|h\|_{2}^{2}$, é um conjunto absorvente para $S(t)$.

Observação 4.2.1 A estimativa (4.27) mostra que, quando $h=0$, a energia $E(t)$ possui a mesma taxa de decaimento polinomial obtida por Cavalcanti et al. no Teorema 4.1.1.

\subsubsection{Compacidade assintótica em $E_{0}$}

Para provar a propriedade de compacidade assintótica requerida pelo do Teorema 1.3.1, aplicamos o Lema 1.3.2.

Dados dois pares de dados iniciais $\left(u_{0}, u_{1}\right),\left(v_{0}, v_{1}\right) \in E_{0}$, sejam $u, v$ soluções correspondentes do problema (4.1). Então do Teorema 4.1.1, w $=u-v$ é solução fraca do seguinte problema

$$
\left\{\begin{array}{l}
w_{t t}+\Delta^{2} w+\left(\left|u_{t}\right|^{r} u_{t}-\left|v_{t}\right|^{r} v_{t}\right) \\
\quad=M\left(\|\nabla u\|_{2}^{2}\right) \Delta u-M\left(\|\nabla v\|_{2}^{2}\right) \Delta v+\left(|u|^{\rho} u-|v|^{\rho} v\right) \quad \text { em } \quad \Omega \times \mathbb{R}^{+} \\
w=\frac{\partial u}{\partial v}=0 \quad \text { sobre } \quad \Gamma \times \mathbb{R}^{+} \\
w(\cdot, 0)=u_{0}-v_{0}, \quad w_{t}(\cdot, 0)=u_{1}-v_{1} \quad \text { em } \quad \Omega
\end{array}\right.
$$

Definimos o funcional

$$
E_{w}(t)=\left\|w_{t}(t)\right\|_{2}^{2}+\|\Delta w(t)\|_{2}^{2}+M\left(\|\nabla u(t)\|_{2}^{2}\right)\|\nabla w(t)\|_{2}^{2} .
$$

Como $M \geq 0$, segue que $E_{w}$ domina a norma de $E_{0}$. 
Usaremos o Lema de Nakao novamente para provar $E_{w}(t)$ satisfaz uma desigualdade preliminar.

Lema 4.2.2 Seja $T>0$ e suponha que os dados iniciais estão num conjunto limitado $B \subset E_{0}$. Então existem constantes positivas $C_{B}$ e $C_{T}, \operatorname{com} C_{B}$ não dependente de $T$, tais que

$$
E_{w}(t) \leq C_{B}(t-1)^{-\frac{2}{r}}+C_{T}\left(\sup _{0 \leq \sigma \leq T} \int_{\sigma}^{\sigma+1}\|\nabla w(s)\|_{2} d s\right)^{\frac{2}{r+2}}, \quad 0 \leq t \leq T .
$$

\section{Demonstração:}

A prova é semelhante a do Lema 4.2.1. Para estimar $E_{w}(t)$, por argumentos de densidade, assumimos que $w=u-v$ é uma solução regular de (4.29).

Multiplicando a equação de (4.29) por $w_{t}$ e integrando sobre $\Omega$, obtemos

$$
\begin{aligned}
\frac{1}{2} \frac{d}{d t} E_{w}(t)+ & \int_{\Omega}\left(g\left(u_{t}(t)\right)-g\left(v_{t}(t)\right)\right) w_{t}(t) d x \\
= & M^{\prime}\left(\|\nabla u(t)\|_{2}^{2}\right) \int_{\Omega} u_{t}(t) \Delta u(t) d x\|\nabla w(t)\|_{2}^{2} \\
& +\left(M\left(\|\nabla u(t)\|_{2}^{2}\right)-M\left(\|\nabla v(t)\|_{2}^{2}\right)\right) \int_{\Omega} \Delta v(t) w_{t}(t) d x \\
& -\int_{\Omega}(f(u(t))-f(v(t))) w_{t}(t) d x
\end{aligned}
$$

Para estimar o lado direito de (4.31), usaremos $C_{1}>0$ como uma constante genérica dependente de $B$ mas não de $t$.

A continuidade de $M^{\prime}$ e estimativa (4.8) mostram que

$$
M^{\prime}\left(\|\nabla u(t)\|_{2}^{2}\right) \int_{\Omega} u_{t}(t) \Delta u(t) d x\|\nabla w(t)\|_{2}^{2} \leq C_{1}\|\nabla w(t)\|_{2}^{2}
$$

Ainda, observando que $M\left(a^{2}\right)-M\left(b^{2}\right) \leq M^{\prime}\left(\sup \left\{a^{2}, b^{2}\right\}\right)(|a|+|b|)|a-b|$, vemos que

$$
\begin{gathered}
\left(M\left(\|\nabla u(t)\|_{2}^{2}\right)-M\left(\|\nabla v(t)\|_{2}^{2}\right)\right) \int_{\Omega} \Delta v(t) w_{t}(t) d x \\
\leq C_{1}\|\nabla w(t)\|_{2}\left\|w_{t}(t)\right\|_{2} \\
\leq C_{1}\|\nabla w(t)\|_{2}^{\frac{r+2}{r+1}}+\frac{1}{4}\left\|w_{t}(t)\right\|_{r+2}^{r+2} .
\end{gathered}
$$


Aplicando adesigualdade de Hölder com $\frac{\rho}{2(\rho+1)}+\frac{1}{2(\rho+1)}+\frac{1}{2}=1$, inferimos que

$$
\begin{aligned}
\int_{\Omega}\left(f(u(t))-f(v(t)) w_{t}(t) d x\right. & \leq(\rho+1) \int_{\Omega} \sup \left\{|u|^{\rho},|v|^{\rho}\right\} w(t) w_{t}(t) d x \\
& \leq C_{1}\left(\|u(t)\|_{2(\rho+1)}^{\rho}+\|v(t)\|_{2(\rho+1)}^{\rho}\right)\|w(t)\|_{2(\rho+1)}\left\|w_{t}(t)\right\|_{2} \\
& \leq C_{1}\|\nabla w(t)\|_{2}\left\|w_{t}(t)\right\|_{r+2} \\
& \leq C_{1}\|\nabla w(t)\|_{2}^{\frac{r+2}{r+1}}+\frac{1}{4}\left\|w_{t}(t)\right\|_{r+2}^{r+2} .
\end{aligned}
$$

Por outro lado, a monotocidade de $g(s)=|s|^{r} s$ implica que

$$
\int_{\Omega}\left(g\left(u_{t}(t)\right)-g\left(v_{t}(t)\right)\right)\left(u_{t}(t)-v_{t}(t)\right) d x \geq\left\|w_{t}(t)\right\|_{r+2}^{r+2}
$$

Assim, substituindo (4.32)-(4.35) em (4.31) obtemos

$$
\begin{aligned}
\frac{d}{d t} E_{w}(t)+\left\|w_{t}(t)\right\|_{r+2}^{r+2} & \leq C_{1}\left(\|\nabla w(t)\|_{2}^{2}+\|\nabla w(t)\|_{2}^{\frac{r+2}{r+1}}\right) \\
& =C_{1}\|\nabla w(t)\|_{2}\left(\|\nabla w(t)\|_{2}+\|\nabla w(t)\|_{2}^{\frac{1}{r+1}}\right), \forall t \geq 0
\end{aligned}
$$

Note que, como $w=u-v$, a estimativa (4.8) implica que $\left\|\left(w(t), w_{t}(t)\right)\right\|_{E_{0}} \leq C_{1}, t \geq 0$. Assim, em (4.36), o último termo é também limitado. Então, integrando (4.36) de $t$ até $t+1$, obtemos

$$
\int_{t}^{t+1}\left\|w_{t}(s)\right\|_{r+2}^{r+2} d s \leq E_{w}(t)-E_{w}(t+1)+C_{D} \int_{t}^{t+1}\|\nabla w(s)\|_{2} d s \equiv D(t)^{2}
$$

onde $C_{D}>0$ é uma constante derivada de $C_{1}$. O funcional $D(t)$ irá desempenhar um papel semelhante àquele de $F(t)$ definido em (4.12).

Como em (4.16), (4.17) e (4.18), inferimos que

$$
\int_{t}^{t+1}\left\|w_{t}(s)\right\|_{2}^{2} d s \leq C_{1} D(t)^{\frac{4}{r+2}}
$$

e a existência de $t_{1} \in\left[t, t+\frac{1}{4}\right]$ e $t_{2} \in\left[t+\frac{3}{4}, t+1\right]$ tais que

$$
\left\|w_{t}\left(t_{i}\right)\right\|_{2}^{2} \leq C_{1} D(t)^{\frac{4}{r+2}}, \quad i=1,2
$$


o que implica que

$$
\left|\int_{\Omega} w_{t}\left(t_{2}\right) w\left(t_{2}\right) d x-\int_{\Omega} w_{t}\left(t_{1}\right) w\left(t_{1}\right) d x\right| \leq C_{1} D(t)^{\frac{4}{r+2}}+\frac{1}{8} \sup _{t \leq s \leq t+1} E_{w}(s) .
$$

Agora, multiplicando a equação em (4.29) por $w$ e integrando em $\Omega$, obtemos

$$
\begin{aligned}
\|\Delta w(t)\|_{2}^{2}+ & M\left(\|\nabla u(t)\|_{2}^{2}\right)\|\nabla w(t)\|_{2}^{2}+\int_{\Omega}(f(u(t))-f(v(t))) w(t) d x \\
= & \frac{d}{d t} \int_{\Omega} w_{t}(t) w(t) d x+\left\|w_{t}(t)\right\|_{2}^{2} \\
& +\left(M\left(\|\nabla u(t)\|_{2}^{2}\right)-M\left(\|\nabla v(t)\|_{2}^{2}\right)\right) \int_{\Omega} \Delta v(t) w(t) d x \\
& +\int_{\Omega}\left(g\left(u_{t}(t)\right)-g\left(v_{t}(t)\right)\right) w(t) d x .
\end{aligned}
$$

Integrando (4.40) de $t_{1}$ até $t_{2}$ e usando (4.38), (4.39) e a monotocidade de $f(s)=|s|^{\rho} s$, concluimos que

$$
\begin{aligned}
\int_{t_{1}}^{t_{2}}(\| \Delta w(s) & \left.\left\|_{2}^{2}+M\left(\|\nabla u(s)\|_{2}^{2}\right)\right\| \nabla w(s) \|_{2}^{2}\right) d s \\
\leq & C_{1} D(t)^{\frac{4}{r+2}}+\frac{1}{8} \sup _{t \leq s \leq t+1} E_{w}(s) \\
& +\int_{t_{1}}^{t_{2}}\left(M\left(\|\nabla u(s)\|_{2}^{2}\right)-M\left(\|\nabla v(s)\|_{2}^{2}\right)\right) \int_{\Omega} \Delta v(s) w(s) d x d s \\
& +\int_{t_{1}}^{t_{2}} \int_{\Omega}\left(g\left(u_{t}(s)\right)-g\left(v_{t}(s)\right)\right) w(s) d x d s .
\end{aligned}
$$

Com um procedimento semelhante ao usado em (4.34), $\operatorname{com} \frac{r}{r+2}+\frac{1}{r+2}+\frac{1}{r+2}=1$, inferimos que

$$
\int_{\Omega}\left(g\left(u_{t}(t)\right)-g\left(v_{t}(t)\right)\right) w(t) d x \leq C_{1}\left\|w_{t}(t)\right\|_{r+2}\|\Delta w(t)\|_{2}
$$

Combinando isto com (4.37) vemos

$$
\int_{t_{1}}^{t_{2}} \int_{\Omega}\left(g\left(u_{t}(s)\right)-g\left(v_{t}(s)\right)\right) w(s) d x d s \leq C_{1} D(t)^{\frac{4}{r+2}}+\frac{1}{8} \sup _{t \leq s \leq t+1} E_{w}(s) .
$$


Também, a estimativa (4.8) implica que

$$
\int_{t_{1}}^{t_{2}}\left(M\left(\|\nabla u(s)\|_{2}^{2}\right)-M\left(\|\nabla v(s)\|_{2}^{2}\right)\right) \int_{\Omega} \Delta v(s) w(s) d x d s \leq C_{1} \int_{t_{1}}^{t_{2}}\|\nabla w(s)\|_{2}^{2} d s
$$

Inserindo (4.42) e (4.43) em (4.41) obtemos

$$
\begin{aligned}
& \int_{t_{1}}^{t_{2}}\left(\|\Delta w(s)\|_{2}^{2}+M\left(\|\nabla u(s)\|_{2}^{2}\right)\|\nabla w(s)\|_{2}^{2}\right) d s \\
& \quad \leq C_{1} D(t)^{\frac{4}{r+2}}+\frac{1}{4} \sup _{t \leq s \leq t+1} E_{w}(s)+C_{1} \int_{t}^{t+1}\|\nabla w(s)\|_{2}^{2} d s .
\end{aligned}
$$

Então, da definição de $E_{w}(t),(4.38)$ e (4.44) resulta que

$$
\int_{t_{1}}^{t_{2}} E_{w}(s) d s \leq C_{1} D(t)^{\frac{4}{r+2}}+\frac{1}{4} \sup _{t \leq s \leq t+1} E_{w}(s)+C_{1} \int_{t}^{t+1}\|\nabla w(s)\|_{2}^{2} d s
$$

Agora, pelo Teorema do Valor Médio, existe $t^{*} \in\left[t_{1}, t_{2}\right]$ tal que

$$
E_{w}\left(t^{*}\right) \leq C_{1} D(t)^{\frac{4}{r+2}}+\frac{1}{2} \sup _{t \leq s \leq t+1} E_{w}(s)+C_{1} \int_{t}^{t+1}\|\nabla w(s)\|_{2}^{2} d s .
$$

Afirmamos que

$$
\sup _{t \leq s \leq t+1} E_{w}(s) \leq E_{w}\left(t^{*}\right)+D(t)^{2}+2 C_{D} \int_{t}^{t+1}\|\nabla w(s)\|_{2} d s .
$$

Com efeito, primeiramente notemos que de (4.37),

$$
E_{w}(t) \leq E_{w}(t+1)+D(t)^{2}
$$

Prosseguindo, seja $\tau \in[t, t+1]$ tal que $E_{w}(\tau)=\sup _{t \leq s \leq t+1} E_{w}(s)$. Então integrando (4.36) sobre $[t, \tau]$ e sobre $\left[t^{*}, t+1\right]$, temos 


$$
\begin{aligned}
E_{w}(\tau) & \leq E_{w}(t)+C_{D} \int_{t}^{t+1}\|\nabla w(s)\|_{2} d s \\
& \leq E_{w}(t+1)+D(t)^{2}+C_{D} \int_{t}^{t+1}\|\nabla w(s)\|_{2} d s \\
& \leq E_{w}\left(t^{*}\right)+D(t)^{2}+2 C_{D} \int_{t}^{t+1}\|\nabla w(s)\|_{2} d s
\end{aligned}
$$

e portanto (4.46) vale. Assim, inserindo (4.45) em (4.46) obtemos

$$
\begin{aligned}
\sup _{t \leq s \leq t+1} E_{w}(s) \leq & C_{1} D(t)^{\frac{2 r}{r+2}}\left(1+D(t)^{2-\frac{4}{r+2}}\right) \\
& +C_{1} \sup _{0 \leq \sigma \leq T} \int_{\sigma}^{\sigma+1}\|\nabla w(s)\|_{2}\left(1+\|\nabla w(s)\|_{2}\right) d s \\
\leq & C_{1} D(t)^{\frac{4}{r+2}}+C_{1} \sup _{0 \leq \sigma \leq T} \int_{\sigma}^{\sigma+1}\|\nabla w(s)\|_{2} d s,
\end{aligned}
$$

uma vez que os termos entre parênteses são limitados. Portanto vemos

$$
\begin{aligned}
\sup _{t \leq s \leq t+1} E_{w}(s)^{\frac{r+2}{2}} & \leq C_{1} D(t)^{2}+C_{1}\left(\sup _{0 \leq \sigma \leq T} \int_{\sigma}^{\sigma+1}\|\nabla w(s)\|_{2} d s\right)^{\frac{r+2}{2}} \\
& \leq C_{1} D(t)^{2}+C_{2} \sup _{0 \leq \sigma \leq T} \int_{\sigma}^{\sigma+1}\|\nabla w(s)\|_{2} d s
\end{aligned}
$$

onde

$$
C_{2}=C_{1}\left(\sup _{0 \leq \sigma \leq T} \int_{\sigma}^{\sigma+1}\|\nabla w(s)\|_{2} d s\right)^{\frac{r}{2}}
$$

é uma constante que depende de $T$. Portanto, combinando (4.37) com (4.47), segue que

$$
\sup _{t \leq s \leq t+1} E_{w}(s)^{1+\frac{r}{2}} \leq C_{1}\left(E_{w}(t)-E_{w}(t+1)\right)+\left(C_{D}+C_{2}\right) \sup _{0 \leq \sigma \leq T} \int_{\sigma}^{\sigma+1}\|\nabla w(s)\|_{2} d s .
$$


Então, aplicando o Lema de Nakao obtemos

$$
\begin{aligned}
E_{w}(t) \leq & \left(C_{1}^{-1} \frac{r}{2}(t-1)^{+}+\left(\sup _{0 \leq s \leq 1} E_{w}(s)\right)^{-\frac{r}{2}}\right)^{-\frac{2}{r}} \\
& +\left(\left(C_{D}+C_{2}\right) \sup _{0 \leq \sigma \leq T} \int_{\sigma}^{\sigma+1}\|\nabla w(s)\|_{2} d s\right)^{\frac{2}{r+2}}, \quad 0 \leq t \leq T .
\end{aligned}
$$

Isto completa a prova do Lema 4.2.2.

Prosseguindo, usaremos o Lema 4.2.2 para provar a compacidade assintótica do semigrupo correspondente ao problema (4.1) em $E_{0}=H_{0}^{2}(\Omega) \times L^{2}(\Omega)$.

Lema 4.2.3 Sobre as hipóteses do Teorema 4.2.1, o semigrupo $S(t)$ é compactamente assintótico em $E_{0}$.

\section{Demonstração:}

Sejam $\left(u_{0}, u_{1}\right),\left(v_{0}, v_{1}\right)$ dados num conjunto limitado $B \subset E_{0}$. Sejam $u_{n}(t)=S(t)\left(u_{0}, u_{1}\right)$ e $v_{n}(t)=S(t)\left(v_{0}, v_{1}\right)$ as correspondentes soluções do problema (4.1). Então sabemos que

$$
u, v \in C^{0}\left([0, \infty) ; H_{0}^{2}(\Omega)\right) \cap C^{1}\left([0, \infty) ; L^{2}(\Omega)\right),
$$

conforme Teorema 4.1.1.

Pelo lemma anterior, fixado $T>0$, differença $w(t)=u(t)-v(t)$ satisfaz

$$
\begin{aligned}
& \left\|u_{t}(t)-v_{t}(t)\right\|_{2}^{2}+\|\Delta u(t)-\Delta v(t)\|_{2}^{2} \\
& \quad \leq C_{B}(T-1)^{-\frac{2}{r}}+C_{T}\left(\sup _{0 \leq \sigma \leq T} \int_{0}^{\sigma+1}\|\nabla u(s)-\nabla v(s)\|_{2} d s\right)^{\frac{2}{r+2}}
\end{aligned}
$$

Então, dado $\varepsilon>0$, podemos escolher $T>0$ suficientemente grande de forma que

$$
\left\|S(T)\left(u_{0}, u_{1}\right)-S(T)\left(v_{0}, v_{1}\right)\right\|_{E_{0}} \leq \varepsilon+C_{T}\left(\sup _{0 \leq \sigma \leq T} \int_{0}^{\sigma+1}\|\nabla u(s)-\nabla v(s)\|_{2} d s\right)^{\frac{1}{r+2}}
$$

Seja $B_{0}$ o conjunto absorvente obtido no Lemma 4.2.1. Definimos $\phi_{T}: B_{0} \times B_{0} \rightarrow \mathbb{R}$ como 
sendo o lado direito de (4.48), isto é,

$$
\phi_{T}\left(\left(u_{0}, u_{1}\right),\left(v_{0}, v_{1}\right)\right)=C_{T}\left(\sup _{0 \leq \sigma \leq T} \int_{0}^{\sigma+1}\|\nabla u(s)-\nabla v(s)\|_{2} d s\right)^{\frac{1}{r+2}}
$$

onde $u, v$ são soluções do problema (4.1) correspondente aos dados $\left(u_{0}, u_{1}\right),\left(v_{0}, v_{1}\right)$. Para aplicar o Lemma 1.3.2, falta mostrar que para qualquer sequência $\left\{\left(u_{0}^{n}, u_{1}^{n}\right)\right\}$ de $B_{0} \subset E_{0}$, existe uma subsequência $\left\{\left(u_{0}^{n_{k}}, u_{1}^{n_{k}}\right)\right\}$ tal que

$$
\lim _{k \rightarrow \infty} \lim _{l \rightarrow \infty} \phi_{T}\left(\left(u_{0}^{n_{k}}, u_{1}^{n_{k}}\right),\left(u_{0}^{n_{l}}, u_{1}^{n_{l}}\right)\right)=0 .
$$

Observamos que como $B_{0}$ é limitado e invariante sob $\{S(t)\}_{t \geq 0}$, as soluções $\left\{\left(u^{n}(t), u_{t}^{n}(t)\right)\right\}$ são uniformemente limitadas em $E_{0}$. Portanto

$$
u^{n} \text { é limitado em } C\left([0, \infty), H_{0}^{2}(\Omega)\right) \cap C^{1}\left([0, \infty) ; L^{2}(\Omega)\right) .
$$

Por outro lado, a imersão compacta de $H_{0}^{2}(\Omega)$ em $H_{0}^{1}(\Omega)$ implica que a imersão

$$
C\left([0, \infty), H_{0}^{2}(\Omega)\right) \cap C^{1}\left([0, \infty) ; L^{2}(\Omega)\right) \hookrightarrow C\left([0, T] ; H_{0}^{1}(\Omega)\right)
$$

é compacta para cada $T>0$. (Simon [46]).

Assim $u^{n}$ possui uma subsequência $u^{n_{k}}$ que converge forte em $C\left([0, T] ; H_{0}^{1}(\Omega)\right)$, e portanto temos que o limite (4.49) é verdadeiro. 


\section{Referências Bibliográficas}

[1] A. Arosio, A geometrical nonlinear correction to the Timoshenko beam equation, Nonlinear Analysis. 47 (2001) 729-740.

[2] A. Arosio \& S. Panizzi, On the well-posedness of the Kirchhoff string, Trans. Amer. Math. Soc. 348 (1996) 305-330.

[3] A. V. Babin \& M. I. Visik, Atractors of Evolution Equatios, Nauka, Moscow, 1989; English translation, North-Holland, 1992.

[4] J. M. Ball, Initial-boundary value problems for an extensible beam, J. Math. Anal. Appl. 42 (1973) 61-90.

[5] J. M. Ball, Stability theory for an extensible beam, J. Differential Equations 14 (1973) $399-418$.

[6] J. M. Ball, Strongly continuous semigroups, weak solutions and the variation of constants formula, Proc. Amer, Math. Soc. 63 (1997) 370-373.

[7] J. M. Ball, Global attractor for damped semilinear wave equations, Discrete and Continuous Dynamical Systems 10 (2004) 31-52.

[8] H. M. Berger, A new approach to the analysis of large deflections of plates, Journal of Applied Mechanics 22 (1955) 465-472.

[9] S. Bernstein, Sur une classe d'équations fonctionelles aux derivées partielles, Isv. SSSR, Serie Math. 4 (1940) 17-26. 
[10] A. C. Biazutti \& H. R. Crippa, Global attractor and inertial set for the beam equation, Applicable Analysis 55 (1994) 61-78.

[11] P. Biler, Remark on the decay for damped string and beam equations, Nonlinear Analysis 10 (1986) 839-842.

[12] H. Brézis, Analyse Fonctionnelle, Theorie et Applications, Masson, Paris, 1983.

[13] E. H. Brito, Decay estimates for the generalized damped extensible string and beam equations, Nonlinear Analysis. 8 (1984) 1489-1496.

[14] M. M. Cavalcanti, V. N. Cavalcanti \& J. A. Soriano, Global existence and asymptotic stability for the nonlinear and generalized damped extensible plate equation, Communications in Contemporary Mathematics. 6 (2004) 705-731.

[15] R. C. Charão, E. Bisognin, V. Bisognin \& A. F. Pazoto, Asymptotic behavior of a BernoulliEuler type equation with nonlinear localized damping, Progress in Nonlinear Differential Equations and Their Applications, 66 (2005) 67-91.

[16] I. Chueshov \& I. Lasiecka, Long-Time Behavior of Second Order Evolution Equations with Nonlinear Damping. Mem. Amer. Math. Soc. 195, Number 912, Providence, 2008.

[17] A. T. Cousin, C. L. Frota \& N. A. Lar'kin, Global solvability and decay of the energy for the nonhomogeneous Kirchhoff equation, Differential and Integral Equations 15 (2002) 1219-1236.

[18] R. W. Dickey, Infinite system of nonlinear oscillation equations related to the string, Proc. of the A.M.S. 23 (1969) 459-468.

[19] R. W. Dickey, Free vibrations and dynamic bucking of the extensible beam, J. Math. Anal. Appl. 29 (1970) 443-454.

[20] R. W. Dickey, A quasilinear evolution equation and the method of Galerkin, Proc. of the A.M.S. 37(1) (1973) 149-156.

[21] A. Eden \& A. J. Milani, Exponential attractor for extensible beam equations, Nonlinearity. 6 (1993) 457 - 479. 
[22] A. Eden, C. Foias, B. Nicolaenko \& R. Temam, Exponential Attractors for Dissipative Evolution Equations, Wiley-Masson, Paris, 1994.

[23] J. G. Eisley, Nonlinear vibrations of beams and rectangular plates, Z. Angew Math. Phys. 15 (1964) 167-175.

[24] J. K. Hale, Asymptotic Behavior of Dissipative Systems, Amer. Math. Soc., Providence, 1988.

[25] A. Kh. Khanmamedov, Global attractors for the plate equation with a localized damping and critical exponent in an unbounded domain, J. Differential Equation 225 (2006), 528548 .

[26] G. Kirchhoff, Vorlessunger über Mathematiche Physik, Mechanik, Teubner, Leipzig, 1876.

[27] S. Kouémou Patcheu, On a global solution and asymptotic behaviour for the generalized damped extensible beam equation, J. Differential Equations 135 (1997) 299-314.

[28] J. L. Lions \& E. Magenes, Problèmes aux Limites Non Homogènes et Applications, Dunod, Paris, 1968.

[29] J. L. Lions, Quelques Méthodes de Résolution des Problèmes Aux Limites Non Linèaires, Dunod, Paris, 1969.

[30] J. L. Lions, On some questions in boundary value problems of mathematical physics, in: G.M. de La Penha and L.A. Medeiros (Eds.), Contemporary Developments in Continuum Mechanics and Partial Differential Equations, North-Holland Mathematics Studies 30, North-Holland, Amsterdam, (1978) 284-346.

[31] T. F. Ma, Boundary stabilization for a nonlinear beam on elastic bearings, Math. Meth. Appl. Science 24 (2001) 583-594.

[32] T. F. Ma, Remarks on an elliptic equation of Kirchhoff type, Nonlinear Analysis 63 (2005) 1967-1977.

[33] T. F. Ma \& V. Narciso, Global attractor for a model of extensible beam with nonlinear damping and source terms, Preprint (2010). 
[34] L. A. Medeiros, On a new class of nonlinear wave equations, J. Math. Anal. and Appl. 69 (1979) 252-262.

[35] L. A. Medeiros, Exact controllability for a Timoshenko model of vibrations of beams, Adv. in Math. Sci. and Appl., Tokyo, 2(1) (1993) 47 -61.

[36] J. E. Muñoz Rivera, Global solution and regularizing properties on a class of nonlinear evolution equation, J. Differential Equations 128 (1996) 103-124.

[37] M. Nakao, Global attractors for wave equations with nonlinear dissipative terms, J. Differential Equations 227 (2006) 204 - 229.

[38] M. Nakao \& T. Narazaki, Existence and decay of solutions of some nonlinear wave equations in noncylindrical domains, Math. Rep. XI 2 (1978) 117-125.

[39] V. Narciso, Global attractor for a plate equation with a nonlocal dissipation, Preprint (2010).

[40] A. Nayfeh \& D. T. Mook, Nonlinear Oscilations, John Willey 1979.

[41] A. F. Pazoto \& P. G. Menzala, Uniform rates of decay of a nonlinear beam model with thermal effects and nonlinear boundary dissipation, Funkcialaj Ekvacioj 43 (2000) 339360.

[42] A. Pazy, Semigroups of Linear Operators and Applications to Partial Differential Equation, Appied Mathematical Sciences, 44, Springer - Verlag, New York 1983.

[43] G. Perla Menzala, A. F. Pazoto \& E. Zuazua, Stabilization of Berger-Timoshenko's equation as limit of the uniform stabilization of the von Kármán system of beams and plates, M2AN Math. Model. Numer. Anal. 36 (2002) 657-691.

[44] S. L. Pohozhaev, On a class of quasilinear hyperbolic equations, Math. Sb. 25 (1975) 145-158.

[45] D. Ševčovič, Existence and limiting behaviour for damped nonlinear evolution equations with nonlocal terms, Comment. Math. Univ. Carolin. 31(2) (1990) 283-293.

[46] J. Simon, Compact sets in the space $L^{p}(0, T ; B)$, Ann. Mat. Pura Appl. 146 (1987), 65-96. 
[47] W. A. Straus, On weak solutions of semilinear hyperbolic equations, Anais Acad. Bras. Ciências, 42 (1970) 645-651.

[48] R. Temam, Infinite Dimensional Dynamic System in Mechanics and Physics, Springer Verlag, New York, 1988.

[49] S. Woinowsky-Krieger, The effect of axial force on the vibration of hinged bars, Journal of Applied Mechanics 17 (1950) 35-36.

[50] K. Yosida, Functional Analysis, 3rd ed., Springer, Berlin 1971.

[51] Songmu Zheng, Nonlinear Evolution Equations, Chapman \& Hall/CRC, Boca Raton, 2004. 